\title{
Combinatorial modulus, the combinatorial Loewner property, and Coxeter groups
}

\author{
Marc Bourdon and Bruce Kleiner
}

\begin{abstract}
We study combinatorial modulus on self-similar metric spaces. We give new examples of hyperbolic groups whose boundaries satisfy a combinatorial version of the Loewner property, and prove Cannon's conjecture for Coxeter groups. We also establish some connections with $\ell_{p}$-cohomology.
\end{abstract}

Mathematics Subject Classification (2010). 20F65, 20F67, 30L10.

Keywords. Geometric group theory, hyperbolic groups and nonpositively curved groups, quasiconformal mappings in metric spaces.

\section{Contents}

1 Introduction . . . . . . . . . . . . . . . . . . . . . . . 39

2 Combinatorial modulus . . . . . . . . . . . . . . . . . . . 46

3 Self-similarity ............................. 57

4 The combinatorial Loewner property for the standard Sierpinski carpet and Menger curve . . . . . . . . . . . . . . . . . . . . . . . 64

5 Hyperbolic Coxeter groups . . . . . . . . . . . . . . . . . . . . 70

6 Combinatorial modulus and Coxeter groups . . . . . . . . . . . . . . . 78

7 Cannon Coxeter groups . . . . . . . . . . . . . . . . . . . . . . . . 84

8 The combinatorial Loewner property for Coxeter groups . . . . . . . . . . . . . . 87

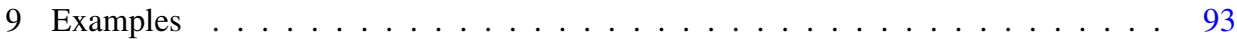

$10 \ell_{p}$-equivalence relations $\ldots \ldots \ldots \ldots \ldots \ldots$

References . . . . . . . . . . . . . . . . . . . . . . . . . . 104

\section{Introduction}

1.1. Overview. Every hyperbolic group $\Gamma$ has a canonical action on its boundary at infinity $\partial \Gamma$; with respect to any visual metric on $\partial \Gamma$, this action is by uniformly quasi-Möbius homeomorphisms. This structure has a central role in the proofs of Mostow's rigidity theorem and numerous other results in the same vein, which are 
based on the analytic theory of quasiconformal homeomorphisms of the boundary (see the survey papers [GP91], [BP02], [Kle06] and their references). With the aim of extending these rigidity results to a larger class of hyperbolic groups, one may hope to apply the work of J. Heinonen and P. Koskela [HK98] and subsequent authors (e.g. [Che99], [Tys98], [HKST01], [KZ08]), which has generalized much of the classical quasiconformal theory to the setting of Loewner spaces ${ }^{1}$, a certain class of metric measure spaces [HK98]. Unfortunately, among the currently known examples of Loewner spaces, the only ones which arise as boundaries of hyperbolic groups are the boundaries of rank-one symmetric spaces, and Fuchsian buildings [HK98], [BP00]. One of the goals of this paper is to take a step toward improving this situation, by finding infinitely many new examples of hyperbolic groups whose boundaries satisfy the combinatorial Loewner property, a combinatorial variant of the Loewner property which is conjecturally equivalent to the property of being quasi-Möbius homeomorphic to a Loewner space. In addition to this, using similar techniques, we prove the Cannon conjecture for hyperbolic Coxeter groups, show that the $\ell_{p^{-}}$ equivalence relation studied in [Gro93], [Ele97], [Bou04] has a particularly simple form in the case of hyperbolic Coxeter groups, and prove that the standard square Sierpinski carpet in the plane and the standard cubical Menger curve in $\mathbb{R}^{3}$ satisfy the combinatorial Loewner property.

1.2. Statement of results. We now present some of the ideas of the paper, illustrating them with non-technical statements. More general results, as well as detailed discussion justifying the statements made here, may be found in the body of the paper.

Combinatorial modulus. Let $Z$ be a compact metric space. For every $k \in \mathbb{N}$, let $G_{k}$ be the incidence graph of a ball cover $\left\{B\left(x_{i}, 2^{-k}\right)\right\}_{i \in I}$, where $\left\{x_{i}\right\}_{i \in I} \subset Z$ is a maximal $2^{-k}$-separated subset. Given $p \geq 1$ and a curve family $\mathscr{F}$ in $Z$, we denote by $\operatorname{Mod}_{p}\left(\mathscr{F}, G_{k}\right)$ the $G_{k}$-combinatorial $p$-modulus of $\mathcal{F}$ (see Subsection 2.1 for the definition); also for any pair of subsets $A, B \subset Z$, we let $\operatorname{Mod}_{p}\left(A, B, G_{k}\right)=$ $\operatorname{Mod}_{p}\left(\mathcal{F}, G_{k}\right)$ where $\mathcal{F}$ is the collection of paths joining $A$ and $B$.

In our study of combinatorial modulus, we will assume that $Z$ is approximately self-similar (see Definition 3.1). Examples of approximately self-similar metric spaces include many classical fractals such as the square Sierpinski carpet or the cubical Menger curve, boundaries of hyperbolic groups equipped with their visual metrics, metric spaces associated with finite subdivision rules, and metric 2 -spheres arising from expanding Thurston map.

One of our principal goals is to find criteria for the combinatorial Loewner property (CLP). Roughly speaking, a doubling space $Z$ satisfies the CLP if there is a $p \in(1, \infty)$ such that for any pair $A, B \subset Z$ of disjoint nontrivial continua, the $p$-modulus

\footnotetext{
${ }^{1}$ We will use the shorthand Loewner space for a compact metric space that is Ahlfors $p$-regular and $p$-Loewner for some $p>1$, in the sense of [HK98].
} 
$\operatorname{Mod}_{p}\left(A, B, G_{k}\right)$ is controlled by the relative distance

$$
\Delta(A, B)=\frac{\operatorname{dist}(A, B)}{\min \{\operatorname{diam} A, \operatorname{diam} B\}} ;
$$

see Section 2.2 for the definition. As indicated in the overview, our interest in the CLP stems from the fact that a Loewner space satisfies the CLP, and that the converse is conjecturally true for compact approximately self-similar spaces. Thus - modulo the conjecture - this paper would provide new examples of hyperbolic groups to which the recently developed quasiconformal theory would be applicable. Should the conjecture turn out to be false, the CLP would be of independent interest, since it shares many of the features of the Loewner property, e.g. quasi-Möbius invariance; see Section 2.2 for more discussion.

Coxeter group boundaries satisfying the CLP. Recall that a group $\Gamma$ is a Coxeter group if it admits a presentation of the form

$$
\left.\Gamma=\left\langle s_{i} \in S\right| s_{i}^{2}=1,\left(s_{i} s_{j}\right)^{m_{i j}}=1 \text { for } i \neq j\right\rangle,
$$

with $|S|<+\infty$, and with $m_{i j} \in\{2,3, \ldots,+\infty\}$. A subgroup is special if it is generated by a subset of the generating set $S$. A subgroup is parabolic if it is conjugate to a special subgroup. Now suppose in addition that $\Gamma$ is hyperbolic, and $\partial \Gamma$ is its boundary at infinity. Then a non-empty limit set $\partial P \subset \partial \Gamma$ of a parabolic subgroup $P \leqq \Gamma$ is called a parabolic limit set.

In Theorem 8.6 we give a sufficient condition (of combinatorial flavour) for the boundary of a hyperbolic Coxeter group to satisfy the CLP. A special case is the following result, which shows that the CLP holds when the parabolic limit sets form a combinatorially simple collection of subsets.

Theorem 1.1 (Corollary 8.7). Let $\Gamma$ be a hyperbolic Coxeter group whose boundary is connected and such that $\operatorname{Confdim}(\partial \Gamma)>1$. Assume that for every proper, connected, parabolic limit set $\partial P \subset \partial \Gamma$, one has

$$
\operatorname{Confdim}(\partial P)<\operatorname{Confdim}(\partial \Gamma) .
$$

Suppose furthermore that for every pair $\partial P, \partial Q$ of distinct, proper, connected, parabolic limit sets, the subset $\partial P \cap \partial Q$ is totally disconnected or empty. Then ӘГ satisfies the CLP.

In the statement $\operatorname{Confdim}(Z)$ denotes the Ahlfors regular conformal dimension of $Z$ i.e. the infimum of Hausdorff dimensions of Ahlfors regular metric spaces quasi-Möbius homeomorphic to $Z$ (see [MT10] for more details about conformal dimension).

To illustrate Theorem 1.1 with some simple examples, consider a Coxeter group $\Gamma$ with a Coxeter presentation

$$
\left.\left\langle s_{1}, \ldots, s_{4}\right| s_{i}^{2}=1,\left(s_{i} s_{j}\right)^{m_{i j}}=1 \text { for } i \neq j\right\rangle,
$$


where the order $m_{i j}$ is finite for all $i \neq j$, and for every $j \in\{1, \ldots, 4\}$ one has $\sum_{i \neq j} \frac{1}{m_{i j}}<1$. For these examples, the proper connected parabolic limit sets are circles, and hence have conformal dimension 1 , while $\partial \Gamma$ is homeomorphic to the Sierpinski carpet and therefore has conformal dimension $>1$ by a result of J. Mackay [Mac10]. Theorem 1.1 therefore applies, and $\partial \Gamma$ has the CLP.

Applying similar techniques in a simplified setting, we prove:

Theorem 1.2 (Theorem 4.1). The square Sierpinski carpet and the cubical Menger curve satisfy the CLP.

The Cannon conjecture for Coxeter groups. We obtain a proof of Cannon's conjecture in the special case of Coxeter groups:

Theorem 1.3 (Theorem 7.1). Let $\Gamma$ be a hyperbolic Coxeter group whose boundary is homeomorphic to the 2-sphere. Then there is a properly discontinuous, cocompact, and isometric action of $\Gamma$ on $\mathbb{H}^{3}$.

This result was essentially known (see the discussion at the end of Section 7). Our view is that the principal value of the proof is that it illustrates the feasibility of the asymptotic approach (using the ideal boundary and modulus estimates), and it may suggest ideas for attacking the general case. It also gives a new proof of the Andreev's theorem on realizability of polyhedra in $\mathbb{H}^{3}$, in the case when the prescribed dihedral angles are submultiples of $\pi$.

$\ell_{p}$-equivalence relations. Let $\Gamma$ be a hyperbolic group and let $p \geq 1$. The first $\ell_{p^{-}}$ cohomology group of $\Gamma$ induces on $\partial \Gamma$ an equivalence relation - the $\ell_{p}$-equivalence relation - which is invariant under quasi-isometries of $\Gamma$ [Gro93], [Ele97], [BP03], [Bou04]. A natural problem is to determine its cosets. The existence of a non trivial coset, i.e. a coset different from a point and from the whole space, was shown to be an obstruction to the Loewner property in [BP03]. Inspired by this, we prove the analogous statement for the CLP in Corollary 10.5. Moreover, in consequence of some of our previous results, we get:

Corollary 1.4 (Corollaries 10.3 and 10.4). Assume $\Gamma$ is a hyperbolic Coxeter group, let $p \geq 1$, and denote by $\sim_{p}$ the $\ell_{p}$-equivalence relation on $\partial \Gamma$. Then:

(1) Each coset of $\sim_{p}$ is either a point or a connected parabolic limit set.

(2) If $\partial \Gamma$ is connected, and $\sim_{p}$ admits a coset different from a point and the whole set $\partial \Gamma$, then $\sim_{p}$ admits a coset $F$ with $\operatorname{Confdim}(F)=\operatorname{Confdim}(\partial \Gamma)$.

Beyond the CLP. At present, our understanding of Coxeter groups is still quite limited. We have only been able to show that certain very special groups have boundaries which satisfy the CLP. While this is consistent with our expectation that the CLP should be a highly non-generic property, we only have a few examples which 
are known not to have the CLP, apart from groups whose boundaries could not have the CLP for topological reasons, see Proposition 2.5 and the remark at the end of Section 10.

It would be desirable to have effective criteria for showing that a group boundary does not have the CLP, as well as new examples of such groups. In addition, when a group boundary does not have the CLP, we expect that alternative structure will be present instead, such as a quasi-Möbius invariant equivalence relation.

1.3. Discussion of the proofs. We now give an indication of the ideas that go into some of the proofs.

Combinatorial modulus on approximately self-similar spaces. Let $Z$ be a compact, approximately self-similar space. For $d_{0}>0$, let $\mathscr{F}_{0}$ be the family of curves $\gamma \subset Z$ with $\operatorname{diam}(\gamma) \geq d_{0}$. The approximate self-similarity of $Z$ allows one to compare moduli of curve families at different locations and scales with the modulus of $\widetilde{F}_{0}$, and this observation leads readily to a submultiplicativity relation between combinatorial moduli at different scales:

Proposition 1.5 (Proposition 3.6). Let $Z$ be an arcwise connected approximately self-similar metric space. Let $p \geq 1$ and set $M_{k}:=\operatorname{Mod}_{p}\left(\mathcal{F}_{0}, G_{k}\right)$. Then, for $d_{0}$ sufficiently small, there exists a constant $C>0$ such that for every pair of integers $k$, $\ell$ one has

$$
M_{k+\ell} \leq C \cdot M_{k} \cdot M_{\ell}
$$

In addition when $p$ belongs to a compact subset of $[1,+\infty)$ the constant $C$ may be chosen independent of $p$.

Iterating (1.1) yields $M_{n k} \leq C^{n-1} M_{k}^{n}$, which implies that $\lim _{j \rightarrow \infty} M_{j}=0$ if $M_{k}<C^{-1}$ for some $k$. Therefore if we define the critical exponent to be

$$
Q_{M}=\inf \left\{p \in[1, \infty) ; \lim _{k \rightarrow \infty} M_{k}=0\right\}
$$

then $M_{k}=\operatorname{Mod}_{Q_{M}}\left(\mathcal{F}_{0}, G_{k}\right) \geq C^{-1}$ for all $k$. In fact, $Q_{M}$ is the Ahlfors regular conformal dimension of $Z,[\mathrm{KeK}]$.

When $Z$ is the standard Sierpinski carpet constructed from the unit square, one can exploit the reflectional symmetry to get additional control on the modulus. Using it, one shows that for any nonconstant curve $\eta:[0,1] \rightarrow Z$ and any $\epsilon>0$, if $u_{\epsilon}(\eta)$ denotes the $\epsilon$ neighborhood of $\eta$ in the $C^{0}$ topology, then the $p$-modulus $\operatorname{Mod}_{p}\left(U_{\epsilon}(\eta), G_{k}\right)$ is uniformly comparable to $M_{k}$, independent of $k$. In other words, the modulus of the curves near an arbitrary curve is comparable to the modulus of all curves. From this, and using the planarity of the carpet, one can prove a supermultiplicativity inequality as well:

$$
M_{k+\ell} \geq C^{\prime} \cdot M_{k} \cdot M_{\ell}
$$


where $C^{\prime} \in(0, \infty)$ may be chosen in terms of an upper bound on $p$. Reasoning as above, it follows that if $M_{k}>C^{\prime-1}$ for any $k$, then $\lim _{k \rightarrow \infty} M_{k}=\infty$; this implies that at the critical exponent $Q_{M}$, the sequence $\left\{M_{k}\right\}$ is bounded away from zero and infinity. From this, one concludes that the statement of the CLP holds for pairs of balls. By imitating an argument from [BK05a], one shows that the CLP holds provided it holds for pairs of balls (Proposition 2.9), and therefore the Sierpinski carpet satisfies the CLP (see Theorem 4.1).

In the case of the Menger curve, instead of (1.2), one obtains an estimate for $M_{k}$ in terms of the moduli $\left\{M_{j}\right\}_{j<k}$, which is sufficient to verify the CLP.

Dynamics of curves and crossing. Our strategy for understanding the boundary of a hyperbolic Coxeter group $\Gamma$ is inspired by the analysis of the Sierpinski carpet, although the story is more complicated.

For the purpose of this paper, much of the dynamics of the $\Gamma$-action on $\partial \Gamma$ is encoded in the parabolic limit sets. For example we get:

Theorem 1.6 (Corollary 5.8). Consider a $\Gamma$-invariant equivalence relation on $\partial \Gamma$ whose cosets are connected. Then:

- The closure of each coset is either a point or a parabolic limit set.

- If a nontrivial coset $F$ is path-connected, and $P$ is the parabolic subgroup with $\bar{F}=\partial P$, then for every $\epsilon>0$ and every path $\eta:[0,1] \rightarrow \partial P$, there is a path $\eta^{\prime}:[0,1] \rightarrow F$ such that

$$
d\left(\eta, \eta^{\prime}\right)=\max _{t \in[0,1]} d\left(\eta(t), \eta^{\prime}(t)\right)<\epsilon
$$

As an illustration, let $\gamma$ be a nontrivial curve in $\partial \Gamma$, and let $\sim$ be the smallest equivalence relation on $\partial \Gamma$ such that for every $g \in \Gamma$, the curve $g \gamma$ lies in a single coset; in other words, two points $x, y \in \partial \Gamma$ lie in the same coset if there is a finite chain $g_{1} \gamma, \ldots, g_{k} \gamma$ joining $x$ to $y$. Thanks to the previous theorem, the coset closures are either points or parabolic limit sets. In particular, if $\gamma$ is not contained in any proper parabolic limit set, then any path $\eta:[0,1] \rightarrow \partial \Gamma$ is a uniform limit of paths lying in the coset of $\gamma$.

A key ingredient in the analysis of the combinatorial modulus on $\partial \Gamma$ is a quantitative version of this phenomenon, which is established in Proposition 5.12.

The proof of the Cannon conjecture for Coxeter groups. By [Sul81], if $\Gamma$ is a hyperbolic group and $\partial \Gamma$ is quasi-Möbius homeomorphic to the Euclidean 2-sphere, then $\Gamma$ admits a properly discontinuous, cocompact, isometric action on $\mathbb{H}^{3}$. Also, as a consequence of the uniformization theorem established in [BK02], we obtain:

Corollary 1.7 (Corollary 3.5). Suppose $Z$ is an approximately self-similar metric space homeomorphic to the 2-sphere. Assume that for $d_{0}>0$ small enough, there 
exists a constant $C=C\left(d_{0}\right) \geq 1$ such that for every $k \in \mathbb{N}$ one has

$$
\operatorname{Mod}_{2}\left(\mathcal{F}_{0}, G_{k}\right) \leq C \text {. }
$$

Then $Z$ is quasi-Möbius homeomorphic to the Euclidean 2-sphere.

Thus, we are reduced to verifying the hypotheses of the above corollary when $\Gamma$ is a Coxeter group. We note that an alternate reduction to the same assertion can be deduced using [CFP99].

One of the main results of the paper is the existence of a finite number of "elementary curves families", whose moduli govern the modulus of every (thick enough) curve family in $\partial \Gamma$ (see Theorem 6.1 and Corollary 6.2). Each elementary curve family is associated to a conjugacy class of an infinite parabolic subgroup.

In consequence, to obtain the bound (1.3), it is enough to establish that every connected parabolic limit set $\partial P$ enjoys the following property: there exists a non constant continuous curve $\eta \subset \partial P$ such that letting $U_{\epsilon}(\eta)$ be the $\epsilon$-neighborhood of $\eta$ in the $C^{0}$-topology, the modulus $\operatorname{Mod}_{2}\left(U_{\epsilon}(\eta), G_{k}\right)$ is bounded independently of $k$, for $\epsilon>0$ small enough.

To do so, two cases are distinguished: either $\partial P$ is a circular limit set i.e. it is homeomorphic to the circle, or it is not.

In the second case one can find two crossing curves $\eta_{1}, \eta_{2} \subset \partial P$. Since $\partial \Gamma$ is a planar set, one gets that $\min _{i=1,2} \operatorname{Mod}_{2}\left(U_{\epsilon}\left(\eta_{i}\right), G_{k}\right)$ is bounded independently of $k$, for $\epsilon$ small enough. Note that crossing type arguments in relation with the combinatorial 2-modulus, appear frequently in the papers [Can94], [CS98] and [CFP99] (not to mention the whole body of literature on 2-dimensional quasiconformal geometry).

Let $r>0$, and denote by $\mathscr{F}_{1}$ the subfamily of $\widetilde{F}_{0}$ consisting of the curves $\gamma \in \mathcal{F}_{0}$ which do not belong to the $r$-neighborhood $N_{r}(\partial P)$ of any circular limit set $\partial P$. At this stage one knows that for $r$ small enough, $\operatorname{Mod}_{2}\left(\mathscr{F}_{1}, G_{k}\right)$ is bounded independently of $k$. To bound the modulus of $\widetilde{F}_{0} \backslash \mathcal{F}_{1}$, we proceed as follows. Consider a curve $\gamma \in \mathscr{F}_{0}$ contained in $N_{r}(\partial P)$, where $\partial P$ is a circular parabolic limit set. The idea is to break $\gamma$ into pieces $\gamma_{1}, \ldots, \gamma_{i}$ such that for each $j \in\{1, \ldots, i\}$, the maximal distance $\max \left\{d(x, \partial P) ; x \in \gamma_{j}\right\}$ is comparable to $\operatorname{diam}\left(\gamma_{j}\right)$. Then for each $j$, applying a suitable group element $g \in \Gamma$, we can arrange that both $g \gamma_{j}$ and $g(\partial P)$ have roughly unit diameter. Since $g \gamma_{j}$ lies close to $g(\partial P)$, but not too close, it cannot lie very close to a circular limit set; it follows that $g \gamma_{j}$ belongs to a curve family with controlled modulus. We then apply $g^{-1}$ to the corresponding admissible function, and renormalize it suitably; by summing the collection of functions which arise in this fashion from all such configurations, we arrive at an admissible function for all such curves $\gamma$. The fact that the conformal dimension of $S^{1}$ is strictly less than 2 allows us to bound the 2-mass of this admissible function, and this yields the desired bound (1.3).

We note that in the body of the paper, the argument in the preceding paragraph appears in Theorem 6.3, where it is formulated in greater generality. It is also used in the proofs of Theorem 8.6 and Corollary 10.4. 
1.4. Organization of the paper. Combinatorial modulus, combinatorial Loewner property, and approximately self-similar metric spaces are presented in Sections 2 and 3. In Section 4 the combinatorial Loewner property is established for the square Sierpinski carpet and the cubical Menger curve. Section 5 contains preliminary results about various dynamical aspects of the action of a hyperbolic Coxeter group on its boundary. Section 6 is the heart of the paper, it focusses on the combinatorial modulus on boundaries of hyperbolic Coxeter groups. Section 7 discusses a proof of the Cannon's conjecture in the Coxeter group case. An application to Coxeter groups with Sierpinski carpet boundary is given. Section 8 establishes a sufficient condition for a Coxeter boundary to satisfy the combinatorial Loewner property. Examples are presented in Section 9. Section 10 discusses applications to $\ell_{p}$-equivalence relations.

1.5. Suggestions to the reader. Readers who are concerned only with the Coxeter group case of the Cannon's conjecture may read Section 5, Subsection 2.1, Section 3 until Corollary 3.5, and Sections 6 and 7.

Acknowledgements. We would like to thank Mario Bonk and Frederic Haglund for several helpful discussions on the subject of this article. Special thanks to Peter Haïssinsky for his interest and for several comments on a first version of the paper. We thank the referee for his/her numerous and very valuable suggestions. M. B. was partially supported by ANR grant "Cannon", and B. K. by NSF grant DMS-0701515.

Notation and conventions. Any curve $\gamma:[a, b] \rightarrow X$ is assumed to be continuous. Often we do not distinguish between $\gamma$ and its image $\gamma([a, b])$. Two real valued functions $f, g$ defined on a space $X$ are said to be comparable, and then we write $f \asymp g$, if there exists a constant $C>0$ such that $C^{-1} f \leq g \leq C f$. We write $f \lesssim g$ if there is a constant $C>0$ such that $f \leq C g$. A continuum is a non-empty compact connected topological space, it is non-degenerate if it contains more than one point. For an open ball $B=B(x, r)$ in a metric space and for $\lambda>0$, we denote by $\lambda B$ the ball $B(x, \lambda r)$. The radius of a ball $B$ is denoted by $r(B)$. The open $r$-neighborhood of a subset $E$ is denoted by $N_{r}(E)$. A subset $E$ of a set $F$ is called a proper subset of $F$ if $E \varsubsetneqq F$. A subset $E$ of a geodesic metric space $X$ is convex if for every $x, y \in E$, all the geodesic segments in $X$ between $x$ and $y$ are contained in $E$. Given a graph $G$, we define $G^{0}$ and $G^{1}$ to be the set of vertices and of (non-oriented) open edges respectively. The inclusion of a subgroup $H$ in a group $\Gamma$ is denoted by $H \leqq \Gamma$.

\section{Combinatorial modulus}

This section develops the theory of combinatorial modulus in a general setting. The combinatorial Loewner property and related topics are discussed. For the classical notions of geometric function theory used in this paper, we refer to [Hei01], [V̈̈5], [BK02]. 
Versions of combinatorial modulus have been considered by several authors in connection with Cannon's conjecture on groups with 2-sphere boundary (see e.g. [Can94], [CS98], [CFP99], [BK02], [Hai09]), and in a more general context [Pan89b], [Tys98].

2.1. Definitions and first properties. Let $(Z, d)$ be a compact metric space, let $k \in \mathbb{N}$, and let $\kappa \geq 1$. A finite graph $G_{k}$ is called a $\kappa$-approximation of $Z$ on scale $k$, if it is the incidence graph of a covering of $Z$ such that for every $v \in G_{k}^{0}$ there exists $z_{v} \in Z$ with

$$
B\left(z_{v}, \kappa^{-1} 2^{-k}\right) \subset v \subset B\left(z_{v}, \kappa 2^{-k}\right),
$$

and for $v, w \in G_{k}^{0}$ with $v \neq w$,

$$
B\left(z_{v}, \kappa^{-1} 2^{-k}\right) \cap B\left(z_{w}, \kappa^{-1} 2^{-k}\right)=\emptyset .
$$

Note that we identify every vertex $v$ of $G_{k}$ with the corresponding subset in $Z$. A collection of graphs $\left\{G_{k}\right\}_{k \in \mathbb{N}}$ is called a $\kappa$-approximation of $Z$, if for each $k \in \mathbb{N}$ the graph $G_{k}$ is a $\kappa$-approximation of $Z$ on scale $k$.

Let $\gamma \subset Z$ be a curve (or a subset) and let $\rho: G_{k}^{0} \rightarrow \mathbb{R}_{+}$be any function. The $\rho$-length of $\gamma$ is

$$
L_{\rho}(\gamma)=\sum_{v \cap \gamma \neq \emptyset} \rho(v) .
$$

For $p \geq 1$ the $p$-mass of $\rho$ is

$$
M_{p}(\rho)=\sum_{v \in G_{k}^{0}} \rho(v)^{p} .
$$

Let $\mathcal{F}$ be a non-void family of curves in $Z$, we define its $G_{k}$-combinatorial $p$-modulus by

$$
\operatorname{Mod}_{p}\left(\mathcal{F}, G_{k}\right)=\inf _{\rho} M_{p}(\rho)
$$

where the infimum is over all $\mathcal{F}$-admissible functions i.e. functions $\rho: G_{k}^{0} \rightarrow \mathbb{R}_{+}$ which satisfy $L_{\rho}(\gamma) \geq 1$ for every $\gamma \in \mathscr{F}$. If $\mathscr{F}=\emptyset$ we $\operatorname{set} \operatorname{Mod}_{p}\left(\mathscr{F}, G_{k}\right)=0$. Observe that admissible functions with minimal $p$-mass are smaller than or equal to 1 .

We denote by $\mathcal{F}(A, B)$ the family of curves joining two subsets $A$ and $B$ of $Z$ and by $\operatorname{Mod}_{p}\left(A, B, G_{k}\right)$ its $G_{k}$-combinatorial $p$-modulus. The following properties are routine to verify.

Proposition 2.1. (1) If $\mathscr{F}_{1} \subset \mathscr{F}_{2}$ then $\operatorname{Mod}_{p}\left(\mathcal{F}_{1}, G_{k}\right) \leq \operatorname{Mod}_{p}\left(\mathscr{F}_{2}, G_{k}\right)$.

(2) If $\mathcal{F}_{1}, \ldots, \mathcal{F}_{n}$ be curves families, then

$$
\operatorname{Mod}_{p}\left(\cup \mathcal{F}_{j}, G_{k}\right) \leq \sum \operatorname{Mod}_{p}\left(\mathcal{F}_{j}, G_{k}\right) .
$$

(3) Let $\widetilde{F}_{1}, \mathcal{F}_{2}$ be two curve families. Suppose that each curve in $\mathcal{F}_{1}$ admits a subcurve in $\mathscr{F}_{2}$, then $\operatorname{Mod}_{p}\left(\mathscr{F}_{1}, G_{k}\right) \leq \operatorname{Mod}_{p}\left(\mathscr{F}_{2}, G_{k}\right)$. 
Recall that a metric space $Z$ is called a doubling metric space if there is a constant $n \in \mathbb{N}$ such that every ball $B$ can be covered by at most $n$ balls of radius $\frac{r(B)}{2}$. For a doubling metric space the combinatorial modulus does not depend on the choice of the graph approximation up to a multiplicative constant. More precisely we have:

Proposition 2.2. Assume that $Z$ is a doubling metric space. Then for every $\kappa, \kappa^{\prime} \geq 1$ and every $p \geq 1$ there exists a constant $D \geq 1$ such that for any $k \in \mathbb{N}$ and for any graphs $G_{k}, G_{k}^{\prime}$ which are respectively $\kappa$ and $\kappa^{\prime}$-approximations of $Z$ on scale $k$, one has

$$
D^{-1} \operatorname{Mod}_{p}\left(\cdot, G_{k}\right) \leq \operatorname{Mod}_{p}\left(\cdot, G_{k}^{\prime}\right) \leq D \operatorname{Mod}_{p}\left(\cdot, G_{k}\right)
$$

Proof. The doubling property allows one to bound the maximal number $N$ of pieces of $G_{k}^{0}$ which overlap a given piece of $G_{k}^{\prime 0}$, in terms of $n, \kappa, \kappa^{\prime}$.

Let $\mathcal{F}$ be a family of curves in $Z$. For every $\mathcal{F}$-admissible function $\rho: G_{k}^{0} \rightarrow \mathbb{R}_{+}$ define $\rho^{\prime}: G_{k}^{\prime 0} \rightarrow \mathbb{R}_{+}$by

$$
\rho^{\prime}\left(v^{\prime}\right)=\max \left\{\rho(v) ; v \in G_{k}^{0}, v \cap v^{\prime} \neq \emptyset\right\} .
$$

For $v^{\prime} \in G_{k}^{\prime 0}$ one has

$$
N \rho^{\prime}\left(v^{\prime}\right) \geq \sum_{v \cap v^{\prime} \neq \emptyset} \rho(v) .
$$

Hence $N \rho^{\prime}$ is an $\mathcal{F}$-admissible function for the graph $G_{k}^{\prime}$. In addition, letting $N^{\prime}$ be the maximal number of pieces of $G_{k}^{\prime 0}$ which overlap a given piece of $G_{k}^{0}$, we have

$$
M_{p}\left(\rho^{\prime}\right) \leq \sum_{v^{\prime} \in G_{k}^{\prime 0}} \sum_{v \cap v^{\prime} \neq \emptyset} \rho(v)^{p} \leq N^{\prime} M_{p}(\rho)
$$

so we get

$$
\operatorname{Mod}_{p}\left(\mathcal{F}, G_{k}^{\prime}\right) \leq N^{p} N^{\prime} \operatorname{Mod}_{p}\left(\mathcal{F}, G_{k}\right)
$$

The following lemma is sometimes useful to understand the asymptotic behaviour of a minimal admissible function when $k$ tends to $+\infty$.

Lemma 2.3. Let $\mathcal{F}$ be a curve family in $Z$, let $G_{k}$ be a $\kappa$-approximation of $Z$ on

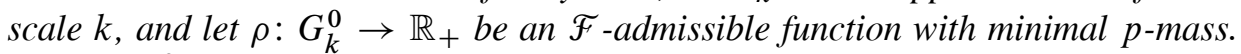
For $v \in G_{k}^{0}$ define

$$
\mathscr{F}_{v}=\{\gamma \in \mathscr{F} ; \gamma \cap v \neq \emptyset\} .
$$

Then one has $\rho(v) \leq \operatorname{Mod}_{p}\left(\mathcal{F}_{v}, G_{k}\right)^{1 / p}$.

Proof. Let $\rho_{v}: G_{k}^{0} \rightarrow \mathbb{R}_{+}$be a minimal $\mathcal{F}_{v}$-admissible function, and let $\tilde{\rho}$ be the function on $G_{k}^{0}$ defined by $\tilde{\rho}(w)=\max \left\{\rho(w), \rho_{v}(w)\right\}$ for $w \neq v$, and by $\tilde{\rho}(v)=$ $\rho_{v}(v)$. Clearly $\tilde{\rho}$ is an $\mathcal{F}$-admissible function, thus:

$$
\operatorname{Mod}_{p}\left(\widetilde{F}, G_{k}\right) \leq M_{p}(\tilde{\rho}) \leq \sum_{w \neq v} \rho(w)^{p}+\sum_{w} \rho_{v}(w)^{p},
$$


which implies that

$$
\operatorname{Mod}_{p}\left(\mathscr{F}, G_{k}\right) \leq \operatorname{Mod}_{p}\left(\widetilde{F}, G_{k}\right)-\rho(v)^{p}+\operatorname{Mod}_{p}\left(\mathscr{F}_{v}, G_{k}\right)
$$

The statement follows.

2.2. The combinatorial Loewner property (CLP). In this subsection we define a combinatorial analog of the Loewner property introduced by J. Heinonen and P. Koskela in [HK98]. This notion appears in [Kle06], Section 7. We will show that the CLP has a number of features in common with the Loewner property. Examples of spaces satisfying the combinatorial Loewner property will be given in Sections 9 and 4.

We assume that $Z$ is a compact arcwise connected doubling metric space, in particular Proposition 2.2 holds. Let $\left\{G_{k}\right\}_{k \in \mathbb{N}}$ be a $\kappa$-approximation of $Z$. Recall that the relative distance between two disjoint non-degenerate continua $A, B \subset Z$ is

$$
\Delta(A, B)=\frac{\operatorname{dist}(A, B)}{\min \{\operatorname{diam} A, \operatorname{diam} B\}} .
$$

Definition 2.4. Suppose $p>1$. Then $Z$ satisfies the combinatorial $p$-Loewner property if there exist two positive increasing functions $\phi, \psi$ on $(0,+\infty)$ with $\lim _{t \rightarrow 0} \psi(t)=0$ such that for all disjoint non-degenerate continua $A, B \subset Z$ and for all $k$ with $2^{-k} \leq \min \{\operatorname{diam} A$, diam $B\}$ one has

$$
\phi\left(\Delta(A, B)^{-1}\right) \leq \operatorname{Mod}_{p}\left(A, B, G_{k}\right) \leq \psi\left(\Delta(A, B)^{-1}\right) .
$$

We say that $Z$ satisfies the combinatorial Loewner property if it satisfies the combinatorial $p$-Loewner property for some $p>1$.

Note that thanks to Proposition 2.2, the definition does not depend on the choice of the $\kappa$-approximation.

The following properties have been established by J. Heinonen and P. Koskela for Loewner spaces (see [HK98], Theorem 3.13 and Remark 3.19). Their proof generalizes verbatim to the spaces which satisfy the combinatorial Loewner property.

Proposition 2.5. Assume that $Z$ satisfies the CLP, then the following holds:

(1) $Z$ is linearly connected, in other words there exists a constant $C \geq 1$ such that any two points $z_{1}, z_{2} \in Z$ can be joined by a path of diameter less or equal to $C d\left(z_{1}, z_{2}\right)$.

(2) Z has no local cut point, in other words no connected open subset is disconnected by removing a point.

We also have: 
Theorem 2.6. (1) If $Z$ is a compact Ahlfors p-regular, p-Loewner metric space, then $Z$ satisfies the combinatorial $p$-Loewner property.

(2) If $Z^{\prime}$ is quasi-Möbius homeomorphic to a compact space $Z$ satisfying the CLP, then $Z^{\prime}$ also satisfies the CLP (with the same exponent).

The proof of (1) involves transferring admissible functions on the metric measure space to admissible functions on an associated discrete approximation, and viceversa. The arguments are straightforward imitations of those appearing in [HK98], [BK02], [Hai09], so we omit them.

The proof of (2) is similar in spirit, except that the admissible functions are transferred between two discrete approximations. It involves some of the techniques that will be used frequently in the sequel.

Proof of (2). We start by some general observations. Let $f: Z \rightarrow Z^{\prime}$ be a quasiMöbius homeomorphism. Since $Z$ and $Z^{\prime}$ are bounded, $f$ is a quasi-symmetric homeomorphism (see [V85]). The doubling property and linear connectedness are preserved by quasi-symmetric homeomorphisms. Since $Z$ is a doubling linearly connected space (see Prop. 2.5), $Z^{\prime}$ is also. Given a $\kappa^{\prime}$-approximation $G_{\ell}^{\prime}$ of $Z^{\prime}$, the preimages $f^{-1}\left(v^{\prime}\right)$ of the pieces $v^{\prime} \in G_{\ell}^{\prime 0}$ form a covering of $Z$ that we denote by $U_{\ell}$. It enjoys the following properties: there exist constants $\lambda \geq 1$ and $N \in \mathbb{N}$, depending only on $\kappa^{\prime}$, on the doubling constants of $Z, Z^{\prime}$, and on the quasi-symmetric parameters of $f$, such that the following holds:

(i) For every $u \in \mathcal{U}_{\ell}$, there is a ball $B_{u} \subset Z$ with $\frac{1}{\lambda} B_{u} \subset u \subset B_{u}$.

(ii) For every $z \in Z$, the number of balls $B_{u}$ containing $z$ is bounded by $N$.

(iii) If $2 B_{u}$ and $2 B_{v}$ intersect, their radii satisfy $r\left(B_{u}\right) \leq \lambda r\left(B_{v}\right)$.

For disjoint continua $E, F \subset Z$ one defines in an obvious way their $p$-modulus relative to the covering $U_{\ell}$, denoted by $\operatorname{Mod}_{p}\left(E, F, U_{\ell}\right)$, so that $\operatorname{Mod}_{p}\left(E, F, U_{\ell}\right)=$ $\operatorname{Mod}_{p}\left(f(E), f(F), G_{\ell}^{\prime}\right)$.

Assuming that $Z$ satisfies the combinatorial $p$-Loewner property, we will compare $\operatorname{Mod}_{p}\left(E, F, U_{\ell}\right)$ and $\operatorname{Mod}_{p}\left(E, F, G_{k}\right)$ for $k \gg \ell$. The statement (2) will follow since, for a quasi-Möbius homeomorphism $f$, the relative distances $\Delta(E, F)$ and $\Delta(f(E), f(F))$ are quantitatively related (see [BK02], Lemma 3.2). We begin with the

Left-hand side CLP inequalities. We are looking for a positive increasing function $\phi^{\prime}$ on $(0,+\infty)$ such that for all disjoint non-degenerate continua $E, F \subset Z$, and all $\ell$ with $2^{-\ell} \leq \min \{\operatorname{diam} f(E), \operatorname{diam} f(F)\}$ :

$$
\phi^{\prime}\left(\Delta(f(E), f(F))^{-1}\right) \leq \operatorname{Mod}_{p}\left(f(E), f(F), G_{\ell}^{\prime}\right) .
$$

We may assume that $2^{-\ell}$ is small in comparison with $\operatorname{dist}(f(E), f(F))$. Indeed, in the contrary, there is a family of curves joining $f(E)$ and $f(F)$, which lies in the union of a controlled amount of pieces of $G_{\ell}^{\prime}$ (recall that $Z^{\prime}$ is a doubling linearly 
connected space). Its $G_{\ell}^{\prime}$-modulus is bounded from below in terms of the number of these pieces. Thus, via the correspondence induced by $f$, we may assume in addition that:

(iv) None of the $2 B_{u}\left(u \in U_{\ell}\right)$ intersects both continua $E, F$.

We will show the existence of a constant $C \geq 0$ such that for every $E, F, \ell$ as above and for all $k \gg \ell$, one has

$$
\operatorname{Mod}_{p}\left(E, F, G_{k}\right) \leq C \operatorname{Mod}_{p}\left(E, F, U_{\ell}\right) .
$$

As explained in the first part of the proof, inequality (2.1) will follow. Let $\varphi: U_{\ell} \rightarrow$ $\mathbb{R}_{+}$be an $\mathcal{F}(E, F)$-admissible function. For $k \gg \ell$ we wish to define an $\mathcal{F}(E, F)$ admissible function of $G_{k}^{0}$ whose $p$-mass is controlled from above by $M_{p}(\varphi)$. To this aim, for every $u \in U_{\ell}$, consider a minimal $\mathcal{F}\left(\overline{B_{u}}, Z \backslash \frac{3}{2} B_{u}\right)$-admissible function $\rho_{u}: G_{k}^{0} \rightarrow \mathbb{R}_{+}$, and let $\rho_{k}: G_{k}^{0} \rightarrow \mathbb{R}_{+}$be defined by

$$
\rho_{k}(v)=\sum_{u \in \mathcal{U}} \varphi(u) \rho_{u}(v) \quad \text { for all } v \in G_{k}^{0} .
$$

For every $\gamma \in \mathcal{F}(E, F)$, one has with (iv) and the definition of $\rho_{u}$

$$
1 \leq \sum_{u \cap \gamma \neq \emptyset} \varphi(u) \leq \sum_{u \cap \gamma \neq \emptyset} \varphi(u)\left(\sum_{v \cap \gamma \cap 2 B_{u} \neq \emptyset} \rho_{u}(v)\right) .
$$

Using property (ii) we obtain that $N \rho_{k}$ is $\mathcal{F}(E, F)$-admissible.

To estimate $p$-masses, observe that $\rho_{u}$ being minimal, it is supported on the set of $v \in G_{k}^{0}$ such that $v \subset 2 B_{u}$. Moreover its $p$-mass is smaller than $\psi(1 / 2)$ since $Z$ satisfies the CLP. In combination with (ii), these properties show that the $p$-mass of $\rho_{k}$ is

$$
\sum_{v \in G_{k}^{0}}\left(\sum_{u \in \mathcal{U}, v \subset 2 B_{u}} \varphi(u) \rho_{u}(v)\right)^{p} \leq N^{p-1} \sum_{v \in G_{k}^{0}} \sum_{u \in \mathcal{U}} \varphi(u)^{p} \rho_{u}(v)^{p},
$$

which is less than $N^{p-1} \psi(1 / 2) M_{p}(\varphi)$. Therefore inequality 2.2 holds with $C=$ $N^{2 p-1} \psi(1 / 2)$. It remains to establish the

Right-hand side CLP inequalities. We are looking for a positive increasing function $\psi^{\prime}$ on $(0,+\infty)$, with $\lim _{t \rightarrow 0} \psi^{\prime}(t)=0$ such that for all disjoint non-degenerate continua $E, F \subset Z$ and all $\ell$ with $2^{-\ell} \leq \min \{\operatorname{diam} f(E)$, diam $f(F)\}$, one has

$$
\operatorname{Mod}_{p}\left(f(E), f(F), G_{\ell}^{\prime}\right) \leq \psi^{\prime}\left(\Delta(f(E), f(F))^{-1}\right) .
$$

As explained in the first part of the proof, it is enough to find a constant $D \geq 0$ such that for all $E, F, \ell$ as above, and for all $k \gg \ell$,

$$
\operatorname{Mod}_{p}\left(E, F, U_{\ell}\right) \leq D \operatorname{Mod}_{p}\left(E, F, G_{k}\right) .
$$

It requires the following general observation, that will also be used frequently in the sequel: 
Lemma 2.7. let $\mathcal{F}$ be a family of curves in a general compact metric space $Z$ and let $M>0$. Then $\operatorname{Mod}_{p}\left(\mathcal{F}, G_{k}\right) \geq M$ if and only if for every function $\rho: G_{k}^{0} \rightarrow \mathbb{R}_{+}$ there exists a curve $\gamma \in \mathscr{F}$ with

$$
L_{\rho}(\gamma) \leq\left(\frac{M_{p}(\rho)}{M}\right)^{1 / p}
$$

Proof. Just notice that the modulus can be written as

$$
\operatorname{Mod}_{p}\left(\mathcal{F}, G_{k}\right)=\inf _{\rho} \frac{M_{p}(\rho)}{L_{\rho}(\mathscr{F})^{p}} \quad \text { with } L_{\rho}(\mathcal{F})=\inf _{\gamma \in \mathcal{F}} L_{\rho}(\gamma),
$$

where $\rho$ is any positive function on $G_{k}^{0}$.

Let $k \gg \ell$ and let $\rho_{k}: G_{k}^{0} \rightarrow \mathbb{R}_{+}$be any function. According to Lemma 2.7, to establish inequality (2.3), it is enough to construct a curve $\gamma \in \mathcal{F}(E, F)$ whose $\rho_{k}$-length is controlled from above by

$$
\left(\frac{M_{p}\left(\rho_{k}\right)}{\operatorname{Mod}_{p}\left(E, F, U_{\ell}\right)}\right)^{1 / p}
$$

Recall that $2^{-\ell} \leq \min \{\operatorname{diam} f(E)$, diam $f(F)\}$. Increasing $\lambda$ if necessary, it yields the following:

(v) Whenever $B_{u}$ intersects $E$, the diameter of $2 B_{u} \cap E$ is larger than $\frac{1}{\lambda} r\left(B_{u}\right)$ (and the same holds for $F$ too).

Let $\Lambda \geq 1$ be a constant (that will be specified later on), and let $\varphi: U_{\ell} \rightarrow \mathbb{R}_{+}$be the function

$$
\varphi(u)=\left(\sum_{v \cap 2 \Lambda B_{u} \neq \emptyset} \rho_{k}(v)^{p}\right)^{1 / p} \text { for all } u \in \mathcal{U}_{\ell} .
$$

The obvious generalization of property (ii) implies that

$$
M_{p}\left(\rho_{k}\right) \asymp M_{p}(\varphi) .
$$

Lemma 2.7 shows that there is a curve $\delta \in \mathcal{F}(E, F)$ such that

$$
L_{\varphi}(\delta) \leq\left(\frac{M_{p}(\varphi)}{\operatorname{Mod}_{p}\left(E, F, U_{\ell}\right)}\right)^{1 / p}
$$

Let $u_{i} \in U_{\ell}$ so that $\delta$ enters successively $u_{1}, \ldots, u_{n}$, and set $B_{i}:=B_{u_{i}}$ for simplicity. We will now use the following lemma whose proof is similar to the one of Lemma 3.17 in [HK98].

Lemma 2.8. Let $Z$ be a compact metric space satisfying the CLP. Then for every $\alpha \in(0,1)$ there exist constants $\Lambda \geq 1$ and $m>0$ such that for every ball $B \subset Z$ and 
every disjoint continua $E_{1}, E_{2} \subset B$ with diam $E_{i} \geq \alpha r(B)$, the $G_{k}$-combinatorial p-modulus of the family

$$
\left\{\eta \in \mathcal{F}\left(E_{1}, E_{2}\right) ; \eta \subset \Lambda B\right\}
$$

is greater than $m$, for every $k$ with $2^{-k} \leq \min \left\{\operatorname{diam} E_{1}\right.$, diam $\left.E_{2}\right\}$.

This lemma in combination with Lemma 2.7 and properties (iii), (v), allows one to construct by induction on $s \in\{1, \ldots, n-1\}$ a curve $\gamma_{s} \subset \bigcup_{i=1}^{s} 2 \Lambda B_{i}$, joining $E$ to $B_{s+1}$, whose $\rho_{k}$-length is bounded linearly from above by $\sum_{i=1}^{s} \varphi\left(u_{i}\right)$. Indeed this follows from letting $\alpha=\frac{1}{2 \lambda}, \Lambda=\Lambda(\alpha)$ and $B=2 B_{s}$ in the statement of the above lemma. A step further gives a curve $\gamma \in \mathcal{F}(E, F)$ whose $\rho_{k}$-length is bounded linearly from above by $L_{\varphi}(\delta)$. Thanks to the estimates (2.4) and (2.5), the curve $\gamma$ enjoys the expected properties. The theorem follows.

2.3. Combinatorial ball Loewner condition. Our next result is a combinatorial version of Proposition 3.1 in [BK05a]. It asserts that a space that satisfies a combinatorial Loewner type condition for pairs of balls satisfies the combinatorial Loewner condition for all pairs of continua. It is a main tool to exhibit examples of spaces satisfying the combinatorial Loewner property.

Proposition 2.9. Let $p \geq 1$. Assume that for every $A>0$ there exist constants $m=m(A)>0$ and $L=L(A)>0$ such that if $r>0$ and $B_{1}, B_{2} \subset Z$ are $r$-balls with $\operatorname{dist}\left(B_{1}, B_{2}\right) \leq A r$, then for every $k \geq 0$ with $2^{-k} \leq r$ the $G_{k}$-combinatorial p-modulus of the family

$$
\left\{\gamma \in \mathcal{F}\left(B_{1}, B_{2}\right) ; \operatorname{diam}(\gamma) \leq L r\right\}
$$

is greater than $m$. Then there exists a positive increasing function $\phi$ of $(0,+\infty)$ such that for every disjoint non-degenerate continua $E_{1}, E_{2} \subset Z$ and for every $k$ with $2^{-k} \leq \min \left\{\operatorname{diam} E_{1}\right.$, diam $\left.E_{2}\right\}$, one has

$$
\phi\left(\Delta\left(E_{1}, E_{2}\right)^{-1}\right) \leq \operatorname{Mod}_{p}\left(E_{1}, E_{2}, G_{k}\right) .
$$

Its proof is a rather straightforward discretization of the proof of Proposition 3.1 in [BK05a]. For the sake of completeness and because similar ideas will be used in Section 8 , we will give the details of the proof. Since it is the most technical part of paper, readers may skip it at the first reading. The proof requires the following lemma which is the analog of Lemma 3.7 in [BK05a].

Lemma 2.10. Let $Z$ be as in Proposition 2.9 and suppose $0<\lambda<1 / 8$. There exist constants $\Lambda=\Lambda(\lambda)$ and $C=C(\lambda)$ with the following property. Let $\rho: G_{k}^{0} \rightarrow \mathbb{R}_{+}$ be any function, let $B=B(z, r)$ be a ball of radius $0<r<\operatorname{diam} Z$, and let $F_{1}, F_{2} \subset Z$ be two continua with $F_{i} \cap \frac{1}{4} B \neq \emptyset$ and $F_{i} \backslash B \neq \emptyset$ for $i=1,2$. Then for $2^{-k+2} \leq \lambda r$ there exist disjoint balls $B_{i}, i=1,2$, and a path $\sigma \subset Z$ satisfying the following properties: 
(i) $B_{1}$ and $B_{2}$ are disjoint, they are centered on $F_{i}$ and of radius $\lambda r$.

(ii) $B_{i} \subset \frac{7}{8} B$ and

$$
\sum_{v \subset B_{i}} \rho(v)^{p} \leq 8 \lambda \sum_{v \subset B} \rho(v)^{p}
$$

(iii) The path $\sigma$ joins $\frac{1}{4} B_{1}$ to $\frac{1}{4} B_{2}$, it is contained in $\Lambda B$ and it has $\rho$-length at most

$$
C \cdot\left(\sum_{v \subset \Lambda B} \rho(v)^{p}\right)^{1 / p}
$$

Proof. We can find a subcontinuum $E_{1} \subset F_{1}$ which is contained in $\bar{B}\left(z, \frac{3 r}{8}\right) \backslash B\left(z, \frac{r}{4}\right)$ and which joins $Z \backslash B\left(z, \frac{3 r}{8}\right)$ to $\bar{B}\left(z, \frac{r}{4}\right)$. Similarly we can find a subcontinuum $E_{2} \subset F_{2}$ which is contained in $\bar{B}\left(z, \frac{3 r}{4}\right) \backslash B\left(z, \frac{5 r}{8}\right)$ and which joins $Z \backslash B\left(z, \frac{3 r}{4}\right)$ to $\bar{B}\left(z, \frac{5 r}{8}\right)$. Then we have $\operatorname{diam} E_{i} \geq r / 8$ for $i=1,2$ and $\operatorname{dist}\left(E_{1}, E_{2}\right) \geq r / 4$.

Since $\operatorname{diam} E_{i} \geq r / 8$, for every $\lambda$ with $0<\lambda<1 / 8$ there exist at least $\frac{1}{8 \lambda}$ pairwise disjoint balls centered on $E_{i}$ and of radius $\lambda r$. So at least one of them called $B_{i}$ - satisfies item (ii) of the statement. The condition (i) is clearly satisfied by the pair of balls $B_{1}, B_{2}$.

Item (iii) follows from the hypothesis on the modulus of curves joining the pair of balls $\frac{1}{4} B_{1}, \frac{1}{4} B_{2}$ and from Lemma 2.7. Note that the radius of $\frac{1}{4} B_{i}$ is equal to $\frac{\lambda r}{4}$ therefore our hypotheses requires that $2^{-k} \leq \frac{\lambda r}{4}$ i.e. $2^{-k+2} \leq \lambda r$.

Proof of Proposition 2.9. Let $\lambda \in \mathbb{R}$ subject to the conditions $0<\lambda<1 / 8$ and $2 \cdot(8 \lambda)^{1 / p}<1$.

Suppose $E_{1}, E_{2} \subset Z$ are disjoint non-degenerate continua and let $\rho: G_{k}^{0} \rightarrow \mathbb{R}_{+}$. According to Lemma 2.7 we are looking for a curve $\gamma$ joining $E_{1}$ to $E_{2}$ whose $\rho$-length is at most

$$
\left(\frac{M_{p}(\rho)}{M}\right)^{1 / p},
$$

where $M>0$ depends only on the relative distance between $E_{1}$ and $E_{2}$.

Pick $p_{i} \in E_{i}$ such that $d\left(p_{1}, p_{2}\right)=\operatorname{dist}\left(E_{1}, E_{2}\right)$. Set

$$
r_{0}:=\frac{1}{2} \min \left\{d\left(p_{1}, p_{2}\right), \operatorname{diam} E_{1}, \operatorname{diam} E_{2}\right\}>0 .
$$

Let $B_{i}=B\left(p_{i}, r_{0}\right)$ for $i=1,2$. Then $B_{1} \cap B_{2}=\emptyset$ and $E_{i} \backslash B_{i} \neq \emptyset$ for $i=1,2$. In addition,

$$
\operatorname{dist}\left(\frac{1}{4} B_{1}, \frac{1}{4} B_{2}\right) \leq\left(\frac{4 d\left(p_{1}, p_{2}\right)}{r_{0}}\right) \frac{r_{0}}{4} \leq t \frac{r_{0}}{4},
$$

where $t:=8 \max \left\{1, \Delta\left(E_{1}, E_{2}\right)\right\}$. By our hypotheses one can find a path $\sigma$ joining $\frac{1}{4} B_{1}$ to $\frac{1}{4} B_{2}$ and whose $\rho$-length is at most $\left(\frac{M_{p}(\rho)}{m}\right)^{1 / p}$ where $m=m(t)$ is the constant appearing in the statement of Proposition 2.9. 
By using Lemma 2.10 inductively, we will construct for successive integers $n$ a family of balls $\mathcal{B}_{n}$, a collection of continua $\Omega_{n}$, and a collection of paths $\Sigma_{n}$ such that the following holds:

(1) $\mathscr{B}_{0}=\left\{B_{1}, B_{2}\right\}, \Omega_{0}=\left\{E_{1}, E_{2}\right\}$ and $\Sigma_{0}=\{\sigma\}$ are defined previously.

(2) For $n \geq 1$ the family $\mathcal{B}_{n}$ consists of $2^{n+1}$ disjoint balls of radius $\lambda^{n} r_{0}$. Each ball $B \in \mathscr{B}_{n}$ is centered on a continuum $\omega \in \Omega_{n}$ with $\omega \backslash B \neq \emptyset$. The collection $\Sigma_{n}$ consists of $2^{n}$ paths, for each element $\sigma \in \Sigma_{n}$ there are exactly two elements $B_{1}, B_{2} \in \mathscr{B}_{n}$ such that $\sigma$ joins $\frac{1}{4} B_{1}$ to $\frac{1}{4} B_{2}$.

The induction proceeds as follows. According to item (2) for every ball $B \in \mathscr{B}_{n}$ there exist $\omega \in \Omega_{n}$ and $\sigma \in \Sigma_{n}$ such that the pair $\left\{F_{1}, F_{2}\right\}:=\{\omega, \sigma\}$ satisfies $F_{i} \cap \frac{1}{4} B \neq \varnothing$ and $F_{i} \backslash B \neq \emptyset$ for $i=1,2$. Thus applying Lemma 2.10 we get two disjoint balls $B_{1}, B_{2}$ and a path $\sigma$ joining $\frac{1}{4} B_{1}$ to $\frac{1}{4} B_{2}$. The balls $B_{i}$ are of radius $\lambda^{n+1} r_{0}$, they are centered respectively on $\omega$ and $\sigma$. The definitions of $\mathscr{B}_{n+1}$ and $\Sigma_{n+1}$ are therefore clear. We define $\Omega_{n+1}$ to be $\Omega_{n} \cup \Sigma_{n}$.

With Lemma 2.10 and by construction the following additional properties are satisfied:

(3) For every ball $B \in \mathscr{B}_{n}$ there exists a ball $B^{\prime} \in \mathscr{B}_{n-1}$ such that $B \subset \frac{7}{8} B^{\prime}$ and

$$
\sum_{v \subset B} \rho(v)^{p} \leq 8 \lambda \sum_{v \subset B^{\prime}} \rho(v)^{p} .
$$

(4) For every path $\sigma \in \Sigma_{n}$ there exists a ball $B^{\prime} \in \mathscr{B}_{n-1}$ such that $\sigma$ lies in $\Lambda B^{\prime}$ and has $\rho$-length at most

$$
C \cdot\left(\sum_{v \subset \Lambda B^{\prime}} \rho(v)^{p}\right)^{1 / p},
$$

where $\Lambda=\Lambda(\lambda)$ and $C=C(\lambda)$.

(5) At each stage $n$ one can index the elements of $\bigcup_{\ell \leq n} \Sigma_{\ell}$ by $\sigma_{1}, \ldots, \sigma_{m}$ and the elements of $\mathcal{B}_{n}$ by $B_{1}, \ldots, B_{m+1}$, in order that $B_{1}$ meets $E_{1}$ and $\sigma_{1}, B_{i}$ meets $\sigma_{i-1}$ and $\sigma_{i}$ for $2 \leq i \leq m$, and $B_{m+1}$ meets $\sigma_{m}$ and $E_{2}$.

We iterate this procedure as long as $n \in \mathbb{N}$ satisfies $2^{-k+2} \leq \lambda^{n} r_{0}$. Let $N$ be the largest integer satisfying this condition.

It remains to connect the paths $\sigma_{i}$ described in item (5) to obtain a curve joining $E_{1}$ to $E_{2}$. For this purpose observe that the hypotheses of Proposition 2.9 assert that for every pair $z_{1}, z_{2}$ of points in $Z$ there exists a path joining $B\left(z_{1}, \frac{1}{4} d\left(z_{1}, z_{2}\right)\right)$ to $B\left(z_{2}, \frac{1}{4} d\left(z_{1}, z_{2}\right)\right)$ whose diameter is comparable to $d\left(z_{1}, z_{2}\right)$. This property implies that every pair of points $z_{1}, z_{2}$ are connected by a path whose diameter is comparable to $d\left(z_{1}, z_{2}\right)$, (see Lemma 3.4 in [BK05a] for more details). Therefore increasing $\Lambda$ if necessary and using item (5) with $n=N$ we exhibit a family $\Theta$ consisting of $2^{N+1}$ curves such that the following holds: 
(6) For every $\theta \in \Theta$ the union of the subsets $v \in G_{k}^{0}$ with $v \cap \theta \neq \varnothing$ is contained in a ball of the form $\Lambda B$ with $B \in \mathcal{B}_{N}$.

(7) The following subset contains a curve $\gamma$ joining $E_{1}$ to $E_{2}$ :

$$
\bigcup_{\theta \in \Theta} \theta \cup \bigcup_{n \leq N} \bigcup_{\sigma \in \Sigma_{n}} \sigma
$$

Observe that for $B \in \mathcal{B}_{N}$ the number of $v \in G_{k}^{0}$ with $v \subset \Lambda B$ is bounded in terms of $\Lambda, \kappa$ and the doubling constant of $Z$. Hence, increasing $C$ if necessary, with property (6) we obtain:

(8) Every $\theta \in \Theta$ has $\rho$-length at most

$$
C \cdot\left(\sum_{v \subset \Lambda B} \rho(v)^{p}\right)^{1 / p}
$$

where $B \in \mathscr{B}_{N}$ is the ball attached to $\theta$ in item (6).

We now compute the $\rho$-length of the curve $\gamma$ defined in (7). At first with properties (2) and (3) one obtains that for $0 \leq s \leq n \leq N$ and for every ball $B \in \mathscr{B}_{n}$ there exists a ball $B^{\prime} \in \mathscr{B}_{n-s}$ such that $\left(\frac{1}{8 \lambda}\right)^{s} \bar{B} \subset \overline{B^{\prime}}$. Let $s=s(\lambda)$ be the smallest integer such that $\left(\frac{1}{8 \lambda}\right)^{s} \geq \Lambda$.

With properties (4) and (3) we get that for $s<n \leq N$ the $\rho$-length of every curve $\sigma \in \Sigma_{n}$ is less than

$$
C \cdot\left((8 \lambda)^{n-s-1} M_{p}(\rho)\right)^{1 / p} .
$$

Similarly with properties (8) and (3) we get that the $\rho$-length of every curve $\theta \in \Theta$ is less than

$$
C \cdot\left((8 \lambda)^{N-s} M_{p}(\rho)\right)^{1 / p} .
$$

For $0<n \leq s$ each $\sigma \in \Sigma_{n}$ has $\rho$-length at most $C\left(M_{p}(\rho)\right)^{1 / p}$. Recall that $\sigma \in \Sigma_{0}$ has $\rho$-length at most $\left(\frac{M_{p}(\rho)}{m}\right)^{1 / p}$. Finally,

$$
L_{\rho}(\gamma) \leq M_{p}(\rho)^{1 / p}\left(\frac{1}{m^{1 / p}}+C \sum_{n=1}^{s} 2^{n}+C \sum_{n=s+1}^{N+1} 2^{n}(8 \lambda)^{(n-s-1) / p}\right) .
$$

Therefore letting $D:=C \cdot(8 \lambda)^{(-s-1) / p}$ and $a:=2 \cdot(8 \lambda)^{1 / p}$ we get

$$
L_{\rho}(\gamma) \leq M_{p}(\rho)^{1 / p}\left(\frac{1}{m^{1 / p}}+D \sum_{n=1}^{N+1} a^{n}\right)
$$

and

$$
M:=\left(\frac{1}{m^{1 / p}}+D \sum_{n=1}^{N+1} a^{n}\right)^{-p}
$$

satisfies the desired properties since $a<1$ by assumption. 
Remarks. 1) We could have chosen to define the CLP using the more general notion of $\kappa$-approximations from [BK02], so as to make the quasi-Möbius invariance automatic from the definition. However, this would simply make it harder to verify in examples, forcing one to prove the equivalence of the two definitions anyway.

2) We emphasize that, unlike the CLP, the classical Loewner property is not, strictly speaking, invariant by quasi-Möbius homeomorphisms. Indeed assume that $Z$ and $Z^{\prime}$ are quasi-Möbius equivalent metric spaces, and that $Z$ is a Loewner space. Then by a theorem of J. Tyson [Tys98], $Z^{\prime}$ is a Loewner space if and only if the Hausdorff dimensions of $Z$ and $Z^{\prime}$ are equal.

For that reason it is in general a difficult problem to decide whether a given metric space is quasi-Möbius equivalent to a Loewner space. The analogous problem for the CLP is in principle easier.

\section{Self-similarity}

This section derives from self-similarity several general principles that will be useful in the sequel. In particular self-similarity permits the reduction of the right-hand side of the CLP inequality to a simpler condition (Proposition 3.4). It also implies a certain asymptotic behaviour for the combinatorial modulus (Proposition 3.6 and Corollary 3.7).

3.1. Approximately self-similar spaces. The following definition appears in Section 3 of [Kle06].

Definition 3.1. A compact metric space $(Z, d)$ is called approximately self-similar if there is a constant $L_{0} \geq 1$ such that if $B(z, r) \subset Z$ is a ball of radius $0<r \leq$ $\operatorname{diam}(Z)$, then there is an open subset $U \subset Z$ which is $L_{0}$-bi-Lipschitz homeomorphic to the rescaled ball $\left(B(z, r), \frac{1}{r} d\right)$.

Observe that approximately self-similar metric spaces are doubling metric spaces. Examples include some classical fractal spaces like the square Sierpinski carpet and the cubical Menger curve (their definitions are recalled in Section 4). A further source of examples comes from expanding Thurston maps, [BM], [HP09]. It follows readily from Corollary 1.5 of $[\mathrm{BM}]$ that with respect to any visual metric as in $[\mathrm{BM}]$, the 2-sphere is approximately self-similar.

We now present examples arising from group actions on boundaries (Proposition 3.3). For this purpose, we first recall some standard notions and results (see e.g. [Gro87], [BH99], [KB02] for more details). Let $X$ be a geodesic proper metric space. Assume that $X$ is hyperbolic (in the sense of Gromov), i.e. there exists a constant $\delta_{X}$, called the triangle fineness constant of $X$, such that for every geodesic triangle $[x, y] \cup[y, z] \cup[z, x] \subset X$ and for every $p \in[x, y]$, one has

$$
\operatorname{dist}(p,[y, z] \cup[z, x]) \leq \delta_{X} .
$$


Denote by $\partial X$ the boundary at infinity of $X$. It carries a visual metric, i.e. a metric $\delta$ for which there are constants $a>1, C \geq 1$ such that for every $z, z^{\prime} \in \partial X$, one has

$$
C^{-1} a^{-\ell} \leq \delta\left(z, z^{\prime}\right) \leq C a^{-\ell}
$$

where $\ell$ denotes the distance from $x_{0}$ (an origin in $X$ ) to a geodesic $\left(z, z^{\prime}\right) \subset X$. Suppose in addition that $\Gamma$ is a (Gromov) hyperbolic group that acts on $X$ by isometries, properly discontinuously and cocompactly. Then $\Gamma$ acts canonically on $(\partial X, \delta)$ by uniformly quasi-Möbius homeomorphisms. Moreover, assuming that $\partial \Gamma$ and $\partial X$ are both equipped with visual metrics, the orbit map $g \in \Gamma \mapsto g x_{0} \in X$ extends to a canonical quasi-Möbius homeomorphism $\partial \Gamma \rightarrow \partial X$. We set:

Definition 3.2. Let $\Gamma$ be a hyperbolic group. A metric $d$ on $\partial \Gamma$ is called a selfsimilar metric, if there exists a hyperbolic geodesic metric space $X$, on which $\Gamma$ acts by isometries, properly discontinuously and cocompactly, such that $d$ is the preimage of a visual metric $\delta$ on $\partial X$ by the canonical homeomorphism $\partial \Gamma \rightarrow \partial X$.

Proposition 3.3. The space $\partial \Gamma$ equipped with a self-similar metric d is approximately self-similar, the partial bi-Lipschitz maps being restrictions of group elements. Moreover $\Gamma$ acts on $(\partial \Gamma, d)$ by (non-uniformly) bi-Lipschitzhomeomorphisms, and $(\partial \Gamma, d)$ is linearly connected as soon as it is connected.

Proof. Let $X$ and $\delta$ be as in Definition 3.2, let $x_{0} \in X$ be an origin and let $\delta_{X}$ be the triangle fineness constant of $X$. Let $a$ and $C$ be the constants associated to $\delta$ and $x_{0}$ by the relations (3.2). We denote the distance in $X$ between $x$ and $y$ by $|x-y|$. We are looking for a constant $L_{0}$ such that for every ball $B(z, r) \subset(\partial X, \delta)$ with $0<r<\operatorname{diam} \partial X$, there is $g \in \Gamma$ whose restriction to $B(z, r)$ is a $L_{0}$-bi-Lipschitz homeomorphism from the rescaled ball $\left(B(z, r), \frac{1}{r} \delta\right)$ onto an open subset of $\partial X$.

Let $B(z, r)$ as above and let $y \in\left[x_{0}, z\right) \subset X$ be such that

$$
\left|x_{0}-y\right|=-\log _{a} 2 r C-\delta_{X} \text {. }
$$

At first we claim that for every $z_{1}, z_{2} \in B(z, r)$ and every $p \in\left(z_{1}, z_{2}\right)$, one has

$$
\operatorname{dist}\left(y,\left[x_{0}, p\right]\right) \leq 3 \delta_{X} .
$$

To see this, notice that $\delta\left(z_{1}, z_{2}\right) \leq 2 r$. Hence from inequalities (3.2) one gets $\operatorname{dist}\left(x_{0},\left(z_{1}, z_{2}\right)\right) \geq-\log _{a} 2 r C$. Let $y_{1} \in\left[x_{0}, z_{1}\right)$ be such that

$$
\left|x_{0}-y_{1}\right|=-\log _{a} 2 r C-\delta_{X} .
$$

One has $\operatorname{dist}\left(y_{1},\left(z_{1}, z_{2}\right)\right) \geq d\left(x_{0},\left(z_{1}, z_{2}\right)\right)-\left|x_{0}-y_{1}\right| \geq \delta_{X}$. For every $p \in\left(z_{1}, z_{2}\right)$ consider the geodesic triangle $\left[x_{0}, z_{1}\right) \cup\left(z_{1}, p\right] \cup\left[p, x_{0}\right]$. With the inequality (3.1) we get $\operatorname{dist}\left(y_{1},\left[x_{0}, p\right]\right) \leq \delta_{X}$. For $z_{2}=z$ and $p=z_{2}$ we obtain in particular that $\left|y-y_{1}\right| \leq 2 \delta_{X}$. The claim follows. 
Let $D$ be the diameter of the quotient space $X / \Gamma$, and let $g \in \Gamma$ be such that $\left|g^{-1} x_{0}-y\right| \leq D$. For any $z_{1}, z_{2} \in B(z, r)$, we deduce from the claim that

$$
\left|\operatorname{dist}\left(g^{-1} x_{0},\left(z_{1}, z_{2}\right)\right)-\operatorname{dist}\left(x_{0},\left(z_{1}, z_{2}\right)\right)-\log _{a} 2 r C\right| \leq D+6 \delta_{X} .
$$

One has $\operatorname{dist}\left(g^{-1} x_{0},\left(z_{1}, z_{2}\right)\right)=\operatorname{dist}\left(x_{0},\left(g z_{1}, g z_{2}\right)\right)$ since $\Gamma$ acts on $X$ by isometries. With inequalities (3.2) it leads to

$$
L_{0}^{-1} \frac{\delta\left(z_{1}, z_{2}\right)}{r} \leq \delta\left(g z_{1}, g z_{2}\right) \leq L_{0} \frac{\delta\left(z_{1}, z_{2}\right)}{r},
$$

where $L_{0}=\frac{C^{3}}{2} a^{D+6 \delta_{X}}$. The first part of the statement follows.

The fact that $\Gamma$ acts on $(\partial X, \delta)$ by (non-uniformly) bi-Lipschitz homeomorphisms can be proved by similar arguments. The connectedness statement is a result from [BK05b].

3.2. Modulus on approximately self-similar spaces. For the rest of the section $Z$ denotes an arcwise connected, approximately self-similar metric space. Let $\left\{G_{k}\right\}_{k \in \mathbb{N}}$ be a $\kappa$-approximation of $Z$. We fix a positive constant $d_{0}$ that is small compared to the diameter of $Z$ and to the constant $L_{0}$ of the Definition 3.1. Denote by $\mathcal{F}_{0}$ the family of curves $\gamma \subset Z$ with $\operatorname{diam}(\gamma) \geq d_{0}$. A first result concerns the right-hand side CLP inequality:

Proposition 3.4. Let $p>1$ and suppose there exists a constant $C \geq 0$ such that for every $k \in \mathbb{N}$ one has $\operatorname{Mod}_{p}\left(\mathcal{F}_{0}, G_{k}\right) \leq C$. Then there exists a positive increasing function $\psi$ of $(0,+\infty)$ with $\lim _{t \rightarrow 0} \psi(t)=0$ such that for every disjoint non-degenerate continua $A, B \subset Z$ and every integer $k$ satisfying $2^{-k} \leq \min \{\operatorname{diam}(A), \operatorname{diam}(B)\}$, one has

$$
\operatorname{Mod}_{p}\left(A, B, G_{k}\right) \leq \psi\left(\Delta(A, B)^{-1}\right) .
$$

Proof. Define

$$
\psi(t)=\sup \operatorname{Mod}_{p}\left(A, B, G_{k}\right) \text { for } t>0,
$$

where the supremum is over all disjoint continua $A, B$ with $\Delta(A, B) \geq 1 / t$ and over all integers $k$ such that $2^{-k} \leq \min \{\operatorname{diam}(A), \operatorname{diam}(B)\}$. From the monotonicity of the modulus (Proposition 2.1.1) and from our hypotheses, the function $t \mapsto \psi(t)$ is non-decreasing with non-negative real values. It is enough to prove that $\psi(t)$ tends to 0 when $t$ tends to 0 .

Let $A$ and $B$ be disjoint non-degenerate continua, assume that $d:=\operatorname{diam}(A)$ is smaller than $\operatorname{diam}(B)$ and let $n$ be the largest integer with $2^{n} \cdot d \leq \operatorname{dist}(A, B)$. Pick $z_{0} \in A$, we get

$$
A \subset B\left(z_{0}, d\right) \text { and } B \subset Z \backslash B\left(z_{0}, 2^{n} \cdot d\right)
$$


For $i \in\{1, \ldots, n-1\}$ define $B_{i}=B\left(z_{0}, 2^{i} \cdot d\right)$. There exists a constant $C_{1}$ depending only on $\kappa$ and on the geometry of $Z$ such that for every $k \in \mathbb{N}$ with $2^{-k} \leq d$ and every $i \in\{1, \ldots, n-1\}$ one has

$$
\operatorname{Mod}_{p}\left(B_{i}, Z \backslash B_{i+1}, G_{k}\right) \leq C_{1} .
$$

Indeed applying the self-similarity property (Definition 3.1) to $B_{i+1}$ we may inflate $\mathcal{F}\left(B_{i}, Z \backslash B_{i+1}\right)$ to a family of curves of essentially unit diameters. Hence the above inequalities follow from the monotonicity of the modulus, from our hypotheses, and from Proposition 2.2.

Choose for every $i \in\{1, \ldots, n-1\}$ a minimal $\mathcal{F}\left(B_{i}, Z \backslash B_{i+1}\right)$-admissible function $\rho_{i}: G_{k}^{0} \rightarrow \mathbb{R}_{+}$, and let $\rho=\frac{1}{n-1} \sum_{i=1}^{n-1} \rho_{i}$. This is an $\mathscr{F}(A, B)$-admissible function since every curve joining $A$ and $B$ joins $B_{i}$ and $Z \backslash B_{i+1}$ too. For $2^{-k} \leq d$ the minimality of the $\rho_{i}$ 's shows that their supports are essentially disjoint, thus

$$
M_{p}(\rho) \leq \frac{C_{2}}{(n-1)^{p}} \sum_{i=1}^{n-1} M_{p}\left(\rho_{i}\right) \leq \frac{C_{1} C_{2}}{(n-1)^{p-1}},
$$

where $C_{2}$ depends only on $\kappa$. Since $p>1$ and $\Delta(A, B) \leq 2^{n+1}$ we get that for $t$ small enough $\psi(t) \leq C_{3}(\log 1 / t)^{-1}$ where $C_{3}$ depends only on $C_{1}, C_{2}, p$.

The following uniformization criterion is a consequence of an uniformization theorem established in [BK02] (see also [CFP99] for related results).

Corollary 3.5. Suppose in addition that $Z$ is homeomorphic to the 2-sphere. Assume that there exists a constant $C \geq 1$ such that for every $k \in \mathbb{N}$ one has

$$
\operatorname{Mod}_{2}\left(\widetilde{F}_{0}, G_{k}\right) \leq C .
$$

Then $Z$ is quasi-Möbius homeomorphic to the Euclidean 2-sphere.

Proof. The statement is a consequence of the previous proposition in combination with [BK02], Theorem 10.4. This theorem supposes that $Z$ is doubling and linearly locally contractible.

The doubling property follows from the fact that $Z$ is approximately self-similar.

Recall that a metric space $Z$ is linearly locally contractible if there exists a constant $\lambda \geq 1$ such that every ball $B \subset Z$ with $0<r(B)<\frac{\text { diam } Z}{\lambda}$ is contractible in $\lambda B$. Approximately self-similar manifolds enjoy this property. Indeed, if $Z$ is a compact manifold, then there is a $R_{0}>0$ and a positive function $\Phi$ of $\left(0, R_{0}\right)$ with $\lim _{t \rightarrow 0} \Phi(t)=0$ such that every ball $B \subset Z$ of radius $r$ with $0<r<R_{0}$, is contractible in $\Phi(r) B$. Let $\lambda \geq 1$ and let $B(z, r) \subset Z$ be a ball with $\lambda r<\operatorname{diam} Z$. Applying self-similarity to the ball $B(z, \lambda r)$, one obtains a open subset $U \subset Z$ and a bilipschitz map $f: B(z, \lambda r) \rightarrow U$ such that for every pair $x, y \in B(z, \lambda r)$ :

$$
L_{0}^{-1} \frac{d(x, y)}{\lambda r} \leq d(f(x), f(y)) \leq L_{0} \frac{d(x, y)}{\lambda r} .
$$


Thus if $\lambda$ satisfies $\frac{L_{0}}{\lambda} \leq L_{0}^{-1}$, one has

$$
f(B(z, r)) \subset B\left(f(z), \frac{L_{0}}{\lambda}\right) \subset B\left(f(z), L_{0}^{-1}\right) \subset f(B(z, \lambda r)) .
$$

Choosing $\lambda$ subject to the conditions $\Phi\left(\frac{L_{0}}{\lambda}\right)<L_{0}^{-1}$ and $\frac{L_{0}}{\lambda} \leq L_{0}^{-1}$, we get that $f(B(z, r))$ is contractible in $f(B(z, \lambda r))$. Therefore so is $B(z, r)$ in $B(z, \lambda r)$.

The assumptions of Theorem 10.4 of [BK02] also require the graphs $G_{k}$ to be (essentially) homeomorphic to 1-skeletons of triangulations of $S^{2}$. Corollary 6.8 of [BK02] ensures existence of such graphs.

We remark finally that when $Z$ is the boundary of a hyperbolic group, the corollary can also be deduced from [CFP99], Theorems 1.5 and 8.2.

We now study the behaviour of $\operatorname{Mod}_{p}\left(\mathcal{F}_{0}, G_{k}\right)$ when $k$ tends to $+\infty$, depending on $p \geq 1$. For this purpose we establish a submultiplicative inequality:

Proposition 3.6. Let $p \geq 1$ and write $M_{k}:=\operatorname{Mod}_{p}\left(\widetilde{F}_{0}, G_{k}\right)$ for simplicity. There exists a constant $C>0$ such that for every pair of integers $k, \ell$ one has

$$
M_{k+\ell} \leq C \cdot M_{k} \cdot M_{\ell}
$$

In addition, when p belongs to a compact subset of $[1,+\infty)$, the constant $C$ may be chosen independent of $p$.

Proof. Let $\rho_{k}: G_{k}^{0} \rightarrow \mathbb{R}_{+}$be a minimal $\mathcal{F}_{0}$-admissible function on scale $k$. By definition each $v \in G_{k}^{0}$ is roughly a ball of $Z$ of radius $2^{-k}$. For every $v \in G_{k}^{0}$, let $B_{v}$ be a ball containing $v$ and whose radius is approximately $2^{-k}$.

We inflate every ball $2 B_{v}$ to essentially unit diameter as in Definition 3.1. Let $g_{v}$ be the corresponding partial bi-Lipschitz map. Define $G_{k+\ell} \cap 2 B_{v}$ to be the incidence graph of the covering of $2 B_{v}$ by the subsets $w \in G_{k+\ell}^{0}$ with $w \cap 2 B_{v} \neq \varnothing$. Using the map $g_{v}$, one can consider the graph $G_{k+\ell} \cap 2 B_{v}$ as a $\left(2 \kappa L_{0}\right)$-approximation of $g_{v}\left(2 B_{v}\right)$ on scale $\ell$. We pull back to $G_{k+\ell} \cap 2 B_{v}$ a normalized minimal $\tilde{F}_{0}$-admissible function on scale $\ell$, in order to get for every $v \in G_{k}^{0}$ a function $\rho_{v}:\left(G_{k+\ell} \cap 2 B_{v}\right)^{0} \rightarrow$ $\mathbb{R}_{+}$with the following properties:

(i) Its $p$-mass is bounded from above by $D \cdot M_{\ell} \cdot \rho_{k}(v)^{p}$, where $D$ is independent of $k$ and $v$.

(ii) Every curve $\gamma \subset 2 B_{v}$ whose diameter is larger than $r\left(B_{v}\right)$ picks up a $\left(\rho_{v}\right)$-length larger than $\rho_{k}(v)$, where $r\left(B_{v}\right)$ is the radius of $B_{v}$.

Define a function $\rho_{k+\ell}$ on $G_{k+\ell}^{0}$ by

$$
\rho_{k+\ell}(w)=\max \rho_{v}(w),
$$

where the maximum is over all $v \in G_{k}^{0}$ with $w \cap 2 B_{v} \neq \emptyset$. Its $p$-mass is linearly bounded from above by

$$
\sum_{v \in G_{k}^{0}} M_{p}\left(\rho_{v}\right)
$$


which in turn is linearly bounded by $M_{p}\left(\rho_{k}\right) \cdot M_{\ell}=M_{k} \cdot M_{\ell}$ (see item (i)).

It remains to prove that $\rho_{k+\ell}$ is an $\mathcal{F}_{0}$-admissible function - up to a multiplicative constant independent of the scale. For $\gamma \in \mathscr{F}_{0}$ we have

$$
1 \leq \sum_{v \cap \gamma \neq \emptyset} \rho_{k}(v) \leq \sum_{v \cap \gamma \neq \emptyset} \sum_{\gamma \cap 2 B_{v} \cap w \neq \emptyset} \rho_{v}(w) .
$$

Indeed the last inequality follows from item (ii) since the relation $v \cap \gamma \neq \emptyset$ implies that there exists a subcurve of $\gamma$ of diameter greater than $r\left(B_{v}\right)$ contained in $2 B_{v}$.

Thus the $\rho_{k+\ell}$-length of $\gamma$ is larger than $1 / N$ where $N$ is maximal number of elements $v \in G_{k}^{0}$ such that $2 B_{v}$ intersects a given piece $w \in G_{k+\ell}^{0}$. Therefore $N \cdot \rho_{k+\ell}$ is $\mathscr{F}_{0}$-admissible.

For every $k \in \mathbb{N}$, observe that $p \mapsto \operatorname{Mod}_{p}\left(\mathcal{F}_{0}, G_{k}\right)$ is a non-increasing continuous function on $[1,+\infty$ ) (monotonicity comes from the fact that minimal admissible functions are smaller than or equal to 1). We define a critical exponent associated to the curve family $\widetilde{F}_{0}$ by

$$
Q_{M}:=\inf \left\{p \in[1,+\infty) ; \lim _{k \rightarrow+\infty} \operatorname{Mod}_{p}\left(\widetilde{F}_{0}, G_{k}\right)=0\right\} .
$$

With Propositions 3.4, 3.6 and Lemma 2.3 one gets

Corollary 3.7. (1) For $p>Q_{M}$ one has $\lim _{k \rightarrow+\infty} \operatorname{Mod}_{p}\left(\mathcal{F}_{0}, G_{k}\right)=0$.

(2) For $1 \leq p \leq Q_{M}$ the sequence $\left\{\operatorname{Mod}_{p}\left(\mathcal{F}_{0}, G_{k}\right)\right\}_{k \geq 0}$ admits a positive lower bound.

(3) If in addition $Z$ is linearly connected, then for $1 \leq p<Q_{M}$ the sequence $\left\{\operatorname{Mod}_{p}\left(\widetilde{F}_{0}, G_{k}\right)\right\}_{k \geq 0}$ is unbounded.

In particular when $Z$ satisfies the combinatorial $p$-Loewner property one has $p=Q_{M}$.

Proof. Part (1) is a consequence of the definition. Part (2) comes from Proposition 3.6 and from the fact that if a positive sequence $\left\{M_{k}\right\}_{k \in \mathbb{N}}$ satisfies $M_{k+\ell} \leq C \cdot M_{k} \cdot M_{\ell}$, then one has

$$
\lim _{k \rightarrow+\infty} M_{k}=0 \Longleftrightarrow \text { there exists } \ell \in \mathbb{N} \text { with } M_{\ell}<C^{-1} .
$$

To establish part (3) suppose by contradiction that the sequence is bounded for some $Q$ with $1<Q<Q_{M}$. Then the conclusion of Proposition 3.4 holds for the exponent $Q$. We will prove that for $p>Q$ the sequence $\left\{\operatorname{Mod}_{p}\left(\mathcal{F}_{0}, G_{k}\right)\right\}_{k \geq 0}$ tends to 0 , contradicting the definition of $Q_{M}$.

Consider a finite covering of $Z$ by balls of radius $\frac{d_{0}}{4}$. Every $\gamma \in \mathcal{F}_{0}$ satisfies $\operatorname{diam}(\gamma) \geq d_{0}$, thus meets at least two disjoint balls of the covering. Therefore, by Proposition 2.1.2, it is enough to show that for every disjoint non-degenerate continua $A$ and $B$ of $Z$ the sequence $\left\{\operatorname{Mod}_{p}\left(A, B, G_{k}\right)\right\}_{k \geq 0}$ tends to 0 . To do so, we 
will establish that the $\mathcal{F}(A, B)$-admissible functions $\rho_{k}: G_{k}^{0} \rightarrow \mathbb{R}_{+}$with minimal $Q$-mass satisfy

$$
\lim _{k \rightarrow+\infty}\left\|\rho_{k}\right\|_{\infty}=0 \quad \text { where }\left\|\rho_{k}\right\|_{\infty}:=\sup _{v \in G_{k}^{0}} \rho_{k}(v) .
$$

For each $v \in G_{k}^{0}$ pick a continuum $E_{v}$ containing $v$ and whose diameter $d_{v}$ is comparable to $\operatorname{diam}(v) \asymp 2^{-k}$. The existence of $E_{v}$ follows from the assumption that $Z$ is linear connected. Any curve in $\mathcal{F}(A, B)$ passing through $v$ possesses a subcurve in each family $\mathscr{F}\left(A, E_{v}\right)$ and $\mathscr{F}\left(B, E_{v}\right)$. Hence with Lemma 2.3 and Proposition 2.1 we get

$$
\rho_{k}(v) \leq \min \left\{\operatorname{Mod}_{Q}\left(A, E_{v}, G_{k}\right), \operatorname{Mod}_{Q}\left(B, E_{v}, G_{k}\right)\right\}^{1 / Q} .
$$

For $2^{-k}$ small enough compared with $\operatorname{dist}(A, B)$ one has

$$
\max \left\{\Delta\left(A, E_{v}\right), \Delta\left(B, E_{v}\right)\right\} \geq \operatorname{dist}(A, B) / 2 d_{v} .
$$

Therefore Proposition 3.4 applied with exponent $Q$ shows that

$$
\rho_{k}(v) \leq \psi\left(2 d_{v} / \operatorname{dist}(A, B)\right)^{1 / Q},
$$

and so $\left\|\rho_{k}\right\|_{\infty}$ tends to 0 when $k$ tends to $+\infty$.

Finally the CLP assertion follows from Proposition 2.5.1.

Remarks. 1) In [HP] the authors have proved that if $Z$ satisfies the combinatorial $Q$-Loewner property then for every disjoint non-degenerate continua $A, B \subset Z$ one has for $1 \leq p<Q$ :

$$
\lim _{k \rightarrow+\infty} \operatorname{Mod}_{p}\left(A, B, G_{k}\right)=+\infty .
$$

2) In $[\mathrm{KeK}] \mathrm{S}$. Keith and the second named author establish that $Q_{M}$ is equal to the Ahlfors regular conformal dimension of $Z$ i.e. the infimum of Hausdorff dimensions of Ahlfors regular metric spaces quasi-Möbius homeomorphic to $Z$. A proof of this equality is also part of the forthcoming $\mathrm{PhD}$ Thesis [Car]. As a consequence $Q_{M}$ is a quasi-Möbius invariant of $Z$.

3) M. Barlow and R. Bass have established submultiplicative and supermultiplicative inequalities for the combinatorial 2-modulus on some self-similar space like the square Sierpinski carpet. Their method relies on the analysis of random walks on graph approximations (see [Bar03], Lemma 3.2).

4) One may formulate a variant of Proposition 3.6 for doubling spaces in general, by modifying the definition of $M_{k}$. For constants $C_{1}, C_{2}$, and each $k$, let $M_{k}$ be the supremum, as $x$ ranges over $Z$, of the $p$-modulus of the family of curves of diameter at least $C_{1} 2^{-k}$ lying in the ball $B\left(x, C_{2} 2^{-k}\right)$. Then for suitably chosen $C_{1}, C_{2}$, one obtains a submultiplicative inequality $M_{k+\ell} \leq C \cdot M_{k} \cdot M_{\ell}$. 


\section{The combinatorial Loewner property for the standard Sierpinski carpet and Menger curve}

The square Sierpinski carpet is the continuum $\mathbb{S} \subset[0,1]^{2} \subset \mathbb{R}^{2}$ constructed as follows: start with the unit square in the plane, subdivide it into nine equal subsquares, remove the middle open square, and then repeat this procedure inductively on the remaining squares. The cubical Menger curve is the continuum

$$
\mathbb{M}=\bigcap_{i=1}^{3} \pi_{i}^{-1}(\mathbb{S}),
$$

where $\pi_{i}: \mathbb{R}^{3} \rightarrow \mathbb{R}^{2}$ is the map which forgets the $i^{\text {th }}$ coordinate. Both $\mathbb{S}$ and $\mathbb{M}$ are endowed with the induced Euclidean metric. This section establishes:

Theorem 4.1. $\mathbb{S}$ and $\mathbb{M}$ satisfy the combinatorial Loewner property.

We notice that similar reasoning applies to other families of self-similar examples, see Subsection 4.3.

Many ideas appearing in this paper have their origin in the analysis of the combinatorial modulus on $\mathbb{S}$ and $\mathbb{M}$. Furthermore, most of the ideas in the proof of Theorem 4.1 will be reused to study the boundaries of Coxeter groups in the next five Sections. We think that this exposition may be useful to the reader to understand the Coxeter group case.

We will treat $\mathbb{S}$ and $\mathbb{M}$ separately. Due to planarity, the Sierpinski carpet case is simpler. One can view the Menger curve case as an intermediate stage between the Sierpinski carpet and Coxeter group boundaries.

4.1. Proof of Theorem 4.1 for the Sierpinski carpet. The proof is split into several steps.

Curves and crossing. We start by analysing the dynamics of the curves in $\mathbb{S}$. Consider the isometric action $D_{4} \curvearrowright[0,1]^{2}$ of the dihedral group $D_{4}$, and let $\Delta \subset[0,1]^{2}$ be the triangular fundamental domain $\left\{(x, y) \in[0,1]^{2} ; y \leq x \leq \frac{1}{2}\right\}$. We identify $\Delta$ with the orbit space $[0,1]^{2} / D_{4}$, and hence we have the orbit map $\pi:[0,1]^{2} \rightarrow \Delta$.

Pick $k \in \mathbb{N}$ and consider the tiling $\mathcal{T}_{k}$ of $\mathbb{S}$ by homothetic copies of itself of side length $3^{-k}$, so that each $T \in \mathcal{T}_{k}$ is contained in a square of the form $\left[\frac{m-1}{3^{k}}, \frac{m}{3^{k}}\right] \times\left[\frac{n-1}{3^{k}}, \frac{n}{3^{k}}\right]$ with $1 \leq m, n \leq 3^{k}$. For every $T \in \mathcal{T}_{k}$, let $\phi_{T}: T \rightarrow \mathbb{S}$ be the homeomorphism which is a composition of a translation and scaling. We have a (continuous) folding map $\pi_{k}: \mathbb{S} \rightarrow \Delta$ whose restriction to each tile $T \in \mathcal{T}_{k}$ is the composition

$$
T \stackrel{\phi_{T}}{\longrightarrow} \mathbb{S} \subset[0,1]^{2} \stackrel{\pi}{\longrightarrow} \Delta .
$$

Lemma 4.2. Suppose $k \in \mathbb{N}$ and $\gamma \subset \mathbb{S}$ is a curve. Then one of the following holds: 
(1) The curve $\pi_{k}(\gamma)$ intersects all three (closed) sides of $\Delta$, and for every (parametrized) curve $\eta \subset \mathbb{S}$ the subset $\pi_{k}^{-1}\left(\pi_{k}(\gamma)\right) \subset \mathbb{S}$ contains a curve which approximates $\eta$ to within error $2 \cdot 3^{-k}$ with respect to the $C^{0}$ distance.

(2) The curve $\gamma$ is disjoint from $\pi_{k}^{-1}(\sigma)$ for one of the (closed) sides $\sigma$ of $\Delta$, and $\operatorname{diam}(\gamma) \leq 2 \cdot 3^{-k}$.

Proof. Suppose the path $\pi_{k}(\gamma)$ intersects all three sides of $\Delta$, and consider elements $g_{1}, g_{2} \in D_{4}$ such $g_{1} \Delta$ and $g_{2} \Delta$ meet along an edge $\sigma$. Then the paths $g_{1} \circ \pi_{k}(\gamma)$ and $g_{2} \circ \pi_{k}(\gamma)$ both touch $\sigma$, and differ by reflection across $\sigma$. Therefore $g_{1} \circ \pi_{k}(\gamma) \cup g_{2} \circ$ $\pi_{k}(\gamma)$ is path connected. It follows that $\delta:=\bigcup_{h \in D_{4}} h \circ \pi_{k}(\gamma)$ is path connected, and that it touches every sides of $[0,1]^{2}$.

Let $\eta \subset \mathbb{S}$ be a curve, and let $T_{1}, \ldots, T_{n}$ be a sequence of tiles in $\mathcal{T}_{k}$ such that every two consecutive tiles share a side, and such that $\eta$ enters successively the $T_{i}$ 's. Then $\bigcup_{i=1}^{n} \phi_{T_{i}}^{-1}(\delta)$ is a subset of $\pi_{k}^{-1}\left(\pi_{k}(\gamma)\right)$. Moreover for every $i=1, \ldots, n-1$ the subsets $\phi_{T_{i}}^{-1}(\delta)$ and $\phi_{T_{i+1}}^{-1}(\delta)$ both meet the side $T_{i} \cap T_{i+1}$ and differ by reflection along $T_{i} \cap T_{i+1}$. Hence $\phi_{T_{i}}^{-1}(\delta) \cup \phi_{T_{i+1}}^{-1}(\delta)$ is path connected. Therefore $\bigcup_{i=1}^{n} \phi_{T_{i}}^{-1}(\delta)$ contains a curve which approximates $\eta$ to within $2 \cdot 3^{-k}$ with respect to the $C^{0}$ distance.

To prove the second part of the alternative we just notice that an Euclidean reflection group generated by a pair of reflections along sides of $\Delta$ fixes one of the vertices of $\Delta$.

Bounds for the combinatorial modulus. We now analyse the combinatorial modulus using the previous lemma. Let $G_{k}$ be the incidence graph of the tiling $\widetilde{T}_{k}$. The collection $\left\{G_{k}\right\}_{k \in \mathbb{N}}$ is a $\kappa$-approximation ${ }^{2}$ of $\mathbb{S}$. As usual we identify every vertex of $G_{k}$ with the corresponding subset in $\mathbb{S}$.

Let $d_{0}$ be a fixed (small) positive constant and let $\mathcal{F}_{0}$ be the family of curves $\gamma \subset \mathbb{S}$ with $\operatorname{diam}(\gamma) \geq d_{0}$. For $\epsilon>0$ and for any (parametrized) curve $\eta$ in $\mathbb{S}$, let $u_{\epsilon}(\eta)$ be the set of curves whose $C^{0}$ distance to $\eta$ is smaller than $\epsilon$.

Lemma 4.3. For every $p \geq 1$ and for every $\epsilon>0$, there exists a constant $C=$ $C(p, \epsilon)$ such that for every non constant curve $\eta \subset \mathbb{S}$ and for every $k \in \mathbb{N}$,

$$
\operatorname{Mod}_{p}\left(\mathcal{F}_{0}, G_{k}\right) \leq C \operatorname{Mod}_{p}\left(U_{\epsilon}(\eta), G_{k}\right)
$$

Furthermore, when $p$ belongs to a compact subset of $[1,+\infty)$, the constant $C$ may be chosen independent of $p$.

Proof. Pick $\ell \in \mathbb{N}$ large enough so that $\epsilon, d_{0} \geq 2 \cdot 3^{-\ell}$. Then, by the previous lemma, for every pair of curves $\eta \subset Z$ and $\gamma \in \widetilde{F}_{0}$ the subset $\pi_{\ell}^{-1}\left(\pi_{\ell}(\gamma)\right) \subset \mathbb{S}$ contains a curve of $U_{\epsilon}(\eta)$.

\footnotetext{
${ }^{2}$ More rigourously there is a subsequence of $\left\{G_{k}\right\}_{k \in \mathbb{N}}$ which is a $\kappa$-approximation of $\mathbb{S}$. Indeed any interval of the form $\left[\frac{1}{2^{k}}, \frac{3}{2^{k}}\right]$ contains a power of $\frac{1}{3}$.
} 
Given $T, T^{\prime} \in \mathcal{T}_{\ell}$ and $h \in D_{4}$, we have an isometry $g_{T, T^{\prime}, h}: T \rightarrow T^{\prime}$ which is the composition of a translation and $\phi_{T}^{-1} \circ h \circ \phi_{T}$. Observe that for every curve $\gamma \subset \mathbb{S}$,

$$
\pi_{\ell}^{-1}\left(\pi_{\ell}(\gamma)\right)=\cup\left\{g_{T, T^{\prime}, h}(\gamma \cap T) ; T, T^{\prime} \in \mathcal{T}_{\ell}, h \in D_{4}\right\} .
$$

Let $k \geq \ell$. For every $U_{\epsilon}(\eta)$-admissible function $\rho: G_{k}^{0} \rightarrow \mathbb{R}_{+}$define a function $\rho^{\prime}: G_{k}^{0} \rightarrow \mathbb{R}_{+}$as follows. For $v \in G_{k}^{0}$, let $T \in \mathcal{T}_{\ell}$ be so that $v \subset T$, and set

$$
\rho^{\prime}(v)=\sum_{T^{\prime}, h} \rho\left(g_{T, T^{\prime}, h}(v)\right)
$$

where $T^{\prime} \in \mathcal{T}_{\ell}$ and $h \in D_{4}$. We claim that $\rho^{\prime}$ is $\mathcal{F}_{0}$-admissible. Indeed let $\gamma \in \mathcal{F}_{0}$, and let $\theta \in U_{\epsilon}(\eta)$ be so that $\theta \subset \pi_{\ell}^{-1}\left(\pi_{\ell}(\gamma)\right)$. One has with (4.1)

$$
L_{\rho^{\prime}}(\gamma)=\sum_{T, T^{\prime}, h} \sum_{\substack{v \subset T \\ v \cap \gamma \neq \emptyset}} \rho\left(g_{T, T^{\prime}, h}(v)\right)=L_{\rho}\left(\pi_{\ell}^{-1}\left(\pi_{\ell}(\gamma)\right) \geq L_{\rho}(\theta) \geq 1 .\right.
$$

The claim follows. Moreover one has

$$
M_{p}\left(\rho^{\prime}\right)=\left|\mathcal{T}_{\ell}\right| \cdot\left|D_{4}\right| \cdot M_{p}(\rho) .
$$

Thus

$$
\operatorname{Mod}_{p}\left(\mathcal{F}_{0}, G_{k}\right) \leq\left|\mathcal{T}_{\ell}\right| \cdot\left|D_{4}\right| \cdot \operatorname{Mod}_{p}\left(U_{\epsilon}(\eta), G_{k}\right) .
$$

Therefore the statement holds for $k \geq \ell$. Since $\ell$ depends only on $\epsilon, d_{0}$, the lemma follows.

Multiplicativity. We now examine the asymptotic behaviour of the modulus $\operatorname{Mod}_{p}\left(\widetilde{F}_{0}, G_{k}\right)$ using the planarity of $\mathbb{S}$. We have

Lemma 4.4. Let $p \geq 1$ and write $M_{k}:=\operatorname{Mod}_{p}\left(\mathscr{F}_{0}, G_{k}\right)$ for simplicity. There exists a constants $C \geq 1$ such that for every pair of integers $k, \ell$ one has

$$
C^{-1} \cdot M_{k} \cdot M_{\ell} \leq M_{k+\ell} \leq C \cdot M_{k} \cdot M_{\ell}
$$

Proof. The right-hand side inequality follows from Proposition 3.6.

To prove the reverse inequality, let $\mathcal{F}_{\mathbb{S}}$ be the family of curves in $\mathbb{S}$ joining the sides $\{0\} \times[0,1]$ and $\{1\} \times[0,1]$. Define $m_{k}:=\operatorname{Mod}_{p}\left(\mathcal{F}_{\mathbb{S}}, G_{k}\right)$. More generally, given a tile $T \in \bigcup_{k \in \mathbb{N}} \mathcal{T}_{k}$, let $\widetilde{F}_{T}$ be the family of curves in $T$ which join the left and right sides of $T$. For $k, \ell \in \mathbb{N}$ and $T \in \mathcal{T}_{k}$ one has: $\operatorname{Mod}_{p}\left(\mathcal{F}_{T}, G_{k+\ell}\right)=m_{\ell}$. We claim that $m_{k} \asymp M_{k}$. Indeed we have $\mathscr{F}_{\mathbb{S}} \subset \mathscr{F}_{0}$, thus $m_{k} \leq M_{k}$. Pick $T:=\left(\left[\frac{1}{3}, \frac{2}{3}\right] \times\left[0, \frac{1}{3}\right]\right) \cap \mathbb{S} \in \mathcal{T}_{1}$ and $\eta:=[0,1] \times\{0\} \subset \mathbb{S}$. Every curve in $\mathcal{U}_{\frac{1}{3}}(\eta)$ admits a subcurve in $\mathcal{F}_{T}$. Therefore, in combination with Lemma 4.3, we obtain

$$
m_{k}=\operatorname{Mod}_{p}\left(\mathcal{F}_{T}, G_{k+1}\right) \geq \operatorname{Mod}_{p}\left(\mathcal{U}_{\frac{1}{3}}(\eta), G_{k+1}\right) \gtrsim M_{k+1} \asymp M_{k} .
$$


The claim follows.

We shall prove that $m_{k+\ell} \gtrsim m_{k} m_{\ell}$. To do so let $\rho_{k+\ell}: G_{k+l}^{0} \rightarrow \mathbb{R}_{+}$be a function. We wish to construct a curve $\gamma \in \mathcal{F}_{\mathbb{S}}$ with controlled $\rho_{k+\ell}$-length. Define $\rho_{k+\ell}^{\prime}: G_{k+\ell}^{0} \rightarrow \mathbb{R}_{+}$as follows. For each $v \in G_{k+\ell}^{0}$, let $T \in \mathcal{T}_{k}$ be so that $v \subset T$, and set $\rho_{k+\ell}^{\prime}(v)$ to be the sum of the $\rho_{k+\ell} \circ \phi(v)$ where $\phi: T \rightarrow T^{\prime}$ ranges over isometries of $T$ with tiles $T^{\prime}$ intersecting $T$.

Pick $T \in \mathcal{T}_{k}$. By Lemma 2.7 there is a curve $\gamma_{T} \in \mathcal{F}_{T}$ such that

$$
L_{\chi_{T} \rho_{k+\ell}^{\prime}}\left(\gamma_{T}\right) \leq\left(\frac{M_{p}\left(\chi_{T} \rho_{k+\ell}^{\prime}\right)}{m_{\ell}}\right)^{\frac{1}{p}},
$$

where $\chi_{T}: G_{k+l}^{0} \rightarrow \mathbb{R}_{+}$is the characteristic function of the set of $v \in G_{k+l}^{0}$ which are contained in $T$. Let $\gamma_{T}^{\prime}$ be the union of the images of $\gamma_{T}$ under all isometries $T \rightarrow T^{\prime}$, where $T^{\prime} \in \mathcal{T}_{k}$ intersects $T$. Then $\gamma_{T}^{\prime}$ is path connected, and

$$
L_{\chi_{T} \rho_{k+\ell}}\left(\gamma_{T}^{\prime}\right)=L_{\chi_{T} \rho_{k+\ell}^{\prime}}\left(\gamma_{T}\right)
$$

Note that if $T_{1}, T_{2} \in \mathcal{T}_{k}$ intersect, then $\gamma_{T_{1}}^{\prime}$ intersects $\gamma_{T_{2}}^{\prime}$, since $\gamma_{T_{2}}^{\prime}$ contains a curve in $T_{1}$ joining the top and bottom, which must intersect $\gamma_{T_{1}}$ by planarity.

Define $\rho_{k}: G_{k}^{0} \rightarrow \mathbb{R}_{+}$by letting $\left(\rho_{k}(T)\right)^{p}$ be the $p$-mass of $\chi_{T} \rho_{k+\ell}^{\prime}$. By Lemma 2.7, there is a vertex path $T_{1}, \ldots, T_{n}$ in $G_{k}$ such that $T_{1}$ intersects $\{0\} \times[0,1]$, $T_{n}$ intersects $\{1\} \times[0,1]$, and whose $\rho_{k}$-length satisfies

$$
\sum_{i} \rho_{k}\left(T_{i}\right) \leq\left(\frac{M_{p}\left(\rho_{k}\right)}{m_{k}}\right)^{\frac{1}{p}}=\left(\frac{M_{p}\left(\rho_{k+\ell}^{\prime}\right)}{m_{k}}\right)^{\frac{1}{p}}
$$

Then the union $\bigcup_{i} \gamma_{T_{i}}^{\prime}$ is a path connected subset of $\mathbb{S}$ joining the left and right sides. Therefore it contains a curve $\gamma \in \mathcal{F}_{\mathbb{S}}$ whose $\rho_{k+\ell}$-length is at most (thanks to (4.3), (4.2) and (4.4)):

$$
\begin{aligned}
\sum_{i} L_{\chi_{T_{i}} \rho_{k+\ell}}\left(\gamma_{T_{i}}^{\prime}\right) & =\sum_{i} L_{\chi_{T_{i}} \rho_{k+\ell}^{\prime}}\left(\gamma_{T_{i}}\right) \\
& \leq \sum_{i}\left(\frac{M_{p}\left(\chi_{T_{i}} \rho_{k+\ell}^{\prime}\right)}{m_{\ell}}\right)^{\frac{1}{p}} \\
& =\sum_{i} \frac{\rho_{k}\left(T_{i}\right)}{m_{\ell}^{1 / p}} \\
& \leq\left(\frac{M_{p}\left(\rho_{k+\ell}^{\prime}\right)}{m_{k} m_{\ell}}\right)^{\frac{1}{p}} \asymp\left(\frac{M_{p}\left(\rho_{k+\ell}\right)}{m_{k} m_{\ell}}\right)^{\frac{1}{p}} .
\end{aligned}
$$

With Lemma 2.7, inequality $m_{k+\ell} \gtrsim m_{k} m_{\ell}$ follows.

Proof of Theorem 4.1 for the Sierpinski carpet, concluded. Let $Q_{M}$ be the critical exponent defined in (3.3). We establish the CLP for $p=Q_{M}$. Lemma 4.4 and the 
proof of Corollary 3.7.2 imply that $M_{k} \asymp 1$ for $p=Q_{M}$. Moreover one has $Q_{M}>1$. Indeed for every $N \in \mathbb{N}$, there is a disjoint collection of paths $\gamma_{1}, \ldots, \gamma_{N} \in \mathscr{F}_{0}$. It follows that with $p=1$ and $k$ large enough, $\operatorname{Mod}_{1}\left(\mathcal{F}_{0}, G_{k}\right) \geq N$. Since $\operatorname{Mod}_{p}\left(\mathscr{F}_{0}, G_{k}\right)$ is bounded for $p=Q_{M}$, we obtain $Q_{M}>1$. Therefore Proposition 3.4 shows that the right-hand side CLP inequality holds. On the other hand, the lower bound $M_{k} \gtrsim 1$, Lemma 4.3 and self-similarity imply that the assumptions of Proposition 2.9 are satisfied. Thus the left-hand side CLP inequality holds.

\subsection{A criterion for a self-similar space to satisfy the CLP, and the proof of}

Theorem 4.1 for the Menger curve. Observe that $\mathbb{M}$ satisfies the obvious analog of Lemma 4.2 with $D_{4}$ replaced by the symmetry group of the cube. Therefore Lemma 4.3 is also valid for $\mathbb{M}$. Moreover, as in the last paragraph, one has (for $p=1)$

$$
\lim _{k \rightarrow+\infty} \operatorname{Mod}_{1}\left(\mathcal{F}_{0}, G_{k}\right)=+\infty .
$$

The following general result implies that $\mathbb{M}$ admits the CLP. Notation is the same as in Lemma 4.3.

Proposition 4.5. Let $Z$ be a linearly connected, approximately self-similar metric space. For $p=1$ assume that $\left\{\operatorname{Mod}_{p}\left(\widetilde{F}_{0}, G_{k}\right)\right\}_{k \in \mathbb{N}}$ is unbounded. Suppose that for every $p \geq 1$, for every non constant curve $\eta \subset Z$ and for every $\epsilon>0$, there exists a constant $C=C(p, \eta, \epsilon)$ such that for every $k \in \mathbb{N}$,

$$
\operatorname{Mod}_{p}\left(\mathcal{F}_{0}, G_{k}\right) \leq C \operatorname{Mod}_{p}\left(\mathcal{U}_{\epsilon}(\eta), G_{k}\right) .
$$

Suppose furthermore that when $p$ belongs to a compact subset of $[1,+\infty)$ the constant $C$ may be chosen independent of $p$. Then $Z$ satisfies the CLP.

The proof uses some arguments that will be discussed in greater generality in Section 8. We refer to this section for more details.

In the following two lemmata the hypotheses of Proposition 4.5 are assumed to hold. The first lemma is a replacement of the supermultiplicativity that was established in Lemma 4.4 using planarity.

Lemma 4.6. Write $M_{k}:=\operatorname{Mod}_{p}\left(\mathcal{F}_{0}, G_{k}\right)$ and $L_{k}:=M_{k}^{-1 / p}$ for simplicity. There exist constants $C \geq 1$ and $b \in(0,1)$ such that for every $k, \ell \in \mathbb{N}$ one has

$$
L_{k+\ell} \leq C \cdot L_{k} \cdot \sum_{n=0}^{\ell} L_{\ell-n} b^{n} .
$$

Moreover when $p$ belongs to a compact subset of $[1,+\infty)$ the constants $C$ and $b$ may be chosen independent of $p$. 
Proof. The assumptions on $Z$ allow one to check that the proofs of Lemmata 8.1, 8.2 and of Proposition 8.3 apply verbatim to $Z$, with $M_{k}$ instead of $m_{k}$. Therefore the inequality holds.

Lemma 4.7. For $p=Q_{M}$ the sequence $\left\{M_{k}\right\}_{k \in \mathbb{N}}$ admits a positive lower bound, and it doesn't tend to $+\infty$.

Proof. The first part of the statement follows from Corollary 3.7.2. The previous lemma shows that the set of $p$ such that $\left\{M_{k}\right\}_{k \in \mathbb{N}}$ tends to $+\infty$ is an open subset of $[1,+\infty)$ - see the proof of Corollary 8.4 for more details. Therefore the second part of the statement holds.

Proof of Proposition 4.5. It is enough to prove that for $p=Q_{M}$ one has $M_{k} \asymp 1$. The $p$-combinatorial Loewner property will then come from Lemma 8.1 and from Propositions 2.9 and 3.4. Note that Proposition 3.4 requires that $p>1$. At this point we use the hypothesis on $\left\{\operatorname{Mod}_{1}\left(\widetilde{F}_{0}, G_{k}\right)\right\}_{k \in \mathbb{N}}$ to ensure that $Q_{M}>1$.

Thanks to Lemma 4.7 the sequence $\left\{M_{k}\right\}_{k \in \mathbb{N}}$ admits a positive lower bound. To obtain the upper bound, one argues exactly as in the second part of the proof of Theorem 8.6, using the fact that $\left\{M_{k}\right\}_{k \in \mathbb{N}}$ does not tend to $+\infty$.

4.3. Other examples. Similar reasoning establishes the CLP for the following examples:

1) Higher dimensional Menger spaces. For $n \geq 2$, the Sierpinski carpet construction may be generalized by iterating the subdivision of the unit cube $[0,1]^{n}$ into $3^{n}$ subcubes of side length $\frac{1}{3}$, and removing the central open subcube. Denote by $\mathbb{S}_{n}$ the resulting space. Then for $\ell \geq n$, we get an analog of the Menger curve by letting

$$
\mathbb{M}_{\ell, n}=\cap\left\{\pi_{I}^{-1}\left(\mathbb{S}_{n}\right) ; I \subset\{1, \ldots, \ell\},|I|=n\right\},
$$

where $\pi_{I}:[0,1]^{\ell} \rightarrow[0,1]^{n}$ is the projection map which retains the coordinates indexed by elements of $I$. These constructions can be further generalized by subdividing into $k^{n}$ subcubes instead of $3^{n}$, where $k$ is an odd integer, or by removing a symmetric pattern of subcubes at each stage, instead of just the central cube, etc.

2) Higher dimensional snowspheres, $c f$. [Mey10]. Fix $n \geq 2$. Let $Q_{k}$ denote the $n$-dimensional polyhedron obtained from the boundary of the cube $\left[0,3^{-k}\right]^{n+1}$ by removing the interior of one $n$-dimensional face. Construct a sequence $\left\{P_{k}\right\}_{k \in \mathbb{Z}_{+}}$, where $P_{k}$ is a metric polyhedron consisting of Euclidean $n$-cubes of side length $3^{-k}$, as follows. Let $P_{0}$ be the boundary of the unit cube $[0,1]^{n}$, and inductively construct $P_{k}$ from $P_{k-1}$ by subdividing each $n$-cube face of $P_{k-1}$ into $3^{n}$ subcubes, removing the central open subcube, and gluing on a copy of $Q_{k}$ along the boundary. If we endow $P_{k}$ with the path metric, then the sequence $\left\{P_{k}\right\}$ Gromov-Hausdorff converges to a self-similar space $Z$. As with Menger spaces, there are further generalizations of these constructions. 
3) Pontryagin manifolds. We modify slightly the above construction to obtain some Pontryagin manifolds. Fix $n \geq 2$, and let $T^{n}$ be the standard $n$-torus obtained by identification of the opposite faces of the unit cube $[0,1]^{n}$. Let $Q_{k}$ denote the polyhedron obtained as follows. Tesselate $T^{n}$ by $3^{n}$ equal subcubes, remove the interior of one of them, and normalize the metric so that the side length of every subcube is $3^{-k}$. Define $P_{0}=T^{n}$ and inductively construct $P_{k}$ from $P_{k-1}$ by subdividing each $n$-cube face of $P_{k-1}$ into $3^{n}$ subcubes, removing the central open subcube, and gluing on a copy of $Q_{k}$ along the boundary. Then, as above, $\left\{P_{k}\right\}$ Gromov-Hausdorff converges to a self-similar space $Z$.

\section{Hyperbolic Coxeter groups}

This section exhibits some specific dynamical properties of the action of a (Gromov) hyperbolic Coxeter group on its boundary. Results of this section will serve to study the combinatorial modulus in Section 6 and the $\ell_{p}$-equivalence relations in Section 10.

5.1. Definitions and first properties. We start by recalling some standard definitions, see [Dav08] for more details. Let $(\Gamma, S)$ be a Coxeter group (see section 1.2 for the definition). A special subgroup of $\Gamma$ is a subgroup generated by a non-empty subset $I$ of $S$; we shall denote it by $\Gamma_{I}$. A parabolic subgroup of $\Gamma$ is a conjugate of a special subgroup. Let $G$ be the Cayley graph of $(\Gamma, S)$. We define $G^{0}$ and $G^{1}$ to be the set of vertices and of (non-oriented) open edges respectively. Each edge carries a type which is an element of $S$. The distance between two vertices $x, y$ of $G$ is denoted by $|x-y|$, and the distance from the identity to $x$ by $|x|$. An edge with endpoints $x, y$ is noted $(x, y)$. For $s \in S$ the wall associated to $s$ is the subset $M_{s} \subset G^{1}$ of $s$-invariant (open) edges. The graph $G \backslash M_{s}$ consists of two disjoint convex closed subsets of $G$, denoted by $H_{-}\left(M_{S}\right)$ and $H_{+}\left(M_{S}\right)$, and called the half-spaces bounded by $M_{s}$. They satisfy the relations

$$
H_{-}\left(M_{s}\right)^{0}=\{g \in \Gamma ;|s g|=|g|+1\}, \quad H_{+}\left(M_{s}\right)^{0}=\{g \in \Gamma ;|s g|=|g|-1\},
$$

and $s$ permutes $H_{-}\left(M_{s}\right)$ and $H_{+}\left(M_{s}\right)$. A wall of $G$ is a subset of the form $g\left(M_{s}\right)$, with $g \in \Gamma$ and $s \in S$. The involution $g s g^{-1}$ is called the reflection along the wall $g\left(M_{s}\right)$. The set of walls forms a partition of $G^{1}$. Each wall $M$ divides $G$ into two disjoint convex closed subsets, called the half-spaces bounded by $M$ and denoted by $H_{-}(M)$ and $H_{+}(M)$, with the convention that $e \in H_{-}(M)$, where $e$ denotes the identity.

Assume now that $\Gamma$ is a hyperbolic Coxeter group (see e.g. [Gro87], [BH99], [KB02] for hyperbolic groups and related topics). We denote by $\partial \Gamma$ its boundary at infinity equipped with a visual metric $d$ (see (3.2)). For a subset $E$ of $G$ we denote by $\partial E$ its limit set in $\partial \Gamma$. A non-empty limit set of a parabolic subgroup of $\Gamma$ will be called a parabolic limit set. If in addition it is a topological circle we shall call it a circular limit set. We notice that: 
Proposition 5.1. For every $\epsilon>0$ there is only a finite number of parabolic limit sets of diameters greater than $\epsilon$.

Proof. Let $P=g \Gamma_{I} g^{-1}$ be a parabolic subgroup with non empty limit set. One has $\partial P=\partial\left(g \Gamma_{I}\right)$. Since $\Gamma$ is a Coxeter group, the inclusion map of the Cayley graph of $\left(\Gamma_{I}, I\right)$ into $G$ is a totally geodesic isometric embedding. By using the visual property (3.2) and the fact that $\Gamma$ acts by isometries on $G$, we obtain that the diameter of $\partial\left(g \Gamma_{I}\right)$ is comparable to $a^{-\operatorname{dist}\left(e, g \Gamma_{I}\right)}$. Only finitely many cosets $g \Gamma_{I}$ intersect a given ball of $G$. The statement follows.

The limit set of a wall $M$ is either of empty interior or equal to $\partial \Gamma$. Indeed this property is well-known for limit sets of subgroups, and the stabilizer of $M$ in $\Gamma$ acts cocompactly on $M$. Using the convexity of the half-spaces one easily sees that

$$
\partial H_{-}(M) \cup \partial H_{+}(M)=\partial \Gamma, \quad \partial H_{-}(M) \cap \partial H_{+}(M)=\partial M .
$$

In consequence, in $\partial \Gamma$, the fixed point set of a reflection is the limit set of its wall.

The following property asserts in particular that the limit sets of half-spaces form a basis of neighborhoods in $\partial \Gamma$.

Proposition 5.2. There exists a constant $\lambda \geq 1$ such that for every $z \in \partial \Gamma$ and every $0<r \leq \operatorname{diam}(\partial \Gamma)$ there exists a half-space $H$ of $G$ with

$$
B\left(z, \lambda^{-1} r\right) \subset \partial H \subset B(z, \lambda r) .
$$

The proof relies on the following lemma. In its statement $\delta_{G}$ denotes the triangle fineness constant of $G$ (see (3.1)).

Lemma 5.3. There exists a constant $L>0$ with the following property. Let $x, x_{1}, y_{1}$, $y$ be four arbitrary points of $G$ lying in this order on a geodesic line. If $\left|x_{1}-y_{1}\right| \geq L$ then there exists a wall $M$ passing between $x_{1}$ and $y_{1}$ such that

$$
\operatorname{dist}(x, M) \geq\left|x-x_{1}\right|-3 \delta_{G} \quad \text { and } \quad \operatorname{dist}(y, M) \geq\left|y-y_{1}\right|-3 \delta_{G} .
$$

Proof. Observe that different edges of a geodesic segment give rise to different walls. Thus there are as many walls passing between $x_{1}$ and $y_{1}$ as edges in $\left[x_{1}, y_{1}\right]$. Let $(a, b)$ be such an edge and let $M$ be the corresponding wall. Let $p \in G^{0}$ be a vertex adjacent to $M$ realizing $\operatorname{dist}(x, M)$. Observe that the geodesic segment $[a, p]$ lies in a $\delta_{G}$-neighborhood of $M$. Indeed, $a$ and $p$ are adjacent to $M$ thus the Hausdorff distance between $[a, p]$ and its image by the reflection along $M$ is smaller than $2 \delta_{G}$, moreover $[a, p]$ and its image are separated by $M$. Therefore we have

$$
\operatorname{dist}(x,[a, p]) \geq|x-p|-\delta_{G} .
$$


Assume that $|x-p|<\left|x-x_{1}\right|-3 \delta_{G}$. Consider the triangle $[x, a] \cup[a, p] \cup[p, x]$ and the point $q \in[p, x]$ with $|q-p|=2 \delta_{G}$. Inequality (5.1) implies that the nearest point projection of $q$ on $[x, a] \cup[a, p]$ lies in $[x, a]$. Thus one gets that

$$
\operatorname{dist}\left(p,\left[x, x_{1}\right]\right) \leq 3 \delta_{G} .
$$

Pick $r \in\left[x, x_{1}\right]$ with $|r-p| \leq 3 \delta_{G}$. Consider the triangle $[r, a] \cup[a, p] \cup[p, r]$, and the nearest point projection of $x_{1}$ on $[a, p] \cup[p, r]$. We obtain that

$$
\operatorname{dist}\left(x_{1},[a, p]\right) \leq 4 \delta_{G} .
$$

Since $[a, p]$ lies in a $\delta_{G}$-neighborhood of $M$, the distance between $M$ and $x_{1}$ is smaller than $5 \delta_{G}$. A similar argument applies to $y$ and $y_{1}$. Let $N$ be the number of walls $M \subset G$ such that $\operatorname{dist}(e, M) \leq 5 \delta_{G}$. The property follows letting $L=2 N+1$.

Proof of Proposition 5.2. Since $d$ is a visual metric, there exist constants $a>1$, $C \geq 1$ such that for every $z, z^{\prime} \in \partial \Gamma$ one has

$$
C^{-1} a^{-\ell} \leq d\left(z, z^{\prime}\right) \leq C a^{-\ell},
$$

where $\ell$ denotes the distance from $e$ (the identity of $\Gamma$ ) to a geodesic $\left(z, z^{\prime}\right) \subset G$. Let $L$ be the constant of the previous lemma. Pick $x_{1}, y_{1}, y$ in this order on the geodesic ray $[e, z) \subset G$, with

$$
\left|x_{1}\right|=-\log _{a} r, \quad\left|y_{1}\right|=-\log _{a} r+L, \quad|y|=-\log _{a} r+L+6 \delta_{G} .
$$

Let $M$ be a wall between $x_{1}$ and $y_{1}$ as in the lemma (applied to $e, x_{1}, y_{1}, y$ ). Set $H:=H_{+}(M)$ for simplicity. By convexity, for every $z^{\prime} \in \partial H$ the geodesic $\left(z, z^{\prime}\right)$ lies in $H$. Therefore dist $\left(e,\left(z, z^{\prime}\right)\right)$ is larger than $-\log _{a} r-3 \delta_{G}$. From (5.2) one obtains that $\partial H \subset B\left(z, C a^{3 \delta_{G}} r\right)$.

To establish the other inclusion, let $z^{\prime} \in \partial \Gamma$ with $\operatorname{dist}\left(e,\left(z, z^{\prime}\right)\right) \geq|y|+3 \delta_{G}$. By the same kind of metric argument as in the first part of the proof of Proposition 3.3, one sees that $\left[e, z^{\prime}\right)$ intersects $B\left(y, 3 \delta_{G}\right)$. From the lemma we have $B\left(y, 3 \delta_{G}\right) \subset H$. Therefore the geodesic ray $\left[e, z^{\prime}\right)$ enters $H$. Since the complementary half-space $H_{-}(M)$ is convex, the ray $\left[e, z^{\prime}\right)$ remains in $H$; thus $z^{\prime} \in \partial H$. With (5.2) it follows that $B\left(z, C^{-1} a^{-L-9 \delta_{G}} r\right) \subset \partial H$.

5.2. Invariant subsets and parabolic subgroups. We study now the parabolic subgroups of $\Gamma$ in connection with the action of $\Gamma$ on its boundary.

Definition 5.4. Let $M$ be a wall of $G$ and let $F$ be a subset of $\partial \Gamma$. We say that $M$ cuts $F$ if $F$ meets both subsets $\partial H_{-}(M)$ and $\partial H_{+}(M)$.

Theorem 5.5. Let $F$ be a subset of $\partial \Gamma$ containing at least two distinct points. Assume that $F$ is invariant under each reflection whose wall cuts $F$, and let $P$ be the subgroup of $\Gamma$ generated by these reflections. Then $P$ is a parabolic subgroup of $\Gamma$, the closure of $F$ is the limit set of $P$, and $P$ is the stabilizer of $F$ in $\Gamma$. 
To prove the theorem we introduce the following notion which will also be useful in the sequel:

Definition 5.6. Let $F$ be a subset of $\partial \Gamma$. Assume it contains at least two distinct points. If $\bar{F} \neq \partial \Gamma$ the convex hull $\complement_{F}$ of $F$ is the intersection of all the half-spaces $H$ in $G$ such that $F$ belongs to the interior of $\partial H$ in $\partial \Gamma$. It is a convex subgraph of $G$. If $\bar{F}=\partial \Gamma$ we set $\ell_{F}=G$.

Lemma 5.7. The limit set $\partial \bigodot_{F}$ is the closure of $F$. In addition every wall of $G$ which intersects $\varphi_{F}$ cuts $F$.

Proof. Since $e_{F}$ contains every geodesic in $G$ with both endpoints in $F$, one has $F \subset \partial \mathscr{C}_{F}$, and thus $\bar{F} \subset \partial \mathscr{C}_{F}$. To prove the converse inclusion pick a point $z \in \partial \Gamma \backslash \bar{F}$. Since $\bar{F}$ is a closed set, Proposition 5.2 insures the existence of a wall $M$, with associated half-spaces $H_{+}$and $H_{-}$such that

$$
z \in\left(\partial H_{+} \backslash \partial M\right) \text { and } \partial H_{+} \subset(\partial \Gamma \backslash F)
$$

It follows that $z \notin \partial H_{-}$and that $F \subset\left(\partial \Gamma \backslash \partial H_{+}\right)=\operatorname{int}\left(\partial H_{-}\right)$, therefore $z \notin \partial e_{F}$.

Let $M$ be a wall intersecting $\ell_{F}$ and let $(x, y)$ be an edge in the intersection. Since $e_{F}$ is a closed subset of $G$ it contains $x$ and $y$. Hence both half-spaces $H_{+}, H_{-}$bounded by $M$ intersect $C_{F}$. It follows that $F$ is contained neither in $\operatorname{int}\left(\partial H_{+}\right)=\partial \Gamma \backslash \partial H_{-}$, nor $\operatorname{in} \operatorname{int}\left(\partial H_{-}\right)=\partial \Gamma \backslash \partial H_{+}$. Thus $M$ cuts $F$.

Proof of Theorem 5.5. Consider the convex hull $\ell_{F}$ of $F$ in $G$. The canonical action of $\Gamma$ on $G \cup \partial \Gamma$ possesses the following property:

$$
g\left(\mathcal{C}_{F}\right)=\boldsymbol{\ell}_{g(F)} \text { for all } g \in \Gamma \text {. }
$$

Therefore, up to a translation of $F$ by a group element and a conjugation of $P$, we may assume that $e$ belongs to $\ell_{F}$.

The graph $\ell_{F}$ is a closed non-empty $P$-invariant convex subset of $G$. At first we claim that $P$ acts simply transitively on it. Since $\Gamma$ acts freely on $G^{0}, P$ acts freely on $\ell_{F}^{0}$. To establish the transitivity, let $x \in \bigodot_{F}^{0}$ and let $x_{0}=e, x_{1}, \ldots, x_{n}=x$ be the successive vertices of a geodesic segment joining $e$ to $x$ in $G$. Since $\ell_{F}$ is convex, the edge $\left(x_{k}, x_{k+1}\right)$ belongs to $\ell_{F}$ and thus the wall $M_{k}$ between $x_{k}$ and $x_{k+1}$ intersects $e_{F}$. According to Lemma 5.7, it cuts $F$. Therefore the reflection along the wall $M_{k}$ belongs to $P$. In consequence every $x_{i}$ belongs to $P$. The transitivity follows.

The equality $P=\bigodot_{F}^{0}$ and Lemma 5.7 imply that $\partial P=\bar{F}$. Moreover the stabilizer of $F$ in $\Gamma$ stabilizes $\varphi_{F}^{0}$ too, so it coincides with $P$.

Finally we claim that $P$ is equal to the special subgroup $\Gamma_{I}$ where $I$ is the set of elements $s \in S$ such that the walls $M_{S}$ intersect $\ell_{F}$. The inclusion $\Gamma_{I}<P$ comes from Lemma 5.7. To establish the converse one, let $x \in P$. We shall prove 
by induction on $|x|$ that $x$ belongs to $\Gamma_{I}$. When $|x|=0$ this is obvious. Let $n \in \mathbb{N}^{*}$, assume that the elements $y \in P$ with $|y| \leq n-1$ belong in $\Gamma_{I}$, and consider $x \in P$ with $|x|=n$. Pick a geodesic segment $\gamma \subset G$ joining $e$ to $x$, and denote by $x_{0}=e, x_{1}, \ldots, x_{n}=x$ its successive vertices. Since $x \in \ell_{F}^{0}$, the convexity of $\ell_{F}$ implies that $\gamma$ is contained in $\mathcal{C}_{F}$. Therefore the reflection $x_{1}$ belongs to $\Gamma_{I}$. By the induction assumption we get that $x_{1}^{-1} x$ belongs to $\Gamma_{I}$, thus $x$ does too.

A first corollary concerns a special class of equivalence relations on $\partial \Gamma$. Examples of such equivalence relations will be considered in Section 10.

Corollary 5.8. Consider a $\Gamma$-invariant equivalence relation on $\partial \Gamma$ whose cosets are connected. Then:

(1) The closure of each coset is either a point or a parabolic limit set.

(2) If a nontrivial coset $F$ is path-connected, and $P$ is the parabolic subgroup with $\bar{F}=\partial P$, then for every $\epsilon>0$ and every path $\eta:[0,1] \rightarrow \partial P$, there is a path $\eta^{\prime}:[0,1] \rightarrow F$ such that

$$
d\left(\eta, \eta^{\prime}\right)=\max _{t \in[0,1]} d\left(\eta(t), \eta^{\prime}(t)\right)<\epsilon .
$$

Proof. (1) If $F$ is a coset, and a wall $M$ cuts $F$, then the limit set $\partial M$ intersects $F$, because $F$ is connected. Since the reflection in $M$ is the identity map on $\partial M$, the coset and its image intersect, so they are equal. Therefore the assertion follows from Theorem 5.5.

(2) Suppose $F$ is path-connected, $\bar{F}=\partial P, \eta \subset \partial P$ is a path, and $\epsilon>0$. By Proposition 5.2, we can find a finite collection of half-spaces $H_{1}, \ldots, H_{k} \subset G$ such that the limit sets $\partial H_{1}, \ldots, \partial H_{k} \subset \partial \Gamma$ each have diameter $<\epsilon$, and their interiors cover the image of $\eta$. We can then choose $0=t_{0}<t_{1}<\ldots<t_{n}=1$ such that for each $i$, the pair $\eta\left(t_{i-1}\right), \eta\left(t_{i}\right)$ is contained in $\partial H_{j_{i}}$ for some $j_{i} \in\{1, \ldots, n\}$, and $\operatorname{diam}\left(\eta\left(\left[t_{i-1}, t_{i}\right]\right)\right)<\epsilon / 2$. Since $F$ is dense in $\partial P$, for each $j \in\{1, \ldots, n\}$ we may choose $s_{j} \in F$ close enough to $\eta\left(t_{j}\right)$ such that for all $i$, the pair $s_{i-1}, s_{i}$ also lies in $\partial H_{j_{i}}$. By the path connectedness of $F$, we may join $s_{i-1}$ to $s_{i}$ by a path $\bar{\gamma}_{i} \subset F$; reflecting the portion of $\bar{\gamma}_{i}$ lying outside $\partial H_{j_{i}}$ into $\partial H_{j_{i}}$ using the reflection whose wall bounds $H_{j_{i}}$, we get a path $\gamma_{i} \subset \partial H_{j_{i}}$ joining $s_{i-1}$ to $s_{i}$. Concatenating the $\gamma_{i}$ 's, we obtain the desired path $\eta^{\prime}$.

The same proof as for part (1) of the previous corollary gives:

Corollary 5.9. Let $\Phi$ be a quasiconvex subgroup of $\Gamma$ with connected limit set. Assume that for all $g \in \Gamma$, the intersection $g(\partial \Phi) \cap \partial \Phi$ is either empty, or equal to $\partial \Phi$. Then $\Phi$ is virtually a parabolic subgroup of $\Gamma$. In other words there exists a parabolic subgroup $P$ of $\Gamma$ such that $\Phi \cap P$ is of finite index in both subgroups $\Phi$ and $P$. 
Corollary 5.10. Each connected component of $\partial \Gamma$ containing more than one point is a parabolic limit set.

5.3. Shadowing curves. In this paragraph we present a sort of a quantitative version of Theorem 5.5 .

Let $F$ be a subset in $\partial \Gamma$. As before we denote by $\ell_{F}$ its convex hull in $G$ (see Definition 5.6). The canonical action of $\Gamma$ on $G \cup \partial \Gamma$ possesses the following property:

$$
g\left(\boldsymbol{C}_{F}\right)=\boldsymbol{C}_{g(F)} \text { for all } g \in \Gamma .
$$

Therefore, up to a translation of $F$ by a group element, we are in the situation where $e_{F}$ contains $e$ the identity of $\Gamma$.

Definition 5.11. Let $\gamma$ be a non-constant curve in $\partial \Gamma$, let $I$ be a non-empty subset of $S$ and let $L \geq 0$. We say that $\gamma$ is a $(L, I)$-curve if $e \in \ell_{\gamma}$, and if for every $s \in I$ there exists an edge $a_{s}$ of type $s$ in $\ell_{\gamma}$ with $\operatorname{dist}\left(e, a_{s}\right) \leq L$.

Proposition 5.12. Let $\epsilon>0$ and let $L, I$ be as in the above definition. Let $P \leqq \Gamma$ be a conjugate of $\Gamma_{I}$, and let $\eta$ be a (parametrized) curve contained in $\partial P$. There exists a finite subset $E \subset \Gamma$ such that for any $(L, I)$-curve $\gamma$ the subset $\bigcup_{g \in E} g \gamma \subset \partial \Gamma$ contains a curve which approximates $\eta$ to within $\epsilon$ with respect to the $C^{0}$ distance.

Proof. Since $P=h \Gamma_{I} h^{-1}$ for some $h \in \Gamma$, we have $\partial P=h\left(\partial \Gamma_{I}\right)$. Moreover $h$ is a bi-Lipschitz homeomorphism of $\partial \Gamma$. Therefore it is enough to establish the proposition for $P=\Gamma_{I}$.

First step. We show that for every $(L, I)$-curve $\gamma$ the subset

$$
\bigcup_{\{g \in \Gamma ;|g| \leq L\}} g \gamma
$$

of $\partial \Gamma$ contains a curve passing through every $\partial M_{s}$ with $s \in I$. For this purpose pick $s \in I$, let $a_{s} \subset \mathcal{C}_{\gamma}$ be an edge of type $s$ with $\operatorname{dist}\left(e, a_{s}\right) \leq L$, and let $M$ be the wall containing $a_{s}$. Let $g \in H_{-}(M)$ be such that $a_{s}=(g, g s)$. The geodesic segment $[e, g]$ belongs to $\ell_{\gamma}$ (by convexity). Denote by

$$
g_{0}=e, g_{1}=\sigma_{1}, \quad g_{2}=\sigma_{1} \sigma_{2}, \ldots, \quad g_{n}=g=\sigma_{1} \ldots \sigma_{n},
$$

the successive vertices of the segment $[e, g]$ with $\sigma_{i} \in S$. According to Lemma 5.7 the wall $M_{i}$ passing between $g_{i}$ and $g_{i+1}$ cuts $\gamma$. Thus for every $i \in\{0, \ldots, n-1\}$ the curves $\gamma$ and $g_{i} \sigma_{i+1} g_{i}^{-1} \gamma$ intersect. One has $g_{i} \sigma_{i+1} g_{i}^{-1}=g_{i} g_{i+1}^{-1}$. It follows that the subset $\gamma \cup g_{1}^{-1} \gamma \cup \cdots \cup g_{n}^{-1} \gamma$ is an arcwise connected set. It intersects the limit set of the wall $g^{-1}(M)=M_{S}$. Thus the subset

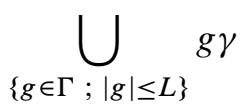


is an arcwise connected set which intersects the limit set of every wall $M_{s}$ with $s \in I$. Second step. Consider a collection $H_{1}, \ldots, H_{k}$ of half-spaces in $G$ all of them intersecting the special subgroup $\Gamma_{I}$ properly i.e. $\Gamma_{I} \cap H_{i}$ is distinct from $\emptyset$ and $\Gamma_{I}$. We will show that there exists a finite subset $E_{0}$ of $\Gamma$ such that for any $(L, I)$ curve $\gamma$, one can find in the subset $\bigcup_{g \in E_{0}} g \gamma \subset \partial \Gamma$ a curve passing through every $\partial H_{1}, \ldots, \partial H_{k}$. To do so, pick for each $i \in\{1, \ldots, k\}$ an element $p_{i} \in \Gamma_{I}$ adjacent to the wall which bounds $H_{i}$. Let $c$ be a path in $G$ which joins successively $p_{1}, \ldots, p_{k}$ and whose vertices $c_{1}, \ldots, c_{n}$ lie in $\Gamma_{I}$. Define

$$
E_{0}=\left\{c_{i} g \in \Gamma ;|g| \leq L, 1 \leq i \leq n\right\},
$$

and let $\theta$ be a curve made of translates of $\gamma$ passing through every $\partial M_{s}$ with $s \in I$ (as constructed in step 1). The subset $\bigcup_{1 \leq i \leq n} c_{i} \theta$ meets the limit set of any wall $c_{i}\left(M_{s}\right)$ with $i \in\{1, \ldots, n\}$ and $s \in I$. In particular it intersects $\partial H_{1}, \ldots, \partial H_{k}$. In addition this is an arcwise connected set. Indeed write $c_{i+1}=c_{i} s=\sigma c_{i}$, with $s \in I$ and $\sigma=c_{i} s c_{i}^{-1}$. Then $c_{i+1} \theta=\sigma c_{i} \theta$. The curve $\theta$ intersects $\partial M_{s}$, thus $c_{i} \theta$ intersects $c_{i}\left(\partial M_{s}\right)$. The intersection set is pointwise invariant by the reflection $\sigma$ and thus it belongs to $c_{i+1} \theta$ too.

Last step. We finally prove the proposition. By Proposition 5.2 there exists a collection of half-spaces $H_{1}^{\prime}, \ldots, H_{k+1}^{\prime}$ of $G$ such that the union of their limit sets is a neighborhood of $\eta$ contained in the $\epsilon / 2$-neighborhood of $\eta$. Reordering if necessary we may assume that the curve $\eta$ enters successively $\partial H_{1}^{\prime}, \ldots, \partial H_{k}^{\prime}$. Pick a collection of half-spaces $H_{1}, \ldots, H_{k}$ each of them intersecting $\Gamma_{I}$ properly, and such that for every $i \in\{1, \ldots, k\}$ one has

$$
\partial H_{i} \subset \partial H_{i}^{\prime} \cap \partial H_{i+1}^{\prime} .
$$

Existence of the $H_{i}$ 's follows from Proposition 5.2. According to step 2 there exists a subset $E_{0}$ of $\Gamma$ such that for every $(L, I)$-curve $\gamma$, one can find in the subset $\bigcup_{g \in E_{0}} g \gamma$ a curve passing through every $\partial H_{1}, \ldots, \partial H_{k}$. Denote by $\theta$ such a curve. The part of $\theta$ between $\partial H_{i-1}$ and $\partial H_{i}$ may exit from $\partial H_{i}^{\prime}$. If it happens reflect the outside part of $\theta$ along the wall which bounds $H_{i}^{\prime}$. The resulting curve can be parametrized to approximate $\eta$ to within $\epsilon$ with respect to the $C^{0}$ distance. Let $\sigma_{i}$ be the reflection along the wall of $H_{i}^{\prime}$. The following subset of $\Gamma$

$$
E=E_{0} \cup \bigcup_{i=2}^{k} \sigma_{i}\left(E_{0}\right)
$$

satisfies the property we were looking for.

We now establish the abundance of $(L, I)$-curves. Denote by $N_{r}(E)$ the open $r$-neighborhood in $\partial \Gamma$ of a subset $E \subset \partial \Gamma$. 
Proposition 5.13. Let $I \subset S$ and let $P \leqq \Gamma$ be a conjugate of $\Gamma_{I}$. For all $r>0$ there exist $L \geq 0$ and $\delta>0$ such that every curve $\gamma \subset \partial \Gamma$ satisfying the following conditions is an $(L, I)$-curve:

(i) Its convex hull contains $e$.

(ii) $\gamma \subset N_{\delta}(\partial P)$.

(iii) $\gamma \nsubseteq N_{r}(\partial Q)$ for any parabolic $Q \supsetneqq P$ with connected limit set.

Proof. Suppose by contradiction that for every $L \geq 0$ and $\delta>0$ there exists a curve $\gamma$ which satisfies properties (i), (ii) and (iii), and which is not an $(L, I)$-curve. Choose $L=n, \delta=1 / n$ and pick $\gamma_{n}$ a corresponding curve. We may assume, by extracting a subsequence if necessary, that there exists an element $s \in I$ such that for every $n \geq 1$ no edge of $\varrho_{\gamma_{n}} \cap B(e, n)$ is of type $s$. We can also suppose that the sequence of compact subsets $\left\{\gamma_{n}\right\}_{n \geq 1}$ converges with respect to the Hausdorff distance to a non-degenerate continuum $\mathscr{L} \subset \partial P$. Indeed this follows from the compactness of the set of continua in a compact metric space, equipped with the Hausdorff distance (see [Mun75], p. 281). In addition, by a standard diagonal argument, we may assume the sequence $\left\{\mathscr{C}_{\gamma_{n}}\right\}_{n \geq 1}$ converges to a convex subset $\mathcal{C} \subset G$ on every compact subset of $G$. With item (i) one has $e \in \mathcal{C}$. Moreover one sees easily that $\mathscr{L} \subset \partial \mathcal{C}$. The fact that no edge of $\mathscr{C}$ is of type $s$ implies that $\mathscr{L}$ is contained in the limit set of the special subgroup generated by $S \backslash\{s\}$. Intersections of parabolic subgroups are again parabolic subgroups (see [Dav08], Lemma 5.3.6); thus $\mathscr{L}$ is contained in the limit set of a proper parabolic subgroup of $P$. By Corollary 5.10 the connected component which contains $\mathscr{L}$ is the limit set of a proper parabolic subgroup $Q$ of $P$. So we get a contradiction with the hypothesis (iii).

Remarks and questions. 1) Theorem 5.5 admits a partial converse. Indeed let $P=g \Gamma_{I} g^{-1}$ be a parabolic subgroup of $\Gamma$ and let $M$ be a wall of $G$ such that $\partial P$ meets both open subsets $\partial H_{-}(M) \backslash \partial M$ and $\partial H_{+}(M) \backslash \partial M$. Then a convexity argument shows that $M$ admits an edge whose endpoints lie in $g \Gamma_{I}$. Thus letting $\sigma$ be the reflection along $M$, there exist $h, h^{\prime} \in \Gamma_{I}$ such that $\sigma g h=g h^{\prime}$. It follows that $\sigma=g h^{\prime} h^{-1} g^{-1} \in P$, and so $\partial P$ is $\sigma$-invariant.

2) Let $\Gamma$ be an arbitrary hyperbolic group. The limit set of the intersection of two quasiconvex subgroups is the intersection of their limit sets (see [Gro93], p. 164). Hence for any quasiconvex subgroup $\Phi \leqq \Gamma$ the following properties are equivalent:

- For every $g \in \Gamma, g \partial \Phi \cap \partial \Phi=\partial \Phi$ or $\emptyset$.

- For every $g \in \Gamma$, either $g \Phi g^{-1} \cap \Phi$ is finite or is of finite index in both subgroups $g \Phi g^{-1}$ and $\Phi$.

3) Given a subset $E \subset \partial \Gamma$ there exists a unique smallest parabolic limit set $\partial P$ containing $E$, moreover if $E$ is connected and non-reduced to a point then $\partial P$ is so. Indeed this follows from the fact that parabolic subgroups are stable by intersection (see [Dav08], Lemma 5.3.6), from the property of intersections of limit sets recalled in Remark 2 above, and from Corollary 5.10. 
4) Let $\Gamma$ be a hyperbolic group and consider a closed $\Gamma$-invariant equivalence relation on $\partial \Gamma$ whose cosets are continua. What one can say about such equivalence relations? In particular for which groups $\Gamma$ do the nontrivial cosets arise as the limit sets of a finite collection of conjugacy classes of quasiconvex subgroups?

Note that the quotient space of $\partial \Gamma$ by a $\Gamma$-invariant closed equivalence relation $\sim$ is a compact metrizable space on which $\Gamma$ acts as a convergence group, i.e. $\Gamma$ acts properly discontinuously on the set of triples of distinct points of $\partial \Gamma / \sim$ (see [Bow99]).

\section{Combinatorial modulus and Coxeter groups}

In this section we study the combinatorial modulus on boundaries of hyperbolic Coxeter groups. Consider a hyperbolic Coxeter group $\Gamma$ with connected boundary, and let $Z$ be the metric space $\partial \Gamma$ equipped with a self-similar metric (see Definition 3.2). We fix in the sequel some $\kappa$-approximation $\left\{G_{k}\right\}_{k \in \mathbb{N}}$ of $Z$.

Let $d_{0}$ be a fixed (small) positive constant. As before $\mathcal{F}_{0}$ denotes the set of curves $\gamma \subset Z$ with $\operatorname{diam}(\gamma) \geq d_{0}$. We wish to establish a kind of filtration of $\mathcal{F}_{0}$ by "elementary curve families" (see the discussion after Corollary 6.2). To this aim we introduce the following families of curves. Let $\partial P \subset Z$ be a parabolic limit set and let $\delta, r>0$. Denote by $\mathcal{F}_{\delta, r}(\partial P)$ the family of curves $\gamma$ in $Z$ satisfying the following conditions:

$-\gamma \subset N_{\delta}(\partial P)$ and diam $\gamma \geq d_{0}$.

- $\gamma \nsubseteq N_{r}(\partial Q)$ for any connected parabolic limit set $\partial Q \varsubsetneqq \partial P$.

Notice that for $\delta$ small enough there is only a finite number of $\partial P$ such that $\mathcal{F}_{\delta, r}(\partial P) \neq$ $\emptyset$ (see Proposition 5.1).

For $\epsilon>0$ and for any (parametrized) curve $\eta$ in $Z$, let $\mathcal{U}_{\epsilon}(\eta)$ be the set of curves whose $C^{0}$ distance to $\eta$ is smaller than $\epsilon$.

Theorem 6.1. There exists a positive increasing function $\delta_{0}$ on $(0,+\infty)$, that depends on $d_{0}$ only, and which possesses the following property. Let $p \geq 1, \epsilon, r>0$, let $\partial P \subset Z$ be a parabolic limit set and let $\eta$ be any curve in $\partial P$. There exists $C=C\left(p, \epsilon, \eta, r, d_{0}\right)>0$ such that for every $\delta \leq \delta_{0}(r)$ and for every $k \in \mathbb{N}$,

$$
\operatorname{Mod}_{p}\left(\mathcal{F}_{\delta, r}(\partial P), G_{k}\right) \leq C \operatorname{Mod}_{p}\left(\mathcal{U}_{\epsilon}(\eta), G_{k}\right)
$$

In addition when $p$ belongs to a compact subset of $[1,+\infty)$ the constant $C$ may be chosen independent of $p$.

In combination with Proposition 2.1.1, this leads to:

Corollary 6.2. Let $\eta \in \mathcal{F}_{0}$ and let $\partial P$ be the smallest parabolic limit set containing $\eta$. Let $r>0$ be small enough in order that $\eta \nsubseteq \bar{N}_{r}(\partial Q)$ for any parabolic limit set 
$\partial Q \varsubsetneqq \partial P$. Let $\delta \leq \delta_{0}(r)$ be as in the previous theorem. Let $\epsilon>0$ be small enough in order that $U_{\epsilon}(\eta) \subset \mathcal{F}_{\delta, r}(\partial P)$. Then for every $k \in \mathbb{N}$ one has

$$
\operatorname{Mod}_{p}\left(U_{\epsilon}(\eta), G_{k}\right) \leq \operatorname{Mod}_{p}\left(\mathcal{F}_{\delta, r}(\partial P), G_{k}\right) \leq C \operatorname{Mod}_{p}\left(u_{\epsilon}(\eta), G_{k}\right)
$$

where $C$ is the constant defined in the previous theorem.

Before going into the proof of the theorem, we discuss the meaning of these results and the roles and the interdependence of the parameters $r, \delta, \epsilon$. Corollary 6.2 means that for every curve $\eta \in \mathcal{F}_{0}$ a sufficiently small neighborhood $U_{\epsilon}(\eta)$ behaves - for the combinatorial modulus - like one of curve families $\mathcal{F}_{\delta, r}(\partial P)$ with $\delta \leq \delta_{0}(r)$. In particular the behaviour of $\operatorname{Mod}_{p}\left(U_{\epsilon}(\eta), G_{k}\right)$ is independent of $\epsilon$ (small enough depending on $\eta$ ). Theorem 6.1 states that for all $r>0$ and all $\delta \leq \delta_{0}(r)$ the modulus of $\mathcal{F}_{\delta, r}(\partial P)$ is controlled from above by the modulus of any neighborhood $U_{\epsilon}(\eta)$ of any curve $\eta \subset \partial P$. To complete the picture we notice that, given $r_{0}>0$, the curve family $\widetilde{F}_{0}$ can be express as a finite union of curve families $\widetilde{F}_{\delta_{i}, r_{i}}\left(\partial P_{i}\right)$ with $r_{i} \leq r_{0}$ and $\delta_{i} \leq \delta_{0}\left(r_{i}\right)$. To do so, one first defines the height of a connected parabolic limit set $\partial P$ to be the largest integer $n \geq 0$ such that there exists a chain of connected parabolic limit sets of the form

$$
\partial Q_{0} \varsubsetneqq \partial Q_{1} \varsubsetneqq \cdots \varsubsetneqq \partial Q_{n}=\partial P
$$

Then one considers the finite collection of the connected parabolic limit sets $\partial P_{i}$ of diameter larger than $\frac{d_{0}}{2}$, and defines the corresponding parameters $\left(r_{i}, \delta_{i}\right)$ by induction on the height of $\partial P_{i}$.

We now give the

Proof of Theorem 6.1. Instead of considering all curves with diameter larger than $d_{0}$, we may restrict ourselves to those whose convex hulls contain $e$ (see the discussion at the beginning of Subsection 5.3, and notice that $\left.\sup _{\gamma \in \mathcal{F}_{0}} \operatorname{dist}\left(e, \mathcal{C}_{\gamma}\right)<+\infty\right)$. In the sequel of the proof we make this restriction and we keep the same notation for the restriction of $\mathcal{F}_{\delta, r}(\partial P)$. We shall also use - without further mention - the metric equivalence between $Z$ and $\partial \Gamma$.

Let $I \subset S$ such that $P$ is a conjugate of $\Gamma_{I}$. Thanks to Proposition 5.13 there exists $L$ and $\delta>0$ depending only on $r$ such that every element of $\widetilde{F}_{\delta, r}(\partial P)$ is an $(L, I)$-curve. By Proposition 5.12 there exists a finite subset $E \subset \Gamma$ such that for every $\gamma \in \mathcal{F}_{\delta, r}(\partial P)$ the subset $\bigcup_{g \in E} g \gamma \subset Z$ contains a curve of $U_{\epsilon}(\eta)$. Let $G_{k}^{\prime}$ be the incidence graph of the covering of $Z$ by the elements of $\bigcup_{g \in E} g^{-1} G_{k}^{0}$. According to Proposition 2.2, and since $\Gamma$ acts on $Z$ by bi-Lipschitz homeomorphisms

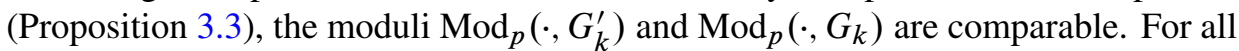
$\mathcal{U}_{\epsilon}(\eta)$-admissible function $\rho: G_{k}^{0} \rightarrow \mathbb{R}_{+}$define a function $\rho^{\prime}: G_{k}^{\prime 0} \rightarrow \mathbb{R}_{+}$by

$$
\rho^{\prime}\left(g^{-1} v\right)=\rho(v)
$$


where $v \in G_{k}^{0}$ and $g \in E$. We claim that $\rho^{\prime}$ is $\mathcal{F}_{8, r}(\partial P)$-admissible. Indeed let $\gamma \in \mathcal{F}_{\delta, r}(\partial P)$, and let $\theta \in \mathcal{U}_{\epsilon}(\eta)$ so that $\theta \subset \bigcup_{g \in E} g \gamma$. One has

$$
\ell_{\rho^{\prime}}(\gamma)=\sum_{g \in E} \ell_{\rho}(g \gamma) \geq \ell_{\rho}(\theta) \geq 1
$$

The claim follows. Thus

$$
\operatorname{Mod}_{p}\left(\mathscr{F}_{\delta, r}(\partial P), G_{k}^{\prime}\right) \leq|E| \sum_{v \in G_{k}^{0}} \rho(v)^{p},
$$

and so

$$
\operatorname{Mod}_{p}\left(\mathcal{F}_{\delta, r}(\partial P), G_{k}^{\prime}\right) \leq|E| \operatorname{Mod}_{p}\left(U_{\epsilon}(\eta), G_{k}\right) .
$$

We now present a companion result to Theorem 6.1. Its statement requires some notation. For a parabolic subgroup $P \leqq \Gamma$ let $\mathcal{N}_{r}(\partial P)$ be the family of curves $\gamma \subset N_{r}(\partial P)$ with diam $\gamma \geq d_{0}$. Let $\mathscr{L}$ be a collection of parabolic limit sets. For $r>0$ set

$$
\mathscr{F}_{r}(\mathscr{L}):=\mathscr{F}_{0} \backslash \bigcup_{\partial Q \in \mathscr{L}} \mathcal{N}_{r}(\partial Q) .
$$

We denote by Confdim $(\partial P)$ the Ahlfors regular conformal dimension of $\partial P$ (its definition is recalled in Remark 2 at the end of Section 3), and we set

$$
\operatorname{Confdim}(\mathscr{L}):=\max _{\partial Q \in \mathscr{L}} \operatorname{Confdim}(\partial Q) .
$$

The following property "controls" the modulus of neighborhoods of some limit sets by the modulus of the complementary subsets.

Theorem 6.3. Let $\mathscr{L}$ be a $\Gamma$-invariant collection of connected proper parabolic limit sets. Assume that there exists $\partial P \in \mathscr{L}$ such that for every $\partial Q \in \mathscr{L}$ with $\partial Q \neq \partial P$, the set $\partial P \cap \partial Q$ is totally disconnected or empty. Let $p>\operatorname{Confdim}(\partial P)$. There exist constants $C>0$ and $a \in(0,1)$ such that for $r>0$ small enough and for every $k \in \mathbb{N}$ one has

$$
\operatorname{Mod}_{p}\left(\mathcal{N}_{r}(\partial P), G_{k}\right) \leq C \cdot \sum_{\ell=0}^{k} \operatorname{Mod}_{p}\left(\mathcal{F}_{r}(\mathscr{L}), G_{k-\ell}\right) a^{\ell} .
$$

Corollary 6.4. Let $\mathscr{L}$ be a $\Gamma$-invariant collection of connected proper parabolic limit sets. Assume that for every $\partial P, \partial Q \in \mathscr{L}$ with $\partial Q \neq \partial P$, the set $\partial P \cap \partial Q$ is totally disconnected or empty. Let $p>\operatorname{Confdim}(\mathscr{L})$. There exist constants $C>0$ and $a \in(0,1)$ such that for $r>0$ small enough and for every $k \in \mathbb{N}$,

$$
\operatorname{Mod}_{p}\left(\mathcal{F}_{0}, G_{k}\right) \leq C \cdot \sum_{\ell=0}^{k} \operatorname{Mod}_{p}\left(\mathcal{F}_{r}(\mathscr{L}), G_{k-\ell}\right) a^{\ell} .
$$


Proof of Corollary 6.4. Notice that $\mathcal{F}_{0}=\mathcal{F}_{r}(\mathscr{L}) \cup \bigcup_{\partial P \in \mathscr{L}} \mathcal{N}_{r}(\partial P)$. Let $r>0$ be such that $d_{0}-2 r>0$. Only finitely many parabolic limit sets have diameter at least $d_{0}-2 r$ (see Proposition 5.1). Hence, all but a finite number of the curve families $\mathcal{N}_{r}(\partial P)$ are empty. Theorem 6.3 applied to the non-empty ones, in combination with the basic properties of the combinatorial modulus, completes the proof.

As an example the collection $\mathscr{L}$ of all circular limit sets satisfies the above hypotheses with $\operatorname{Confdim}(\mathscr{L})=1$.

The proof of the theorem relies on some lemmata. The first one shows that two connected parabolic limit sets whose intersection is at totally disconnected or empty, have to "move away one from another linearly".

Lemma 6.5. There is a constant $C_{0} \geq 1$ with the following property. Let $\partial P_{1}, \partial P_{2}$ be two connected parabolic limit sets such that either $\partial P_{1}=\partial P_{2}$ or $\partial P_{1} \cap \partial P_{2}$ is totally disconnected or empty. Assume that there exist $r>0$ and a curve $\gamma \subset$ $N_{r}\left(\partial P_{1}\right) \cap N_{r}\left(\partial P_{2}\right)$ with $\operatorname{diam}(\gamma) \geq C_{0} r$. Then $\partial P_{1}=\partial P_{2}$.

Proof. Applying the self-similarity property 3.1 we may assume that $\gamma$ has unit diameter. Then $\partial P_{1}$ and $\partial P_{2}$ have diameter bounded away from zero, and hence $P_{1}$ and $P_{2}$ belong to a finite collection of parabolic subgroups with connected limit sets (Proposition 5.1). But if $P_{1} \neq P_{2}$, then $\partial P_{1} \cap \partial P_{2}$ cannot contain an arc, because the intersection is totally disconnected. Hence by choosing $C_{0}$ large enough, the lemma follows.

From now on we consider a connected parabolic limit set $\partial P \subset Z$.

Lemma 6.6. Let $r>0, C \geq 1$ and let $\gamma \subset N_{r}(\partial P)$ be a curve with $\operatorname{diam}(\gamma)>C r$. For every $z \in(\gamma \backslash \partial P)$ there exists a subcurve $\gamma^{\prime}$ of $\gamma$ such that letting $d:=\operatorname{diam}\left(\gamma^{\prime}\right)$, one has

$$
\gamma^{\prime} \subset B(z, 4 d) \cap N_{d / C}(\partial P) \quad \text { and } \quad \gamma^{\prime} \nsubseteq N_{d /(8 C)}(\partial P)
$$

Proof. Either there exists a point $z_{1}$ on $\gamma$ with $d\left(z, z_{1}\right)<2 C \operatorname{dist}(z, \partial P)$ and $\operatorname{dist}\left(z_{1}, \partial P\right)=2 \operatorname{dist}(z, \partial P)$, or there does not.

If not then the part of $\gamma$ contained in the ball centered on $z$ of radius $2 C \operatorname{dist}(z, \partial P)$ lies in the $(2 \operatorname{dist}(z, \partial P))$-neighborhood of $\partial P$. The assumption on $\operatorname{diam}(\gamma)$ implies that $\gamma$ exits the ball $B(z, 2 C \operatorname{dist}(z, \partial P))$. Thus $\gamma$ contains a desired subcurve which moreover contains $z$.

If $z_{1}$ exists then we repeat the process with $z_{1}$ instead of $z$. Eventually we get a sequence of points $z_{0}, z_{1}, \ldots, z_{n}$ on $\gamma$ with $z_{0}=z$ such that for all indices $i$,

$$
\operatorname{dist}\left(z_{i}, \partial P\right)=2^{i} \operatorname{dist}(z, \partial P) \quad \text { and } \quad d\left(z_{i}, z_{i+1}\right)<2 C \operatorname{dist}\left(z_{i}, \partial P\right) .
$$

Since $\gamma \subset N_{r}(\partial P)$ and $\operatorname{dist}(z, \partial P)>0$ the process has to stop, let $z_{n}$ be the last point. From the first case argument we get a subcurve $\gamma^{\prime}$ of $\gamma$ such that letting $d=\operatorname{diam}\left(\gamma^{\prime}\right)$ 
we have

$$
z_{n} \in \gamma^{\prime} \subset B\left(z_{n}, 2 C \operatorname{dist}\left(z_{n}, \partial P\right)\right), d \geq 2 C \operatorname{dist}\left(z_{n}, \partial P\right),
$$

and

$$
\gamma^{\prime} \subset N_{d / C}(\partial P), \gamma^{\prime} \nsubseteq N_{d /(8 C)}(\partial P)
$$

Finally we compute

$$
d\left(z, z_{n}\right) \leq \sum_{i=0}^{n-1} d\left(z_{i}, z_{i+1}\right) \leq 2^{n+1} C \operatorname{dist}(z, \partial P)=2 C \operatorname{dist}\left(z_{n}, \partial P\right) \leq d .
$$

Therefore $\gamma^{\prime} \subset B(z, 4 d)$.

Pick for every $\ell \in \mathbb{N}$ a collection $\mathscr{B}_{\ell}$ of balls in $Z$ centered on $\partial P$ and of radius $2^{-\ell}$ such that the set $\left\{\frac{1}{2} B ; B \in \mathscr{B}_{\ell}\right\}$ is a minimal covering of $\partial P$. Let $\mathcal{B}=\bigcup_{\ell \in \mathbb{N}} \mathcal{B}_{\ell}$. Recall that the radius of a ball $B$ is denoted by $r(B)$. From the previous lemma we get:

Lemma 6.7. Let $r>0, C \geq 1$ and let $\gamma \subset N_{r}(\partial P)$ be a curve with $\operatorname{diam}(\gamma)>C r$. For every $z \in(\gamma \backslash \partial P)$ there exists a ball $B \in \mathcal{B}$ and a subcurve $\gamma^{\prime}$ of $\gamma$ such that letting $d=\operatorname{diam}\left(\gamma^{\prime}\right)$ we have

$$
\gamma^{\prime} \cup\{z\} \subset B, \quad r(B) \leq 36 d, \quad \gamma^{\prime} \subset N_{d / C}(\partial P) \quad \text { and } \quad \gamma^{\prime} \nsubseteq N_{d /(8 C)}(\partial P) .
$$

Proof. Consider the subcurve $\gamma^{\prime}$ obtained in the previous lemma. We compute

$$
\operatorname{dist}(z, \partial P) \leq 4 d+\operatorname{dist}\left(\gamma^{\prime}, \partial P\right) \leq 5 d .
$$

Let $w$ be a point in $\partial P$ which realises $\operatorname{dist}(z, \partial P)$, we have $\gamma^{\prime} \cup\{z\} \subset B(w, 9 d)$. Pick a ball $B \in \mathcal{B}$ such that $w \in \frac{1}{2} B$ and $r(B) / 4 \leq 9 d \leq r(B) / 2$. Then $B$ contains $B(w, 9 d)$ and hence it contains $\gamma^{\prime} \cup\{z\}$. In addition we have $r(B) \leq 36 d$.

Proof of Theorem 6.3. Let $\partial P \in \mathscr{L}$ be the connected proper limit set of the statement, and let $p>\operatorname{Confdim}(\partial P)$. For appropriate $r>0$ we wish to construct on every scale a $\mathcal{N}_{r}(\partial P)$-admissible function $\rho_{k}: G_{k}^{0} \rightarrow \mathbb{R}_{+}$with controlled $p$-mass. For this purpose we equip $\partial P$ with an Ahlfors regular metric $\delta_{\partial P}$, which is quasi-Möbius equivalent ${ }^{3}$ to the restriction of the $Z$-metric to $\partial P$, and whose Hausdorff dimension $Q$ satisfies $Q<p$. Let $\mu$ be the $Q$-Hausdorff measure of $\left(\partial P, \delta_{\partial P}\right)$. Given any ball $B \subset Z$ centered on $\partial P$, its trace on $\partial P$ is comparable to a ball of $\left(\partial P, \delta_{\partial P}\right)$. In other words there are balls $B_{1}^{\prime}, B_{2}^{\prime} \subset\left(\partial P, \delta_{\partial P}\right)$ of radii $\tau_{1}, \tau_{2}$ such that $B_{1}^{\prime} \subset(B \cap \partial P) \subset$ $B_{2}^{\prime}$, with $\frac{\tau_{2}}{\tau_{1}}$ smaller than an absolute constant. We denote by $\tau(B)$ the radius of a minimal ball of $\left(\partial P, \delta_{\partial P}\right)$ containing $B \cap \partial P$. One has

$$
\mu(B \cap \partial P) \asymp \tau(B)^{Q} .
$$

\footnotetext{
${ }^{3}$ Two metrics $d_{1}, d_{2}$ on a space $X$ are said to be quasi-Möbius equivalent if the identity map $\left(X, d_{1}\right) \rightarrow$ $\left(X, d_{2}\right)$ is a quasi-Möbius homeomorphism.
} 
Let $\mathcal{B}$ be a ball family as considered in the statement of the previous lemma. We inflate every element $B \in \bigcup_{\ell=0}^{k} \mathscr{B}_{\ell}$ to essentially unit diameter as in Definition 3.1. Let $g_{B}$ be the corresponding group element. Define $G_{k} \cap B$ to be the incidence graph of the covering of $B$ by the subsets $v \in G_{k}^{0}$ with $v \cap B \neq \emptyset$. Note that for $B \in \mathscr{B}_{\ell}$, the graph $G_{k} \cap B$ may be considered as a $\left(2 \kappa L_{0}\right)$-approximation of $g_{B}(B)$ on scale $k-\ell$ (via the group element $g_{B}$ ).

For $k \in \mathbb{N}$ and $r>0$ write $m_{k}:=\operatorname{Mod}_{p}\left(\mathcal{F}_{r}(\mathscr{L}), G_{k}\right)$ for simplicity. Suppose that $r$ is small compared to $L_{0}, C_{0}, d_{0}\left(L_{0}\right.$ and $C_{0}$ are defined respectively in statements 3.1 and 6.5). Then using the group element $g_{B}$ and the $\Gamma$-invariance of $\mathscr{L}$, we may pull back to $G_{k} \cap B$ a normalized minimal $\mathcal{F}_{r}(\mathscr{L})$-admissible function on scale $k-\ell$, in order to get for every ball $B \in \bigcup_{\ell=0}^{k} \mathcal{B}_{\ell}$ a function $\rho_{B}:\left(G_{k} \cap B\right)^{0} \rightarrow \mathbb{R}_{+}$, with the following properties:

(i) Its $p$-mass satisfies, with $D \geq 1$ independent of $k$ and of $B$,

$$
M_{p}\left(\rho_{B}\right) \leq D \cdot m_{k-\ell} \cdot \tau(B)^{p}
$$

(ii) Every curve $\gamma \subset B$ whose diameter is larger than $r(B) / 36$ picks up a $\left(\rho_{B}\right)$ length larger than $\tau(B)$, unless it lies in the $\left(10^{-3} C_{0}^{-1} r(B)\right)$-neighborhood of a limit set of $\mathscr{L}$.

Define $\rho_{k}$ by

$$
\rho_{k}(v)=\max \rho_{B}(v) \text { for all } v \in G_{k}^{0},
$$

where the maximum is over all $B \in \bigcup_{\ell=0}^{k} \mathscr{B}_{\ell}$ with $v \cap B \neq \emptyset$. Its $p$-mass is linearly bounded from above by

$$
\sum_{\ell=0}^{k} \sum_{B \in \mathcal{B}_{\ell}} M_{p}\left(\rho_{B}\right)
$$

Since the metric $\delta_{\partial P}$ and the restriction of the $Z$-metric are quasi-Möbius equivalent, they are Hölder equivalent (see [Hei01]). In particular there is $\alpha>0$ such that for every $B \in \mathcal{B}_{\ell}$ one has $\tau(B) \lesssim 2^{-\alpha \ell}$. With item (i) one obtains that the $p$-mass of $\rho_{k}$ is linearly bounded from above by

$$
\sum_{\ell=0}^{k} m_{k-\ell} \sum_{B \in B_{\ell}} \tau(B)^{p} \leq \sum_{\ell=0}^{k} m_{k-\ell} \sum_{B \in B_{\ell}} \tau(B)^{Q} a^{\ell} \asymp \mu(\partial P) \sum_{\ell=0}^{k} m_{k-\ell} a^{\ell},
$$

with $a=2^{-\alpha(p-Q)}$. Because $p>Q$ one has $0<a<1$.

It remains to prove that $\rho_{k}$ is a $\mathcal{N}_{r}(\partial P)$-admissible function up to a multiplicative constant independent of $k$. Let $\gamma \in \mathcal{N}_{r}(\partial P)$.

Claim: For every $z \in \gamma$ there exists a ball $B_{z} \in \bigcup_{\ell=0}^{k} \mathscr{B}_{\ell}$ with $z \in B_{z}$ and such that the $\rho_{k}$-length of $\gamma \cap B_{z}$ is larger than $\tau\left(B_{z}\right)$. 
Indeed, assume at first that $z \in \gamma \cap \partial P$. Pick $B_{z} \in \mathscr{B}_{k}$ with $z \in B_{z}$. Since for $B \in \mathscr{B}_{k}$ we may assume that the function $\rho_{B}$ is larger than $\tau(B)$, the ball $B_{z}$ admits the desired property.

Now assume that $z \in \gamma \backslash \partial P$. Because $r$ is small compared with $d_{0}$ the hypotheses of Lemma 6.7 are satisfied with $C=C_{0}$. Let $B \in \mathcal{B}$ as in this lemma. If $B$ belongs to $\bigcup_{\ell \geq k} \mathscr{B}_{\ell}$, then choosing $B_{z} \in \mathscr{B}_{k}$ with $B \subset B_{z}$ we are back to the previous case.

If $B \in \bigcup_{\ell=0}^{k-1} \mathcal{B}_{\ell}$, let $B_{z}=B$ and consider the subcurve $\gamma^{\prime}$ given by Lemma 6.7 applied with $C=C_{0}$. It lies in the $\left(d / C_{0}\right)$-neighborhood of $\partial P$, hence our hypotheses and Lemma 6.5 assert that there is no other limit set in $\mathscr{L}$ whose $\left(d / C_{0}\right)$ neighborhood contains $\gamma^{\prime}$. In addition we have

$$
10^{-3} C_{0}^{-1} r\left(B_{z}\right) \leq 10^{-3} C_{0}^{-1} 36 d \leq d /\left(8 C_{0}\right),
$$

thus $\gamma^{\prime}$ does not lie in any $\left(10^{-3} C_{0}^{-1} r\left(B_{z}\right)\right)$-neighborhood of a limit set of $\mathscr{L}$. Therefore the claim follows from item (ii).

According to $5 r$-covering theorem (see [Mat95]) we may extract from the collection $\left\{10 B_{z} ; z \in \gamma\right\}$ a finite cover $\left\{10 B_{1}, \ldots, 10 B_{n}\right\}$ of $\gamma$ such that the balls $2 B_{1}, \ldots, 2 B_{n}$ are pairwise disjoint. This last property ensures that for every $v \in G_{k}^{0}$ the number of elements of $\left\{B_{1}, \ldots, B_{m}\right\}$ which meet $v$ is bounded from above by a constant $C_{1}$ which depends only on $\kappa$ and on the geometry of $Z$. With the claim we obtain

$$
C_{1} L_{\rho_{k}}(\gamma) \geq \sum_{i=1}^{n} L_{\rho_{k}}\left(\gamma \cap B_{i}\right) \geq \sum_{i=1}^{n} \tau\left(B_{i}\right) .
$$

Reordering if necessary we may assume that $\gamma$ enters successively the $10 B_{i}$ 's. Let $w_{i} \in \partial P$ be the center of $B_{i}$. For every $i \in\{1, \ldots, n-1\}$ the balls $10 B_{i}$ and $10 B_{i+1}$ intersect, so one of two balls $20 B_{i}$ and $20 B_{i+1}$ contains the pair $\left\{w_{i}, w_{i+1}\right\}$. Thus $20 B_{i} \cap \partial P$ and $20 B_{i+1} \cap \partial P$ intersect. Since the metric $\delta_{\partial P}$ and the restriction of the $Z$-metric are quasi-Möbius, it follows from the triangle inequality that the last sum is linearly bounded from below by the $\delta_{\partial P}$-diameter of $\bigcup_{i=1}^{n} 20 B_{i} \cap \partial P$. Therefore $L_{\rho_{k}}(\gamma)$ is bounded from below in terms of $d_{0}$.

\section{Cannon Coxeter groups}

Using the techniques developed in the previous section we prove a special case of the Cannon's conjecture (Theorem 7.1). This result was already known, we review a proof due to M. Davis at the end of the section. However our methods are new and of different flavour. An application to the hyperbolic Coxeter groups whose boundaries are homeomorphic to the Sierpinski carpet is presented in Corollary 7.5 (the definition of the Sierpinski carpet is recalled in Section 4). 
Theorem 7.1. Let $\Gamma$ be a hyperbolic Coxeter group whose boundary is homeomorphic to the 2-sphere. Then there is a properly discontinuous, cocompact, and isometric action of $\Gamma$ on $\mathbb{H}^{3}$, the real hyperbolic space.

Assume $\Gamma$ is a hyperbolic Coxeter group whose boundary is homeomorphic to the 2-sphere. Let $\left\{G_{k}\right\}_{k \in \mathbb{N}}$ be a $\kappa$-approximation of $\partial \Gamma$, and let $d_{0}$ be a fixed small positive constant. As before we denote by $\mathcal{F}_{0}$ the family of curves $\gamma \subset \partial \Gamma$ with $\operatorname{diam} \gamma \geq d_{0}$. The following proposition is the main step of the proof.

Proposition 7.2. For $p=2$ the modulus $\operatorname{Mod}_{p}\left(\widetilde{F}_{0}, G_{k}\right)$ is bounded independently of the scale $k$.

Its proof will use the following general dichotomy:

Lemma 7.3. Let $\Phi$ be a hyperbolic group whose boundary is connected. Then either $\partial \Phi$ is a topological circle, or it contains a subset homeomorphic to the capital letter $\mathrm{H}$.

Proof. Since $\partial \Phi$ is locally connected and nontrivial, it contains an arc $X$ (see [BK05b]). If $x \in X$ is not an endpoint and if some neighborhood of $x$ in $\partial \Phi$ is contained in $X$, then - since the action of $\Phi$ on $\partial \Phi$ is minimal $-\partial \Phi$ is a 1-manifold and so is homeomorphic to the circle. Otherwise, if $x_{1}, x_{2} \in X$ are distinct points which are not endpoints of $X$, then we may find $y_{1}, y_{2} \in \partial \Phi \backslash X$ and disjoint arcs $J_{i} \subset \partial \Phi(i=1,2)$, joining $y_{i}$ to $x_{i}$. Trimming the $J_{i}$ 's and taking the union $X \cup J_{1} \cup J_{2}$, we get a subset homeomorphic to the capital letter $H$, provided $y_{i}$ is sufficiently close to $x_{i}$.

Proof of Proposition 7.2. With the notation of Section 6, let $\mathscr{L}$ be the collection of

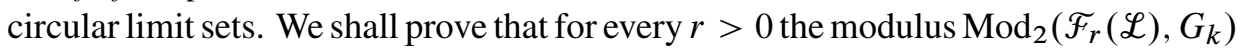
is bounded independently of the scale $k$. Proposition 7.2 will then follow from Corollary 6.4. To establish the desired bound, we notice that $\mathcal{F}_{r}(\mathscr{L})$ decomposes as a finite union of curve families $\mathcal{F}_{\delta_{i}}, r_{i}\left(\partial P_{i}\right)$, with $\partial P_{i}$ non-circular connected parabolic limit set and $\delta_{i} \leq \delta_{0}\left(r_{i}\right)$ - see the discussion that follows Corollary 6.2. Therefore it is enough to show that for every $r>0, \delta \leq \delta_{0}(r)$, and every non-circular connected parabolic limit set $\partial P \subset \partial \Gamma$, the $\operatorname{modulus} \operatorname{Mod}_{2}\left(\mathcal{F}_{\delta, r}(\partial P), G_{k}\right)$ is bounded independently of the scale $k$.

Let $\partial P$ be a non-circular connected parabolic limit set. According to the above lemma, $\partial P$ contains a graph homeomorphic to the capital letter $H$. Express the capital letter $\mathrm{H}$ as the union of two $\operatorname{arcs} \alpha, \alpha^{\prime}$ where $\alpha$ joins the upper left endpoint to the lower right endpoint, and $\alpha^{\prime}$ joins the upper right endpoint to the lower left endpoint. Bending the two vertical segments of $\alpha^{\prime}$ we obtain a horizontal segment denoted by $\beta$. Consider a thin horizontal rectangle $\mathcal{R}$ which is a small planar neighborhood of the horizontal segment $\alpha \cap \beta$. Say that a planar curve $\gamma$ cross-connects in $\mathcal{R}$ two opposite sides of $\mathcal{R}$, if $\gamma$ admits a subcurve contained in $\mathcal{R}$ and joining these sides. Then every curve which lies within sufficiently small $C^{0}$ distance from $\alpha$ cross-connects in $\mathcal{R}$ 
its horizontal sides, and every curve which lies within sufficiently small $C^{0}$ distance from $\beta$ cross-connects in $\mathcal{R}$ its vertical sides.

By assumption a similar picture appears in $\partial \Gamma$ and $\partial P$ too: there exist two arcs $\eta_{1}, \eta_{2}$ in $\partial P$ and there exists a topological rectangle $\mathcal{R} \subset \partial \Gamma$ such that every curve in $\partial \Gamma$ which lies within sufficiently small $C^{0}$ distance from $\eta_{1}$ cross-connects in $\mathcal{R}$ its horizontal sides, and every curve which lies within sufficiently small $C^{0}$ distance from $\eta_{2}$ cross-connects in $\mathcal{R}$ its vertical sides. Let $\mathscr{F}_{h}(\mathcal{R})$ (resp. $\mathscr{F}_{v}(\mathcal{R})$ ) be the family of curves contained in $\mathcal{R}$ and joining its horizontal (resp. vertical) sides. With Proposition 2.1 we get that, for $\epsilon>0$ small enough,

$$
\operatorname{Mod}_{2}\left(U_{\epsilon}\left(\eta_{1}\right), G_{k}\right) \leq \operatorname{Mod}_{2}\left(\mathscr{F}_{h}(\mathcal{R}), G_{k}\right),
$$

and

$$
\operatorname{Mod}_{2}\left(U_{\epsilon}\left(\eta_{2}\right), G_{k}\right) \leq \operatorname{Mod}_{2}\left(\mathscr{F}_{v}(\mathcal{R}), G_{k}\right) .
$$

The next lemma shows that $\min _{i=1,2} \operatorname{Mod}_{2}\left(U_{\epsilon}\left(\eta_{i}\right), G_{k}\right)$ is bounded independently of $k$. Therefore Theorem 6.1 applied to $\eta_{1}$ or $\eta_{2}$ shows that $\operatorname{Mod}_{2}\left(\mathcal{F}_{\delta, r}(\partial P), G_{k}\right)$ is bounded independently of the scale $k$.

Lemma 7.4. There exists a constant $C \geq 1$ such that for any topological rectangle $\mathcal{R} \subset \partial \Gamma$ one has, for every $k \in \mathbb{N}$ large enough,

$$
\operatorname{Mod}_{2}\left(\mathscr{F}_{h}(\mathcal{R}), G_{k}\right) \cdot \operatorname{Mod}_{2}\left(\mathscr{F}_{v}(\mathcal{R}), G_{k}\right) \leq C .
$$

Proof. A short and straightforward proof for annuli instead of rectangles can be found in [Hai09] (proof of lemme 2.14). This proof applies verbatim to rectangles too.

We now sketch a less direct but enlightening proof. Let $\bmod (\cdot)$ denote the classical analytic modulus on the Euclidean sphere $S^{2}$. A well-known result asserts that for $\mathcal{R} \subset S^{2}$,

$$
\bmod \left(\mathcal{F}_{v}(\mathcal{R})\right) \cdot \bmod \left(\mathcal{F}_{h}(\mathcal{R})\right)=1 .
$$

The lemma follows from this fact and from some rather elaborate process to relate the combinatorial 2-modulus on $\partial \Gamma$ with the analytic modulus on $S^{2}$ (see [CFP99], Theorem 1.5, or [BK02], Corollary 8.8, for more details).

Proof of Theorem 7.1. According to Proposition 7.2 and Corollary 3.5 one obtains that $\partial \Gamma$ is quasi-Möbius homeomorphic to the Euclidean 2-sphere. As explained in the sketch of proof in Subsection 1.3, a theorem of Sullivan completes the proof ([Sul81], p. 468, see also [Tuk86]).

Corollary 7.5. Let $\Gamma$ be a hyperbolic Coxeter group whose boundary is homeomorphic to the Sierpinski carpet, then $\Gamma$ acts properly discontinuously by isometries on $\mathbb{H}^{3}$, and cocompactly on the convex-hull of its limit set.

Proof. A nonseparating topological circle in $\partial \Gamma$ is called a peripheral circle. A peripheral subgroup of $\Gamma$ is the stabilizer of a peripheral circle. In [KaK00], Theorem 5, the following results are proved. 
(i) There is only a finite number of conjugacy classes of peripheral subgroups.

(ii) Let $H_{1}, \ldots, H_{k}$ be a set of representatives of these classes, then the double group $\Gamma \star H_{i} \Gamma$ is a hyperbolic group with 2 -sphere boundary. ${ }^{4}$

Observe that Theorem 5.5 shows that every peripheral subgroup is a parabolic subgroup. Choose subsets $I_{1}, \ldots, I_{k}$ of $S$ such that the subgroups $P_{i}$ generated by $I_{i}$ form a set of representatives of conjugacy classes of peripheral subgroups. Then $\widehat{\Gamma}:=\Gamma \star P_{i} \Gamma$ is obviously an index 2 subgroup of a Coxeter group. Therefore the corollary follows from item (ii) and Theorem 7.1.

Another proof of Theorem 7.1. The following proof of Theorem 7.1 has been communicated to us by M. Davis. A theorem of Bestvina-Mess [BM91] and the boundary hypothesis show that $\Gamma$ is a virtual 3-dimensional Poincaré duality group. Then Theorem 10.9.2 of [Dav08] implies that $\Gamma$ decomposes as $\Gamma=\Gamma_{0} \times \Gamma_{1}$, where $\Gamma_{0}$ is a finite Coxeter group and where $\Gamma_{1}$ is a Coxeter group whose nerve is a 2sphere. Applying Andreev's theorem to the dual polyhedron to the nerve, one obtains that $\Gamma_{1}$ acts on $\mathbb{H}^{3}$ as a cocompact reflection group.

\section{The combinatorial Loewner property for Coxeter groups}

This section gives a sufficient condition for the boundary of a hyperbolic Coxeter group to satisfy the combinatorial Loewner property (Theorem 8.6). Some examples of groups for which the condition applies are presented in the next section.

8.1. Generic curves. Let $\Gamma$ be a hyperbolic Coxeter group whose boundary is connected. Let $Z$ be the metric space $\partial \Gamma$ equipped with a self-similar metric (see Definition 3.2). We fix in the sequel some $\kappa$-approximation $\left\{G_{k}\right\}_{k \in \mathbb{N}}$ of $Z$.

As before $d_{0}$ is a fixed small positive constant. For $r>0$ consider the family of curves $\gamma \subset Z$ with $\operatorname{diam}(\gamma) \geq d_{0}$, and such that $\gamma \nsubseteq N_{r}(\partial Q)$ for any connected proper parabolic limit set $\partial Q \subset Z$. It is a non-empty curve family provided $r$ is small enough. According to Corollary 6.2 its combinatorial modulus is comparable to the one of any of its subsets of the form $U_{\epsilon}(\eta)$. In particular it does not depend on $r$ up to a multiplicative constant independent of the scale. We shall denote such a family of curves by $\mathcal{F}^{g}$ and we shall call it a family of generic curves. Similarly we will call its combinatorial modulus the combinatorial modulus of generic curves.

When $p \geq 1$ is fixed we denote $\operatorname{Mod}_{p}\left(\mathcal{F}^{g}, G_{k}\right)$ by $m_{k}$ for simplicity. It will be also convenient to set $L_{k}:=m_{k}^{-1 / p}$. It satisfies

$$
L_{k}=\sup _{\rho} \frac{L_{\rho}\left(\mathcal{F}^{g}\right)}{M_{p}(\rho)^{1 / p}} \quad \text { with } L_{\rho}\left(\mathcal{F}^{g}\right):=\inf _{\gamma \in \mathcal{F}^{g}} L_{\rho}(\gamma) \text {, }
$$

\footnotetext{
${ }^{4} \Gamma \star_{H_{i}} \Gamma$ denotes the fundamental group of a graph of groups with two vertices and $k$ edges between them. The vertices groups are copies of $\Gamma$ and the edges groups are the subgroups $H_{i}$.
} 
where the supremum is over all positive functions of $G_{k}^{0}$.

We now study the asymptotic behaviour of the sequence $\left\{L_{k}\right\}_{k \in \mathbb{N}}$ depending on $p \geq 1$. Our main result establishes a weak type submultiplicative inequality for the sequence $\left\{L_{k}\right\}_{k \in \mathbb{N}}$ (Proposition 8.3). The results of this paragraph must be compared with those of Paragraph 3 concerning the family $\widetilde{F}_{0}$.

For the rest of the paragraph $p$ is an arbitrary number in $[1,+\infty)$. In the statement of the following two lemmata, $A_{0}$ denotes a fixed (large) positive number.

Lemma 8.1. There exist a constant $\Lambda \geq 1$ and a positive function $\Phi$ on $(0,+\infty)$ with the following properties. Let $B$ be a ball in $Z$ and let $k \in \mathbb{N}$ such that the radius $r(B)$ satisfies

$$
A_{0}^{-1} \leq \frac{r(B)}{2^{-k}} \leq A_{0}
$$

Consider two balls of same radius $B_{1}, B_{2} \subset B$, and let $t:=\frac{r\left(B_{i}\right)}{r(B)}$. Then for every

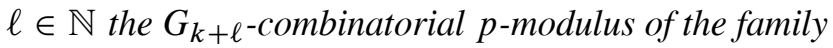

$$
\left\{\gamma \in \mathcal{F}\left(B_{1}, B_{2}\right) ; \gamma \subset \Lambda B\right\}
$$

is greater than $m_{\ell} \cdot \Phi(t)$.

Proof. Using the self-similarity property 3.1 we can restrict ourself to the case $k=0$. For every $0<r \leq \operatorname{diam} Z$ pick a maximal $\frac{r}{2}$-separated subset $E_{r} \subset Z$. Since $Z$ is linearly connected there exists a constant $\lambda>0$ such that every pair of points $x, y \in Z$ can be joined by a path of diameter less than $\lambda d(x, y)$. For every pair of points $x, y \in E_{r}$ we choose such a path and we call it $\eta_{x, y}$.

Let $\Lambda$ be a large number compared with $\lambda$. Consider any two $r$-balls $B_{1}, B_{2}$ contained in $B$. Pick two points $z_{1}, z_{2} \in E_{r}$ such that $B\left(z_{i}, r / 2\right) \subset B_{i}$ for $i=1,2$. Then with the notation of Section 6 we have

$$
U_{r / 2}\left(\eta_{z_{1}, z_{2}}\right) \subset\left\{\gamma \in \mathcal{F}\left(B_{1}, B_{2}\right) ; \gamma \subset \Lambda B\right\} .
$$

Given $r>0$ there is only a finite number of left-hand side terms. Therefore the desired inequality follows from Theorem 6.1 and from the fact that $r \asymp t$ by the rescaling assumption.

Lemma 8.2. There exist constants $\Lambda, D \geq 1$ and $b \in(0,1)$ with the following properties. Let $B$ be a ball in $Z$ and let $k \in \mathbb{N}$ be such that the radius $r(B)$ satisfies

$$
A_{0}^{-1} \leq \frac{r(B)}{2^{-k}} \leq A_{0} .
$$

Consider two continua $F_{1}, F_{2} \subset Z$ with $F_{i} \cap \frac{1}{4} B \neq \emptyset$ and $F_{i} \backslash B \neq \emptyset$. Then for every $\ell \in \mathbb{N}$ and every positive function $\rho$ on $G_{k+\ell}^{0}$ there exists a path in $\Lambda B$ joining $F_{1}$ to $F_{2}$ whose $\rho$-length is smaller than

$$
D \cdot M_{p}(\rho)^{1 / p} \cdot \sum_{n=0}^{\ell} L_{\ell-n} b^{n} .
$$


Proof. The arguments are basically the same as those used in the proof of Proposition 2.9. Indeed pick $q \in \mathbb{N}$ such that $\lambda:=2^{-q}$ and $a:=2 \cdot(8 \lambda)^{1 / p}$ satisfy $\lambda<1 / 8$ and $a<1$. Let $\Lambda$ be as in Lemma 8.1. Using Lemma 8.1 we can construct - like in the proof of Proposition $2.9-$ a path joining $F_{1}$ to $F_{2}$ in $\Lambda B$ whose $\rho$-length is smaller than

$$
D \cdot M_{p}(\rho)^{1 / p} \cdot \sum_{n=0}^{[\ell / q]} L_{\ell-n q} a^{n}
$$

where $D>0$ depends only on $\Lambda, q, p, \kappa$ and the geometry of $Z$, and where [.] denotes the integer part. Letting $b:=a^{1 / q}$ the lemma follows.

With the above two lemmata we obtain:

Proposition 8.3. There exist constants $b \in(0,1)$ and $C \geq 1$ such that for every pair of integers $k$, $\ell$ one has

$$
L_{k+\ell} \leq C \cdot L_{k} \cdot \sum_{n=0}^{\ell} L_{\ell-n} b^{n} .
$$

Moreover when $p$ belongs to a compact subset of $[1,+\infty)$ the constants $C$ and $b$ may be chosen independent of $p$.

Proof. Given a positive function $\rho_{k+\ell}$ of $G_{k+\ell}^{0}$ we wish to construct a curve $\gamma \in \mathcal{F}^{g}$ with controlled $\rho_{k+\ell}$-length. For this purpose we pick two disjoint balls $E_{1}, E_{2} \subset Z$ such that $\mathcal{F}\left(E_{1}, E_{2}\right) \subset \mathcal{F}^{g}$, and we will look for $\gamma$ in $\mathcal{F}\left(E_{1}, E_{2}\right)$.

For any $v \in G_{k}^{0}$, let $B_{v}$ be a ball containing $v$ and whose radius is approximately $2^{-k}$. Let $\rho_{k}: G_{k}^{0} \rightarrow \mathbb{R}_{+}$be defined by

$$
\rho_{k}(v)^{p}=\sum_{w \cap 4 \Lambda B_{v} \neq \emptyset} \rho_{k+\ell}(w)^{p},
$$

where $\Lambda$ is the constant appearing in Lemma 8.2. Since $Z$ is a doubling metric space one has

$$
M_{p}\left(\rho_{k+\ell}\right) \asymp M_{p}\left(\rho_{k}\right) .
$$

From Theorem 6.1 there exists a curve $\delta \in \mathcal{F}\left(E_{1}, E_{2}\right)$ whose $\rho_{k}$-length is linearly bounded from above by

$$
M_{p}\left(\rho_{k}\right)^{1 / p} \cdot L_{k}
$$

Let $v_{i} \in G_{k}^{0}$ so that $\delta$ enters successively $v_{1}, \ldots, v_{N}$, and set $B_{i}:=B_{v_{i}}$ for simplicity. Then, providing $2^{-k}$ is small enough compared to diam $E_{i}$, Lemma 8.2 allows one to construct by induction on $s \in\{1, \ldots, N\}$ a curve $\gamma_{s} \subset \bigcup_{i=1}^{s} 4 \Lambda B_{i}$, joining $E_{1}$ to $\bigcup_{i=s+1}^{N} B_{i} \cup E_{2}$, whose $\rho_{k+\ell}$-length is bounded linearly from above by

$$
\sum_{i=1}^{s} \rho_{k}\left(v_{i}\right) \cdot \sum_{n=0}^{\ell} L_{\ell-n} b^{n} .
$$


Therefore $\gamma:=\gamma_{N}$ belongs to $\mathcal{F}\left(E_{1}, E_{2}\right)$ and its $\rho_{k+\ell}$-length is bounded linearly from above by $L_{\rho_{k}}(\delta) \cdot \sum_{n=0}^{\ell} L_{\ell-n} b^{n}$. Thanks to estimates (8.1) and (8.2), the proposition follows.

Associated to generic curves, we introduce the following critical exponent:

$$
Q_{m}:=\sup \left\{p \in[1,+\infty) ; \lim _{k \rightarrow+\infty} \operatorname{Mod}_{p}\left(\mathcal{F}^{g}, G_{k}\right)=+\infty\right\} .
$$

The previous theorem combined with the monotonicity and the continuity of the function $p \mapsto \operatorname{Mod}_{p}\left(\mathcal{F}^{g}, G_{k}\right)$ gives

Corollary 8.4. The set of $p \geq 1$ such that $\lim _{k \rightarrow+\infty} \operatorname{Mod}_{p}\left(\mathscr{F}^{g}, G_{k}\right)=+\infty$ is equal to the interval $\left[1, Q_{m}\right)$, in particular it is void if $Q_{m}=1$.

Proof. As a consequence of the weak submultiplicative inequality stated in Proposition 8.3 one has

$$
\lim _{k \rightarrow+\infty} L_{k}=0 \Longleftrightarrow \text { there exists } \ell \in \mathbb{N} \text { with } \sum_{n=0}^{\ell} L_{\ell-n} b^{n}<C^{-1},
$$

where $C$ is the constant appearing in the weak submultiplicative inequality. Hence the set of $p \geq 1$ such that $\lim _{k \rightarrow+\infty} \operatorname{Mod}_{p}\left(\mathcal{F}^{g}, G_{k}\right)=+\infty$ is open in $[1,+\infty)$.

Proposition 8.5. One has $Q_{m}>1$ if and only if $Z$ has no local cut point.

Proof. The above corollary shows that $Q_{m}>1$ is equivalent to the fact that for $p=1$ one has $\lim _{k \rightarrow+\infty} \operatorname{Mod}_{p}\left(\mathcal{F}^{g}, G_{k}\right)=+\infty$.

Assume $z_{0}$ is a local cut point of $Z$, and let $\eta \subset Z$ be a curve containing $z_{0}$ in its interior. Enlarging $\eta$ if necessary, we may assume that for $\epsilon>0$ small enough, the curve family $U_{\epsilon}(\eta)$ is contained in a family $\mathscr{F}^{g}$ of generic curves. Therefore for any

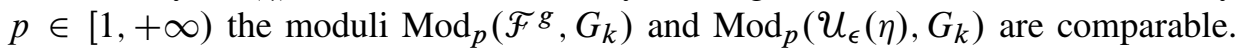
Since $z_{0}$ is a local cut point, every curve which belongs to $U_{\epsilon}(\eta)$ passes through $z_{0}$, provided $\epsilon$ is small enough. Choose for every $k \in \mathbb{N}$ an element $v_{k} \in G_{k}^{0}$ containing $z_{0}$. The function of $G_{k}^{0}$ whose value is 1 on $v_{k}$ and 0 otherwise, is a $U_{\epsilon}(\eta)$-admissible function of $p$-mass 1 . Hence the sequence $\left\{\operatorname{Mod}_{p}\left(U_{\epsilon}(\eta), G_{k}\right)\right\}_{k \in \mathbb{N}}$ is bounded.

Conversely assume that $Z$ has no local cut point. Let $\mathcal{F}^{g}$ be a family of generic curves and let $\eta \subset Z, \epsilon>0$ such that $U_{\epsilon}(\eta) \subset \mathscr{F}^{g}$. Since $Z$ has no local cut point, a construction of J. Mackay [Mac10] shows that the family $U_{\epsilon}(\eta)$ contains an infinite collection of pairwise disjoint curves. It implies obviously that $\lim _{k \rightarrow+\infty} \operatorname{Mod}_{p}\left(U_{\epsilon}(\eta), G_{k}\right)=+\infty$ for $p=1$. 
8.2. A sufficient condition for the CLP. As in the previous subsection $\Gamma$ is a hyperbolic Coxeter group with connected boundary, and $\partial \Gamma$ is equipped with a self-similar metric.

Among the curve families already considered, the combinatorial modulus of generic curves is the lowest one, while the combinatorial modulus of $\mathscr{F}_{0}$ is the largest one. Intermediate curve families are the $\mathscr{F}_{r}(\mathscr{L})$ 's introduced in Section 6.

In the following statement we allow $\mathscr{L}$ to be the empty collection $\emptyset$. In this case we set $\widetilde{F}_{r}(\emptyset):=\mathscr{F}_{0}$ and $\operatorname{Confdim}(\emptyset):=1$. The critical exponents $Q_{M}$ and $Q_{m}$ are respectively defined in Paragraphs 3 and 8.1.

Theorem 8.6. Let $\mathcal{F}^{g}$ be a family of generic curves and let $\mathscr{L}$ be a $\Gamma$-invariant collection of connected proper parabolic limit sets. Assume that for every pair of distinct elements $\partial P, \partial Q \in \mathscr{L}$, the set $\partial P \cap \partial Q$ is totally disconnected or empty. Suppose that there exists $p \in[1,+\infty)$ satisfying

$$
p>\operatorname{Confdim}(\mathscr{L}) \text { and } Q_{m} \leq p \leq Q_{M},
$$

such that for $r>0$ small enough and for every $k \in \mathbb{N}$,

$$
\operatorname{Mod}_{p}\left(\mathscr{F}_{r}(\mathscr{L}), G_{k}\right) \leq D \operatorname{Mod}_{p}\left(\mathcal{F}^{g}, G_{k}\right),
$$

with $D=D(r) \geq 1$ independent of $k$. Then $p=Q_{M}$ and $\partial \Gamma$ satisfies the combinatorial Loewner property.

This leads to

Corollary 8.7. Assume that $\operatorname{Confdim}(\partial \Gamma)>1$, and suppose that for every proper, connected, parabolic limit set $\partial P \subset \partial \Gamma$, one has

$$
\operatorname{Confdim}(\partial P)<\operatorname{Confdim}(\partial \Gamma) \text {. }
$$

Suppose furthermore that for every pair $\partial P, \partial Q$ of distinct, proper, connected, parabolic limit sets, the subset $\partial P \cap \partial Q$ is totally disconnected or empty. Then $\partial \Gamma$ satisfies the CLP.

Proof. One knows that $Q_{M}=\operatorname{Confdim}(\partial \Gamma)$ from Remark 2 at the end of Section 3. Therefore the statement follows from Theorem 8.6 by considering the collection $\mathscr{L}$ of all proper connected parabolic limit sets.

The rest of the paragraph is devoted to the proof of the theorem. We will slightly abuse notation writing $L_{k}$ instead of $C \cdot L_{k}$ where $C$ is the constant appearing in the statement of Proposition 8.3. With this convention the weak submultiplicative inequality in Proposition 8.3 simply writes

$$
L_{k+\ell} \leq L_{k} \cdot \sum_{n=0}^{\ell} L_{\ell-n} b^{n} .
$$

Letting $u_{\ell}:=\sum_{n=0}^{\ell} L_{\ell-n} b^{n}$, it becomes $L_{k+\ell} \leq L_{k} \cdot u_{\ell}$. 
Lemma 8.8. Let $p \in[1,+\infty)$ and $a \in(0,1)$ such that $\sum_{\ell=0}^{+\infty} u_{\ell}^{p} a^{\ell}=+\infty$. Then the sequence $\left\{L_{k}\right\}_{k \in \mathbb{N}}$ tends to $+\infty$ exponentially fast.

Proof. Observe that $\left\{u_{k}\right\}_{k \in \mathbb{N}}$ is submultiplicative. Indeed from its definition and with the weak submultiplicative inequality one has

$$
u_{k+\ell}=\left(\sum_{n=0}^{\ell-1} L_{k+\ell-n} b^{n}\right)+b^{\ell} u_{k} \leq\left(\sum_{n=0}^{\ell-1} L_{\ell-n} b^{n}\right) u_{k}+b^{\ell} u_{k} .
$$

The weak submultiplicative inequality applied with $k=\ell=0$ shows that $L_{0} \geq 1$. With the above inequalities we get that $u_{k+\ell} \leq u_{k} \cdot u_{\ell}$.

Therefore there exists $\alpha \in \mathbb{R}_{+}$such that $\lim _{k \rightarrow+\infty} \frac{\log u_{k}}{k}=\log \alpha$. With our hypotheses we obtain that $\alpha>1$. It follows that there exists a constant $C \geq 1$ such that for every $k \in \mathbb{N}$,

$$
C^{-1} \alpha^{\frac{3}{4} k} \leq u_{k} \leq C \alpha^{\frac{5}{4} k} .
$$

On the other hand one has for every $k, \ell \in \mathbb{N}$ that

$$
u_{k+\ell}=\left(\sum_{n=0}^{\ell-1} L_{k+\ell-n} b^{n}\right)+b^{\ell} u_{k} \leq L_{k}\left(\sum_{n=0}^{\ell-1} u_{\ell-n} b^{n}\right)+b^{\ell} u_{k} .
$$

Because $b \in(0,1)$, the above inequalities and a simple computation show the existence of constants $C_{1}, C_{2} \geq 1$ with

$$
\alpha^{\frac{3}{4}(k+\ell)} \leq C_{1} L_{k} \alpha^{\frac{5}{4} \ell}+C_{2} \alpha^{\frac{5}{4} k} .
$$

Letting $k=\ell$ yields the desired conclusion.

Proof of Theorem 8.6. Let $p$ satisfy the hypotheses of the statement. At first we prove that the sequence $\left\{L_{k}\right\}_{k \in \mathbb{N}}$ is bounded. Since $p \leq Q_{M}$ part (ii) of Corollary 3.7 shows that the sequence $\left\{\operatorname{Mod}_{p}\left(\widetilde{F}_{0}, G_{k}\right)\right\}_{k \in \mathbb{N}}$ admits a positive lower bound. With our assumptions and with Corollary 6.4 we obtain the existence of constants $C>0$ and $a \in(0,1)$ such that for every $k \in \mathbb{N}$,

$$
C \leq \sum_{\ell=0}^{k} m_{k-\ell} a^{\ell}=\sum_{\ell=0}^{k} L_{k-\ell}^{-p} a^{\ell}
$$

In particular $\left\{L_{k}\right\}_{k \in \mathbb{N}}$ does not tend to $+\infty$, and so according to the previous lemma the sum $\sum_{\ell=0}^{+\infty} u_{\ell}^{p} a^{\ell}$ is finite. The weak submultiplicativity inequality writes $L_{k-\ell} \geq$ $L_{k} \cdot u_{\ell}^{-1}$, and hence

$$
C \leq L_{k}^{-p} \cdot \sum_{\ell=0}^{k} u_{\ell}^{p} a^{\ell}
$$

Therefore $\left\{L_{k}\right\}_{k \in \mathbb{N}}$ is bounded. 
We now claim that $\left\{L_{k}\right\}_{k \in \mathbb{N}}$ admits a positive lower bound. For this purpose observe that from its definition and with the weak submultiplicative inequality one has

$$
u_{k+\ell}=\left(\sum_{n=0}^{\ell-1} L_{k+\ell-n} b^{n}\right)+b^{\ell} u_{k} \leq L_{k}\left(\sum_{n=0}^{\ell-1} u_{\ell-n} b^{n}\right)+b^{\ell} u_{k} .
$$

Since $\left\{L_{k}\right\}_{k \in \mathbb{N}}$ is bounded so is $\left\{u_{k}\right\}_{k \in \mathbb{N}}$. Let $M$ be an upper bound for $\left\{u_{k}\right\}_{k \in \mathbb{N}}$, we obtain

$$
u_{k+\ell} \leq L_{k} S_{\ell}+b^{\ell} M \quad \text { with } S_{\ell}:=\sum_{n=0}^{\ell-1} u_{\ell-n} b^{n} .
$$

Assume by contradiction that $\liminf _{k \rightarrow+\infty} L_{k}=0$. At first choose $\ell$ such that $b^{\ell} M<1 / 2$, then pick $k$ such that $L_{k} S_{\ell}<1 / 2$; we get that $u_{k+\ell}<1$. As seen already in the proof of Corollary 8.4, this implies that $\left\{L_{k}\right\}_{k \in \mathbb{N}}$ tends to 0 . Since $p \geq Q_{m}$ it contradicts Corollary 8.4 and the claim follows.

The claim combined with our assumption imply that $\operatorname{Mod}_{p}\left(\mathcal{F}_{r}(\mathscr{L}), G_{k}\right)$ is bounded independently of $k$. With Corollary 6.4 we get that the same holds for $\operatorname{Mod}_{p}\left(\mathcal{F}_{0}, G_{k}\right)$. Summarizing there exits a constant $C>0$ such that for every $k \in \mathbb{N}$,

$$
C^{-1} \leq \operatorname{Mod}_{p}\left(\mathcal{F}^{g}, G_{k}\right) \leq \operatorname{Mod}_{p}\left(\mathscr{F}_{0}, G_{k}\right) \leq C
$$

The $p$-combinatorial Loewner property comes now from Lemma 8.1 and from Propositions 2.9 and 3.4. Note that Proposition 3.4 requires that $p>1$. This inequality is satisfied because by assumption $p>\operatorname{Confdim}(\mathscr{L}) \geq 1$. Finally one has $p=Q_{M}$ according to Corollary 3.7 .

Remarks and questions. 1) The equality $Q_{M}=Q_{m}$ is a necessary condition for the CLP. This follows from Remark 1 at the end of Section 3, by choosing the continua $A, B$ in order that $\mathcal{F}(A, B)$ is contained in a family of generic curves.

2) Recall that $Q_{M}$ is a quasi-Möbius invariant of $Z$ (see Remark 2 at the end of Section 3). Is it true for $Q_{m}$ too?

\section{Examples}

We give various examples of boundaries of hyperbolic Coxeter groups satisfying the combinatorial Loewner property.

9.1. Simplex groups. Let $(\Gamma, S)$ be an infinite hyperbolic Coxeter group such that every proper parabolic subgroup is finite. This last condition is equivalent to the fact that the Davis chamber of $(\Gamma, S)$ is a simplex of dimension $|S|-1$ (see [Dav08]). Using Proposition 8.5 one sees easily that $Q_{m}>1$ if and only if $|S| \geq 4$. Since 
the only infinite parabolic subgroup is $\Gamma$ itself, the assumptions of Corollary 8.7 are clearly satisfied for $|S| \geq 4$. Therefore when $|S| \geq 4$, the boundary of $\Gamma$ admits the CLP.

This result was already known by other methods. Indeed it is a well-known theorem due to Lannér that such a group acts by isometries, properly discontinuously and cocompactly on the real hyperbolic space $\mathbb{H}^{n}$ of dimension $n=|S|-1$. Moreover the boundary of $\mathbb{H}^{n}$ is the Euclidean $(n-1)$-sphere which is a Loewner space for $n-1 \geq 2$ (see [HK98]).

9.2. Prism groups. Let $n \geq 3$, consider an $n$-simplex and truncate an open neighborhood of one of its vertices. The resulting polytope is an $n$-prism (i.e. isomorphic to the product of a segment with an $(n-1)$-simplex). Let $\Sigma \subset \mathbb{H}^{n}$ be a geodesic $n$-prism whose dihedral angles are submultiples of $\pi$, and those of the truncated face are equal to $\frac{\pi}{2}$. Such polytopes have been classified by Kaplinskii ([Kap74], see also [Vin85], table 4), they exist only when $n \leq 5$. Let $\Gamma$ be the discrete subgroup of $\operatorname{Isom}\left(\mathbb{H}^{n}\right)$ generated by reflections along the codimension 1 faces of $\Sigma$ except the truncated one. The subset $\Gamma \cdot \Sigma$ is equal to $\mathbb{H}^{n}$ minus a countable union of disjoint totally geodesic half-spaces. Therefore $\Gamma$ is a (word) hyperbolic Coxeter group and its boundary can be identified with $S^{n-1}$ minus a countable union of disjoint Euclidean $(n-1)$-balls.

Up to conjugacy the only infinite proper parabolic subgroup is the simplex group generated by the faces adjacent to the truncated one. Its limit set is an Euclidean $(n-2)$-sphere. Let $\mathscr{L}$ be the collection of all proper parabolic limit sets. It follows that $\operatorname{Confdim}(\mathscr{L})=n-2$ and that $\partial P \cap \partial Q=\emptyset$ for every distinct elements $\partial P, \partial Q \in \mathscr{L}$. Therefore the following lemma, in combination with Corollary 8.7 and the fact that Confdim $(\partial \Gamma)=Q_{M}$ (see Remark 2 at the end of Section 3), shows that $\partial \Gamma$ admits the CLP.

Lemma 9.1. One has $Q_{M}>n-2$.

Proof. When $n=3$ this follows from Proposition 8.5 since in this case $\partial \Gamma$ is homeomorphic to the Sierpinski carpet which doesn't possess any local cut point.

In general we will prove that $\partial \Gamma$ equipped with the induced Euclidean metric satisfies $Q_{m}>n-2$. Since $Q_{m} \leq Q_{M}$ and since $Q_{M}$ is a quasi-Möbius invariant (see Remark 2 at the end of Section 3), the lemma will follow. For this purpose we will exhibit a collection $\left\{F_{i}\right\}_{i \in \mathbb{N}}$ of pairwise disjoint closed subsets of $\partial \Gamma$ with the following properties:

(1) The $F_{i}$ 's are uniformly bi-Lipschitz equivalent to the unit Euclidean sphere $S^{n-2}$, i.e. for every $i \in \mathbb{N}$ there is a $C$-bi-Lipschitz homeomorphism from $F_{i}$ to $S^{n-2}$ where $C>0$ is independent of $i$.

(2) There exists a constant $D$ with $d_{0} \leq D \leq \frac{1}{2} \operatorname{diam}\left(F_{i}\right)$ such that for every $i \in \mathbb{N}$ one has $\mathcal{F}_{i}:=\left\{\gamma \subset F_{i} ; \operatorname{diam}(\gamma) \geq D\right\} \subset \mathcal{F}^{g}$. 
As we shall see, existence of such a collection implies that

$$
\lim _{k \rightarrow+\infty} \operatorname{Mod}_{n-2}\left(\mathcal{F}^{g}, G_{k}\right)=+\infty
$$

which in turn implies that $Q_{m}>n-2$ thanks to Corollary 8.4.

To establish the relation (9.1), notice first that $S^{n-2}$ admits the CLP with exponent $n-2$ (see Theorem 2.6.1). Therefore item (1) implies that $\operatorname{Mod}_{n-2}\left(\mathcal{F}_{i}, G_{k}\right)$ is bounded away from 0 independently of $(i, k) \in \mathbb{N}^{2}$. For every $k \in \mathbb{N}$ pick a maximal subcollection $\left\{F_{k_{i}}\right\}_{i=1}^{n_{k}} \subset\left\{F_{i}\right\}_{i \in \mathbb{N}}$ such that every $v \in G_{k}^{0}$ intersects at most one of the $F_{k_{i}}$ 's. Then we get with item (2) that

$$
\operatorname{Mod}_{n-2}\left(\mathcal{F}^{g}, G_{k}\right) \gtrsim \sum_{i=1}^{n_{k}} \operatorname{Mod}_{n-2}\left(\widetilde{F}_{k_{i}}, G_{k}\right) \gtrsim n_{k} .
$$

Since $n_{k}$ tends to $+\infty$ the relation (9.1) follows.

We now construct the collection $\left\{F_{i}\right\}_{i \in \mathbb{N}}$. Pick a wall $M$ of $\Gamma$, its limit set in $\partial \mathbb{H}^{n}$ is contained in an $(n-2)$-sphere. Identifying $\partial \mathbb{H}^{n}$ with the unit $(n-1)$-Euclidean sphere, we may assume that $\partial M$ is contained in the equator. Let $\mathcal{B}$ be the set of connected components of $\partial \mathbb{H}^{n} \backslash \partial \Gamma$. Because $\partial \Gamma$ is invariant by the reflection along $M$, every ball $B \in \mathcal{B}$ with $\bar{B} \cap \partial M \neq \varnothing$ is centered on the equator. Thus by adding to $\partial M$ a countable union of disjoint $(n-2)$-half-spheres contained in the north hemisphere, we obtain a subset $F \subset \partial \Gamma$ homeomorphic to $S^{n-2}$. Since the parabolic subgroups are quasi-convex in $\Gamma$, there exists a constant $C>0$ such that for any distinct $B, B^{\prime} \in \mathscr{B}$ one has $\Delta\left(B, B^{\prime}\right) \geq C$ (see [Bou04], p. 89). One deduces easily from this fact that $F$ is bi-Lipschitz-equivalent to $S^{n-2}$.

We construct $F_{i}$ by induction, as a small deformation of $F$. Let $S_{0}$ be a smooth manifold diffeomorphic to $S^{n-2}$, contained in the north hemisphere, disjoint from $F$ and lying within small Hausdorff distance from $F$. For every $B \in \mathscr{B}$ with $B \cap S_{0} \neq \emptyset$, replace $B \cap S_{0}$ by the spherical boundary of the connected component of $B \backslash S_{0}$ which does not contain the center of $B$. Let $F_{0}$ be the resulting subset of $\partial \Gamma$. Since the balls $B \in \mathscr{B}$ are pairwise disjoint, $F_{0}$ is still a topological $(n-2)$-sphere disjoint from $F$. Only finitely many $B \in \mathscr{B}$ have diameter larger than a given positive constant. Therefore by taking $S_{0}$ close enough to $F$, one can ensure that $F_{0}$ lies within arbitrary small Hausdorff distance from $F$. Note that for $B \in \mathcal{B}$ the frontier of $B \cap S_{0}$ in $S_{0}$ belongs to $F_{0}$. Their union (for $B \in \mathscr{B}$ ) is called the singular locus of $F_{0}$. We claim that one can choose $S_{0}$ properly in order that $F_{0}$ is bi-Lipschitz equivalent to $S^{n-2}$ with controlled Lipschitz constants. Indeed the bi-Lipschitz regularity holds provided the dihedral angles of the singular locus in $F_{0}$ admit an uniform positive lower bound. Using the previous lower bound estimates for the relative distances between elements of $\mathcal{B}$, one can construct $S_{0}$ in such a way that at every location its curvatures are smaller than those of the balls $B \in \mathscr{B}$ with $B \cap S_{0} \neq \varnothing$. The control on the dihedral angles follows, which in turn implies the claim. We repeat this procedure starting with a manifold $S_{1}$ whose Hausdorff distance to $F$ is much 
smaller than the one between $F_{0}$ and $F$, in order that the resulting subset $F_{1} \subset \partial \Gamma$ is disjoint from $F_{0}$. The construction of the $F_{i}$ 's is now clear.

9.3. Highly symmetric Coxeter groups. Let $L$ be a finite graph whose girth is greater or equal to 4 and such that the valency of each vertex is at least equal to 3 . Let $r$ be an even integer greater or equal to 6 . Consider the Coxeter system $(\Gamma, S)$ with one generating reflection for every vertex in $L$, and such that the order of the product of two distinct vertices $v, w$ is $r / 2$ if $(v, w) \in L^{1}$, and $+\infty$ otherwise. Its Davis complex is a 2-cell contractible complex $X$ where every 2-cell is isomorphic to the regular right-angled $r$-gon in $\mathbb{H}^{2}$, and where the link of every vertex is isomorphic to $L$. Equipped with the geodesic distance induced by its 2-cells, $X$ is a CAT(-1)space on which $\Gamma$ acts properly discontinuously, by isometries, and cocompactly. In particular $\Gamma$ is a hyperbolic group. Moreover the Cayley graph of $(\Gamma, S)$ can be identified with the 1-skeleton of $X$. The walls of $(\Gamma, S)$ identify with totally geodesic subtrees of $X$, namely those generated by a geodesic segment which joins the middles of two opposite edges in a 2-cell. We shall call them the walls of $X$. Consider $X$ minus the union of its walls, the closures of the connected components are called the Davis chambers of $X$. Each of them is homeomorphic to the cone over $L$ (see [Dav08], [Hag98] for more details).

Assume now that $L$ is a highly symmetric graph in the following sense: for every pair of adjacent vertices $v, w \in L^{0}$ the pointwise stabilizer of the star of $v$ acts transitively on the set of remaining edges in the star of $w$. Recall that the star of a vertex is the union of its incident edges. Examples of such graphs include the full bipartite graph with $s+t$ vertices $(s, t \geq 3)$, the Moufang generalized polygons (see [Ron89]), every 3-transitive trivalent graph (such as the Petersen graph), the odd graphs (see [Big74]), etc.

We have

Proposition 9.2. $\partial \Gamma$ satisfies the $C L P$.

When $L$ is a generalized polygon the statement was already known. Indeed in this case $\partial \Gamma$ possesses a self-similar metric $\delta$ such that $(\partial \Gamma, \delta)$ is an Loewner space [BP00].

Proof. Let $K \leqq \operatorname{Isom}(X)$ be the pointwise stabilizer of the Davis chamber containing the identity of $\Gamma$. Endow $\partial \Gamma$ with the self-similar metric $d$ induced by a visual metric on $\partial X$; then $K$ acts on $(\partial \Gamma, d)$ by bi-Lipschitz homeomorphisms.

We will show that the assumptions of Theorem 8.6 are satisfied with $\mathscr{L}=\emptyset$. Since $\partial \Gamma$ does not admit any local cut point - it is homeomorphic to the Menger curve, see [KaK00] - Proposition 8.5 implies that $Q_{M}>1=\operatorname{Confdim}(\mathscr{L})$. Let $p>1$ with $Q_{m} \leq p \leq Q_{M}$.

Let $I \subset S$ be such that $\partial \Gamma_{I}$ is a connected parabolic limit set. We will prove by induction on $|S|-|I|$, that for $\delta, r>0$ small enough, there exists a constant $C \geq 1$ 
such that for every $k \in \mathbb{N}$ one has

$$
\operatorname{Mod}_{p}\left(\mathcal{F}_{\delta, r}\left(\partial \Gamma_{I}\right), G_{k}\right) \leq C \operatorname{Mod}_{p}\left(\mathcal{F}^{g}, G_{k}\right) .
$$

For $|S|-|I|=0$ there is nothing to prove. Assume that inequality (9.2) holds for $|S|-|I| \leq N$. We wish to prove it for $|S|-|I| \leq N+1$. Pick a curve $\gamma \subset \partial \Gamma_{I}$ which crosses every $\partial M_{v}$ for $v \in I$, and such that for $\delta, r, \epsilon$ small enough $\mathscr{F}_{\delta, r}\left(\partial \Gamma_{I}\right)$ and $U_{\epsilon}(\gamma)$ have comparable modulus (see Corollary 6.2). Using the group $K$ we will deform $\gamma$ into a curve contained in a larger parabolic limit set. To do so we first need some information on the dynamics of the action of $K$ on $\partial \Gamma$.

Let $v \in I$ and consider a special subgroup $\Gamma_{J}$ with $v \in J$. Its Davis complex, denoted by $X_{J}$, embeds equivariantly and totally geodesically in $X$. The wall of $v$ in $X_{J}$ is equal to $M_{v} \cap X_{J}$, where $M_{v}$ denotes the wall of $v$ in $X$. Hence we see that either $\partial \Gamma_{J} \cap \partial M_{v}$ is of empty interior in $\partial M_{v}$, or $J$ contains all the neighbors of $v$ in $L$. For $v \in S$, let $U_{v}$ be the open dense subset of $\partial M_{v}$ whose complement is the union of all empty interior subsets of the form $\partial \Gamma_{J} \cap \partial M_{v}$. One has:

Lemma 9.3. Every orbit of the $K$-action on $\Pi_{v \in S} \partial M_{v}$ is open.

Assuming the lemma we finish the proof of the proposition. Thanks to the lemma there exists $g \in K$ such that for every $v \in I$ one has $g \gamma \cap U_{v} \neq \emptyset$. Let $Q \leqq \Gamma$ be a parabolic subgroup such that $g \gamma \subset \partial Q$. Enlarging $\gamma$ if necessary we may assume that its convex hull contains the identity of $\Gamma$. This property remains for $g \gamma$, thus $Q$ is a special subgroup, $\Gamma_{J}$ say. Since $g \gamma$ crosses every $\partial M_{v}$ for $v \in I$, one has $I \subset J$ (see Remark 1 at the end of Section 5). Therefore the above discussion implies that $J$ contains the neighbors in $L$ of every vertex $v \in I$. Hence, assuming $|I|<|S|$, one obtains that $|I|<|J|$. Moreover, since $g$ is bi-Lipschitz, $U_{\epsilon}(\gamma)$ and $U_{\epsilon}(g \gamma)$ have comparable modulus. Therefore Corollary 6.2 combined with the induction assumption give the expected inequality (9.2).

It remains to give the

Proof of Lemma 9.3. Let $\Sigma \subset X$ be the Davis chamber containing the identity of $\Gamma$. For a pair of adjacent vertices $v, v^{\prime} \in L^{0}$ consider the walls $M, M^{\prime} \subset X$ of the reflections $v, v^{\prime}$. They intersect exactly at the center $o \in X$ of the 2-cell corresponding to the edge $\left(v, v^{\prime}\right) \in L^{1}$. Denote by $H_{-}, H_{+}$the closed half-spaces bounded by $M$ with $\Sigma \subset H_{-}$. We will show that the pointwise stabilizer of $H_{+}$in $\operatorname{Isom}(X)$ acts transitively on the set of the edges of $M^{\prime} \cap H_{-}$adjacent to the one containing $o$. Since $v, v^{\prime}$ is an arbitrary pair of adjacent vertices, this result combined with the $\Gamma$-action on $X$ gives the lemma. To do so we shall use an argument of F. Haglund ([Hag98], proof of Proposition A.3.1). For simplicity assume first that $r$ is a multiple of 4. Every automorphism of $L$ gives rise to a group automorphism of $\Gamma$ which in turn induces an isometry of $X$. Let $a \in \operatorname{Aut}(L)$ which pointwise stabilizes the star of $v$. Then the associated isometry $f$ acts trivially on the wall $M$ (the hypothesis that $r$ is a multiple 
of 4 is used at this point). Therefore one can define $g \in \operatorname{Isom}(X)$ by letting $g=\mathrm{id}$ on $H_{+}$and $g=f$ on $H_{-}$. Since $L$ is highly symmetric the result follows.

When $r$ is only a multiple of $2, f$ is not anymore the identity on $M$ but still it acts trivially on a tree $T$ containing $o$ and which zig-zag in the 2-cells (joining the center to the middles of two edges at even distance). Moreover one can choose $T$ such that $H_{+}$and $M^{\prime} \cap H_{-}$are contained in the closure of two distinct connected components of $X \backslash T$. Hence the previous argument generalizes.

9.4. More planar examples. Let $\Gamma$ be a hyperbolic Coxeter group with planar connected boundary. Planarity is exploited to define transversal intersection of parabolic limit sets. Some modulus estimates are derived in Proposition 9.5. They are useful to establish the CLP in some examples.

Definition 9.4. Two curves $\gamma_{1}, \gamma_{2} \subset \partial \Gamma$ intersect transversely if there exists $\epsilon>0$ such that every pair of curves $\eta_{1} \in \mathcal{U}_{\epsilon}\left(\gamma_{1}\right), \eta_{2} \in U_{\epsilon}\left(\gamma_{2}\right)$ intersect. We say that two connected parabolic limit sets $\partial P_{1}, \partial P_{2} \subset \partial \Gamma$ intersects transversely if there exists two curves $\gamma_{1} \subset \partial P_{1}, \gamma_{2} \subset \partial P_{2}$ which intersect transversely.

Proposition 9.5. Let $\partial P_{1}, \partial P_{2}$ be two connected parabolic limit sets which intersect transversely and let $\partial Q$ be the smallest parabolic limit set containing $\partial P_{1} \cup \partial P_{2}$. Then for every $p \geq 1$ and for $\delta, r$ small enough one has

$$
\inf _{i=1,2} \operatorname{Mod}_{p}\left(\mathcal{F}_{\delta, r}\left(\partial P_{i}\right), G_{k}\right) \asymp \operatorname{Mod}_{p}\left(\mathcal{F}_{\delta, r}(\partial Q), G_{k}\right) .
$$

Proof. Pick a pair of curves $\gamma_{1} \subset \partial P_{1}, \gamma_{2} \subset \partial P_{2}$ as in the definition. Extending them if necessary, we may assume that $\partial P_{i}$ is the smallest parabolic limit set containing $\gamma_{i}$. By Theorem 6.1 and Corollary 6.2 one has for $\epsilon, \delta, r>0$ small enough:

$$
\operatorname{Mod}_{p}\left(U_{\epsilon}\left(\gamma_{i}\right), G_{k}\right) \asymp \operatorname{Mod}_{p}\left(\mathcal{F}_{\delta, r}\left(\partial P_{i}\right), G_{k}\right) \gtrsim \operatorname{Mod}_{p}\left(\mathcal{F}_{\delta, r}(\partial Q), G_{k}\right) .
$$

Assume by contradiction that for $i=1,2$ the $\operatorname{modulus}^{\operatorname{Mod}_{p}}\left(U_{\epsilon}\left(\gamma_{i}\right), G_{k}\right)$ is large compared to $\operatorname{Mod}_{p}\left(\mathcal{F}_{\delta, r}(\partial Q), G_{k}\right)$. Let $\rho: G_{k} \rightarrow \mathbb{R}_{+}$be a $\mathcal{F}_{\delta, r}(\partial Q)$-admissible function with minimal $p$-mass. Then for $i=1,2$ the family $U_{\epsilon}\left(\gamma_{i}\right)$ contains a curve $\eta_{i}$ of small $\rho$-length (see Lemma 2.7). By transversality the curves $\eta_{1}$ and $\eta_{2}$ intersect, thus $\widetilde{F}_{\delta, r}(\partial Q)$ contains a curve of small $\rho$-length, contradicting the admissibility of $\rho$. Therefore the moduli $\inf _{i=1,2} \operatorname{Mod}_{p}\left(U_{\epsilon}\left(\gamma_{i}\right), G_{k}\right)$ and $\operatorname{Mod}_{p}\left(\mathcal{F}_{\delta, r}(\partial Q), G_{k}\right)$ are comparable, so the proposition follows from Corollary 6.2.

As an illustration consider a 3-dimensional cube and truncate an open neighborhood of every vertex. The resulting polyhedron possesses eight triangular faces and six hexagonal ones. Let $\Sigma \subset \mathbb{H}^{3}$ be a regular geodesic truncated cube whose dihedral angles are submultiples of $\pi$, and those of the triangular faces are equal to $\frac{\pi}{2}$. By regular we mean that $\Sigma$ admits all the cube symmetries. Let $\Gamma$ be the discrete subgroup of $\operatorname{Isom}\left(\mathbb{H}^{3}\right)$ generated by reflections along the hexagonal faces of $\Sigma$. The subset 
$\Gamma \cdot \Sigma$ is equal to $\mathbb{H}^{3}$ minus a countable disjoint union of totally geodesic half-spaces. Therefore $\Gamma$ is a (word) hyperbolic Coxeter group whose boundary is homeomorphic to the Sierpinski carpet. We will check that $\partial \Gamma$ satisfies the CLP.

For this purpose, equip $\partial \Gamma$ with a self-similar metric $d$ such that the symmetries of the cube act on $(\partial \Gamma, d)$ by bi-Lipschitz homeomorphisms.

Let $L$ be the graph whose vertices are the generators of $\Gamma$ and whose edges are the pairs $\left(s, s^{\prime}\right)$ with $s \neq s^{\prime}$ and $s s^{\prime}$ of finite order. Then $L$ is the 1-skeleton of the octahedron. One sees easily that there is only one type of proper parabolic subgroups with connected non-circular limit set: those whose graph is equal to $L$ minus a vertex and its adjacent edges. By applying an appropriate cube symmetry to such a parabolic $P$, we get another parabolic $P^{\prime}$ such that $\partial P$ and $\partial P^{\prime}$ intersect transversely. Indeed the graph of $P$ contains a circuit of length 4 , and any two distinct circuits of length 4 in $L$ give rise to circular parabolic limit sets that intersect transversely. Moreover the smallest parabolic limit set containing $\partial P \cup \partial P^{\prime}$ is $\partial \Gamma$ itself. Hence Proposition 9.5 and the Lipschitz invariance of $d$, show that $\mathcal{F}_{\delta, r}(\partial P)$ and $\mathcal{F}^{g}$ have comparable modulus. The CLP comes now from Theorem 8.6 with $\mathscr{L}$ equal to the collection of all circular parabolic limit sets.

Remarks and questions. 1) In [Ben06] Y. Benoist exhibits examples of hyperbolic Coxeter groups with the following properties:

- Their Davis chambers are isomorphic to the product of two simplices of dimension 2.

- They are virtually the fundamental group of a compact locally CAT(-1) 4-dimensional manifold.

- They are not quasi-isometric to $\mathbb{H}^{4}$.

- They admit a properly discontinuous cocompact projective action on a strictly convex open subset of the real projective space $\mathbb{P}^{4}$.

An interesting question is to determine whether their boundary satisfies the CLP or even the analytical Loewner property. Previous examples of hyperbolic Coxeter groups enjoying the first three properties above appear in Moussong's thesis (unpublished, see [Dav08], Example 12.6.8). In connection with these problems, we remark that F. Esselmann has classified the Coxeter polytopes in $\mathbb{H}^{4}$ isomorphic to the product of two simplices of dimension 2 (see [Ess96]).

2) Suppose $Z$ is a Sierpinski carpet sitting in the standard 2-sphere. M. Bonk ([Bon06], Proposition 7.6) has shown that if the peripheral circles of $Z$ are uniformly quasi-Möbius homeomorphic to the standard circle, and have pairwise relative distance bounded away from zero, then $Z$ satisfies a version of the Loewner property for transboundary 2-modulus. Since a Sierpinski carpet Coxeter group boundary $\partial \Gamma$ is quasi-Möbius homeomorphic to such a $Z$, this should imply that $\partial \Gamma$ satisfies a transboundary variant of the CLP for 2-modulus. It would be interesting to have examples which do not satisfy the (usual) CLP. 


\section{0. $\ell_{p}$-equivalence relations}

This section covers some applications of the previous results to $\ell_{p}$-equivalence relations. These equivalence relations are of great interest because of their invariance by quasi-Möbius homeomorphisms. Moreover they can be used to provide examples of spaces which do not admit the CLP (see the remark at the end of the section).

We start by defining the $\ell_{p}$-equivalence relation, it requires some preliminary materials. Let $Z$ be a compact doubling metric space. Assume in addition that it is uniformly perfect, i.e. there exists a constant $0<\lambda<1$ such that for every ball $B(z, r)$ of $Z$ with $0<r \leq \operatorname{diam} Z$ one has $B(z, r) \backslash B(z, \lambda r) \neq \emptyset$.

We will associate to $Z$ a Gromov hyperbolic graph $G$ such that $\partial G$ - namely its boundary at infinity - can be identified canonically with $Z$. For this purpose fix a constant $\kappa \geq 1$, and pick for each $k \in \mathbb{N}^{*}$ a finite covering $U_{k}$ of $Z$ with the following properties:

- For every $v \in U_{k}$ there exists $z_{v} \in Z$ such that

$$
B\left(z_{v}, \kappa^{-1} 2^{-k}\right) \subset v \subset B\left(z_{v}, \kappa 2^{-k}\right) .
$$

- For all distinct $v, w \in U_{k}$ one has

$$
B\left(z_{v}, \kappa^{-1} 2^{-k}\right) \cap B\left(z_{w}, \kappa^{-1} 2^{-k}\right)=\emptyset .
$$

- For every $z \in Z$ there exists $v \in U_{k}$ with $B\left(z, \kappa^{-1} 2^{-k}\right) \subset v$.

Let $U_{0}=\{Z\}$ be the trivial cover, and let $G$ be the graph whose vertex set is $\bigcup_{k \in \mathbb{N}} U_{k}$ and whose edges are defined as follows: two distinct vertices $v$ and $w$ are joined by an edge if

- $v$ and $w$ both belong to $\mathcal{U}_{k},(k \in \mathbb{N})$, and $v \cap w \neq \emptyset$, or if

- one belongs to $\mathcal{U}_{k}$, the other one belongs to $\mathcal{U}_{k+1}$, and $v \cap w \neq \emptyset$.

Then $G$ is a Gromov hyperbolic graph with bounded valency. The metric space $Z$ is bi-Lipschitz equivalent to $\partial G$ equipped with a visual metric [BP03].

For a countable set $E$ and for $p \in[1, \infty)$, we denote by $\ell_{p}(E)$ the Banach space of $p$-summable real functions on $E$. The first $\ell_{p}$-cohomology group of $G$ is

$$
\ell_{p} H^{1}(G)=\left\{f: G^{0} \rightarrow \mathbb{R} ; d f \in \ell_{p}\left(G^{1}\right)\right\} / \ell_{p}\left(G^{0}\right)+\mathbb{R},
$$

where $d f$ is the function on $G^{1}$ defined by

$$
d f(a)=f\left(a_{+}\right)-f\left(a_{-}\right) \quad \text { for all } a \in G^{1},
$$

and where $\mathbb{R}$ denotes the set of constant functions on $G^{0}$. Equipped with the seminorm induced by the $\ell_{p}$-norm of $d f$ the topological vector space $\ell_{p} H^{1}(G)$ is a Banach space. It is a quasi-isometric invariant of $G$ and a quasi-Möbius invariant of $Z$. In addition $\ell_{p} H^{1}(G)$ injects in $\ell_{q} H^{1}(G)$ for $1 \leq p \leq q<+\infty$. See [Gro93], [BP03] for a proof of these results. 
Recall that $Z$ being a compact, doubling, uniformly perfect metric space, it admits a doubling measure, that is a finite measure $\mu$ such that for every ball $B \subset Z$ of positive radius one has $0<\mu(2 B) \leq C \mu(B)$, with $C \geq 0$ independent of $B$ (see [Hei01]). By a result of R. Strichartz [Str83] (see also [Pan89a], [BP03]) there is a continuous monomorphism

$$
\begin{aligned}
\ell_{p} H^{1}(G) & \longleftrightarrow L^{p}(Z, \mu) / \mathbb{R}, \\
{[f] } & \longmapsto f_{\infty} \bmod \mathbb{R},
\end{aligned}
$$

where $f_{\infty}$ is defined $\mu$-almost everywhere as follows: for $z \in Z$ and for any geodesic ray $r_{z}$ of $G$ with endpoint $z$,

$$
f_{\infty}(z)=\lim _{t \rightarrow+\infty} f\left(r_{z}(t)\right)
$$

Following M. Gromov ([Gro93], p. 259, see also [Ele97] and [Bou04]) we set

$$
B_{p}^{0}(Z):=\left\{u: Z \rightarrow \mathbb{R} \text { continuous } ; u=f_{\infty} \text { with }[f] \in \ell_{p} H^{1}(G)\right\},
$$

and we define the $\ell_{p}$-equivalence relation on $Z$ by

$$
x \sim_{p} y \Longleftrightarrow u(x)=u(y) \text { for all } u \in B_{p}^{0}(Z) .
$$

This is a closed equivalence relation which is invariant by the group of quasi-Möbius homeomorphisms of $Z$.

Proposition 10.1. The cosets of the $\ell_{p}$-equivalence relation are continua.

Proof. We will use the following obvious properties of the space $B_{p}^{0}(Z)$.

(i) If $u_{1}$ and $u_{2}$ belong to $B_{p}^{0}(Z)$ then $\max \left\{u_{1}, u_{2}\right\}$ does too.

(ii) Let $u_{1}, u_{2} \in B_{p}^{0}(Z)$, and let $U_{1}, U_{2}$ be open subsets of $Z$ with $U_{1} \cup U_{2}=Z$. Assume that $u_{1}=u_{2}$ on $U_{1} \cap U_{2}$. Then the function $u$ defined by $u=u_{1}$ on $U_{1}$ and $u=u_{2}$ on $U_{2}$, belongs to $B_{p}^{0}(Z)$.

First we claim that for any coset $F$ and any compact subset $K \subset Z$ disjoint from $F$, there exists $u \in B_{p}^{0}(Z)$ such that $u(F)=0$ and $u(K)=1$. Indeed for any $z \in K$ there exists a function $u_{z} \in B_{p}^{0}(Z)$ with $u_{z}(F)=0$ and $u_{z}(z)>1$. Extract a finite cover of $K$ from the open subsets $\left\{u_{z}>1\right\}$. Let $U_{1}, \ldots, U_{n}$ be such a cover and let $u_{1}, \ldots, u_{n}$ be the corresponding functions. Then the function

$$
v=\sum_{i=1}^{n} \max \left\{0, u_{i}\right\}
$$

belongs to $B_{p}^{0}(Z)$ and satisfies $v(F)=0$ and $v \geq 1$ on $K$. Letting $u=\min \{1, v\}$ the claim follows. 
Assume now by contradiction that a coset $F$ is a disjoint union of two non-empty compact subsets $F_{1}$ and $F_{2}$. Let $r>0$. By the previous claim there exists a function $u \in B_{p}^{0}(Z)$ such that $u(F)=0$ and $u\left(Z \backslash N_{r}(F)\right)=1$. For $r$ small enough $N_{r}(F)$ is the disjoint union of $N_{r}\left(F_{1}\right)$ and $N_{r}\left(F_{2}\right)$. Define a function $v$ on $Z$ by letting $v(z)=1$ for $z \in N_{r}\left(F_{2}\right)$, and $v(z)=u(z)$ otherwise. Then the above property (ii) applied to the open subsets $Z \backslash \bar{N}_{r}\left(F_{i}\right)$ shows that the function $v$ belongs to $B_{p}^{0}(Z)$. Moreover it satisfies $v\left(F_{1}\right)=0$ and $v\left(F_{2}\right)=1$. Contradiction.

The following result relates the $\ell_{p}$-equivalence relation with the combinatorial $p$-modulus.

Proposition 10.2. Let $\left\{G_{k}\right\}_{k \in \mathbb{N}}$ be a $\kappa$-approximation of $Z$. If $x, y \in Z$ satisfy $x \varkappa_{p} y$, then there exist open subsets $U, V \subset Z$ containing respectively $x$ and $y$ such that

$$
\lim _{k \rightarrow+\infty} \operatorname{Mod}_{p}\left(U, V, G_{k}\right)=0
$$

Proof. Recall that $Z$ being a doubling metric space, up to a multiplicative constant the $G_{k}$-combinatorial $p$-modulus does not depend on the $\kappa$-approximation (see Proposition 2.2). Consider the graph $G$ associated to a family of covers $\left\{\mathcal{U}_{k}\right\}_{k \in \mathbb{N}}$ as described at the beginning of the section. For $k \in \mathbb{N}$, let $G_{k}$ be the subgraph of $G$ which is the incidence graph of the covering $U_{k}$. The family $\left\{G_{k}\right\}_{k \in \mathbb{N}}$ is a $\kappa$-approximation of $Z$. In addition $G_{k}^{0}$ can be identified with the sphere in $G$ of radius $k$ centered at the unique vertex of $G_{0}$.

Let $u \in B_{p}^{0}(Z)$ with $u(x) \neq u(y)$. Changing $u$ to $a u+b(a, b \in \mathbb{R})$ if necessary, we can assume that there exist open subsets $U, V$ of $Z$ with $x \in U, y \in V$ and $u \leq 0$ on $U, u \geq 1$ on $V$. Pick a function $f: G^{0} \rightarrow \mathbb{R}$ such that $d f \in \ell_{p}\left(G^{1}\right)$ and $f_{\infty}=u$. Choosing $f$ properly one can ensure that for $k$ large enough and for every $v \in G_{k}^{0}$ the following holds:

$$
f(v) \leq 1 / 3 \text { if } v \cap U \neq \emptyset, \quad f(v) \geq 2 / 3 \text { if } v \cap V \neq \emptyset,
$$

(see [BP03], proof of Théorème 3.4). Let $\rho_{k}: G_{k}^{0} \rightarrow \mathbb{R}_{+}$be defined by

$$
\rho_{k}(v)=3 \max _{(v, w) \in G_{k}^{1}}|f(v)-f(w)|
$$

Obviously it is an $\mathcal{F}(U, V)$-admissible function. In addition its $p$-mass satisfies

$$
M_{p}\left(\rho_{k}\right) \leq 3^{p} \sum_{a \in G_{k}^{1}}|d f(a)|^{p} .
$$

For $d f \in \ell_{p}\left(G^{1}\right)$ the right-hand side term tends to 0 when $k$ tends to $+\infty$.

Combining several previous results we now collect some applications to hyperbolic Coxeter groups. 
Corollary 10.3. Let $\Gamma$ be a hyperbolic Coxeter group. Then each coset of the $\ell_{p^{-}}$ equivalence relation on $\partial \Gamma$ is either a point or a connected parabolic limit set.

Proof. It is a straightforward consequence of Proposition 10.1 and Corollary 5.8.

Corollary 10.4. Let $\Gamma$ be a hyperbolic Coxeter group with connected boundary. Let $p \geq 1$ and suppose that $\sim_{p}$ admits a coset different from a point and the whole $\partial \Gamma$. Then $\sim_{p}$ admits a coset $F$ with $\operatorname{Confdim}(F)=\operatorname{Confdim}(\partial \Gamma)$.

Proof. Let $p$ be as in the statement. At first we notice that Corollary 10.5 below implies that $p \leq Q_{M}$. Let $\mathscr{L}$ be the collection of the cosets of $\sim_{p}$ which are different from a point. From the above corollary its elements are connected proper parabolic limit sets. Obviously the $\Gamma$-invariance and separation hypotheses of Corollary 6.4 are satisfied. In addition, for every $r>0$, Proposition 10.2 shows that $\operatorname{Mod}_{p}\left(\mathscr{F}_{r}(\mathscr{L}), G_{k}\right)$ tends to 0 when $k$ tends to $+\infty$. The same holds for every $q \geq p$ by monotonicity of the function $q \mapsto \operatorname{Mod}_{q}$. Thus it holds for $Q_{M}$. Assume by contradiction that $\operatorname{Confdim}(\mathscr{L})<\operatorname{Confdim}(\partial \Gamma)$. Since $\operatorname{Confdim}(\partial \Gamma)=Q_{M}$ (see assertion 2 of the remark at the end of Section 3), one can apply Corollary 6.4 with exponent $Q_{M}$. One obtains that $\operatorname{Mod}_{Q_{M}}\left(\mathcal{F}_{0}, G_{k}\right)$ tends to 0 when $k$ tends to $+\infty$, contradicting Corollary 3.7 (2).

Finally we return to general approximately self-similar metric spaces and to the combinatorial Loewner property. The second part of the following corollary is the combinatorial analog of Théorème 0.3 in [BP03].

Corollary 10.5. Let $Z$ be an approximately self-similar metric space. Assume $Z$ is connected and let $p \geq 1$. Then $p>Q_{M}$ if and only if $\left(Z / \sim_{p}\right)=Z$. If in addition $Z$ satisfies the $C L P$, then for $1 \leq p \leq Q_{M}$ the quotient $Z / \sim_{p}$ is a singleton.

Proof. The second part of the statement follows from Proposition 10.2 and from the monotonicity of $p \mapsto \operatorname{Mod}_{p}$. To establish the "only if" part of the first, one invokes that $Q_{M}$ is equal to the Ahlfors regular conformal dimension of $Z$ (see Remark 2 at the end of Section 3), and the fact that $B_{p}^{0}(Z)$ separates the points of $Z$ for $p$ strictly larger than the Ahlfors-regular conformal dimension [BP03]. Conversely if $\left(Z / \sim_{p}\right)=Z$, then Proposition 10.2 implies that $\operatorname{Mod}_{p}\left(\mathcal{F}_{0}, G_{k}\right)$ tends to 0 when $k$ tends to $+\infty$. With Corollary 3.7 (2) we get that $p>Q_{M}$.

Remarks and questions. Corollary 10.5 may be used to produce examples of spaces for which the CLP fails. Indeed suppose that for a given hyperbolic group $\Gamma$, the family of quotient spaces $\partial \Gamma / \sim_{p},(p \in[1,+\infty))$, contains intermediate states between the singleton and the whole $\partial \Gamma$. Then, according to Corollary 10.5, $\partial \Gamma$ does not admit the CLP. 
Currently all known examples of hyperbolic groups for which the CLP fails are of two types. Either their boundaries admit local cut points - in which case the CLP fails "trivially" (see Proposition 2.5.2) - or they decompose as $\Gamma=A \star_{C} B$ and there exists $p \in\left[1, Q_{M}\right]$ with

$$
x \sim_{p} y \Longleftrightarrow x=y \text { or } x, y \in g \partial B \text { for some } g \in \Gamma .
$$

Examples of the second type, including some Coxeter groups, are given in [Bou04]. In the Coxeter group case, Corollary 10.4 shows that $\operatorname{Confdim}(\partial B)=\operatorname{Confdim}(\partial \Gamma)$.

It would be desirable to have a better understanding of the relations between the combinatorial modulus and the $\ell_{p}$-cohomology.

\section{References}

[Bar03] M. T. Barlow, Heat kernels and sets with fractal structure. In Heat kernels and analysis on manifolds, graphs, and metric spaces (Paris, 2002), Contemp. Math. 338, Amer. Math. Soc., Providence, RI, 2003, 11-40. Zbl 1056.60072 MR 2039950

[Ben06] Y. Benoist, Convexes hyperboliques et quasiisométries. Geom. Dedicata 122 (2006), 109-134. Zbl 1122.20020 MR 2295544

[BM91] M. Bestvina and G. Mess, The boundary of negatively curved groups. J. Amer. Math. Soc. 4 (1991), 469-481. Zbl 0767.20014 MR 1096169

[Big74] N. Biggs, Algebraic graph theory. Cambridge Tracts in Math. 67, Cambridge University Press, London 1974. Zbl 0284.05101 MR 0347649

[Bon06] M. Bonk, Quasiconformal geometry of fractals. In Proc. Internat. Congr. Mathematicians, Vol. II, Eur. Math. Soc., Zürich 2006, 1349-1373. Zbl 1102.30016 MR 2275649

[BK02] M. Bonk and B. Kleiner, Quasisymmetric parametrizations of two-dimensional metric spheres. Invent. Math. 150 (2002), 127-183. Zbl 1037.53023 MR 1930885

[BK05a] M. Bonk and B. Kleiner, Conformal dimension and Gromov hyperbolic groups with 2-sphere boundary. Geom. Topol. 9 (2005), 219-246. Zbl 1087.20033 MR 2116315

[BK05b] M. Bonk and B. Kleiner, Quasi-hyperbolic planes in hyperbolic groups. Proc. Amer. Math. Soc. 133 (2005), 2491-2494. Zbl 1123.20036 MR 2146190

[BM] M. Bonk and D. Meyer, Expanding Thurston maps. Preprint 2010. arXiv:1009.3647

[Bou04] M. Bourdon, Cohomologie $l_{p}$ et produits amalgamés. Geom. Dedicata 107 (2004), 85-98. Zbl 1124.20025 MR 2110755

[BP00] M. Bourdon and H. Pajot, Rigidity of quasi-isometries for some hyperbolic buildings. Comment. Math. Helv. 75 (2000), 701-736. Zbl 0976.30011 MR 1789183

[BP02] M. Bourdon and H. Pajot, Quasi-conformal geometry and hyperbolic geometry. In Rigidity in dynamics and geometry (Cambridge, 2000), Springer-Verlag, Berlin 2002, 1-17. Zbl 1002.30012 MR 1919393 
[BP03] M. Bourdon and H. Pajot, Cohomologie $l_{p}$ et espaces de Besov. J. Reine Angew. Math. 558 (2003), 85-108. Zbl 1044.20026 MR 1979183

[Bow99] B. H. Bowditch, Convergence groups and configuration spaces. In Geometric group theory down under (Canberra, 1996), de Gruyter, Berlin 1999, 23-54. Zbl 0952.20032 MR 1714838

[BH99] M. R. Bridson and A. Haefliger, Metric spaces of non-positive curvature. Grundlehren Math. Wiss. 319, Springer-Verlag, Berlin 1999. Zbl 0988.53001 MR 1744486

[Can94] J. W. Cannon, The combinatorial Riemann mapping theorem. Acta Math. 173 (1994), 155-234. Zbl 0832.30012 MR 1301392

[CFP99] J. W. Cannon, W. J. Floyd, and W. R. Parry, Sufficiently rich families of planar rings. Ann. Acad. Sci. Fenn. Math. 24 (1999), 265-304. Zbl 0939.20048 MR 1724092

[CS98] J. W. Cannon and E. L. Swenson, Recognizing constant curvature discrete groups in dimension 3. Trans. Amer. Math. Soc. 350 (1998), 809-849. Zbl 0910.20024 MR 1458317

[Car] M. Carrasco Piaggio, Jauge conforme des espaces métriques compacts. PhD thesis, Aix-Marseille Université, Marseille 2011. http://tel.archives-ouvertes.fr/tel-00645284

[Che99] J. Cheeger, Differentiability of Lipschitz functions on metric measure spaces. Geom. Funct. Anal. 9 (1999), 428-517. Zbl 0942.58018 MR 1708448

[Dav08] M. W. Davis, The geometry and topology of Coxeter groups. London Math. Soc. Monogr. Ser. 32, Princeton University Press, Princeton, NJ, 2008. Zbl 1142.20020 MR 2360474 (2008k:20091)

[Ele97] G. Elek, The $l_{p}$-cohomology and the conformal dimension of hyperbolic cones. Geom. Dedicata 68 (1997), 263-279. Zbl 0899.53035 MR 1486435

[Ess96] F. Esselmann, The classification of compact hyperbolic Coxeter $d$-polytopes with $d+2$ facets. Comment. Math. Helv. 71 (1996), 229-242. Zbl 0856.51016 MR 1396674

[Gro87] M. Gromov, Hyperbolic groups. In Essays in group theory, Math. Sci. Res. Inst. Publ. 8, Springer-Verlag, New York 1987, 75-263. Zbl 0634.20015 MR 0919829

[Gro93] M. Gromov, Geometric group theory (Sussex, 1991), vol. 2: Asymptotic invariants of infinite groups. London Math. Soc. Lecture Note Ser. 182, Cambridge University Press, Cambridge 1993. Zbl 0841.20039 MR 1253544

[GP91] M. Gromov and P. Pansu, Rigidity of lattices: an introduction. In Geometric topology: recent developments (Montecatini Terme, 1990), Lecture Notes in Math. 1504, Springer-Verlag, Berlin 1991, 39-137. Zbl 0786.22015 MR 1168043

[Hag98] F. Haglund, Réseaux de Coxeter-Davis et commensurateurs. Ann. Inst. Fourier (Grenoble) 48 (1998), 649-666. Zbl 0899.20019 MR 1644077

[Hai09] P. Haïssinsky, Empilements de cercles et modules combinatoires. Ann. Inst. Fourier (Grenoble) 59 (2009), 2175-2222. Zbl 1189.30080 MR 2640918

[HP09] P. Haïssinsky and K. M. Pilgrim, Coarse expanding conformal dynamics. Astérisque 325, Soc. Math. France, Paris 2009. Zbl 1206.37002 MR 2662902 
[HP] P. Haïssinsky and K. M. Pilgrim, Minimal Ahlfors regular conformal dimension of coarse conformal dynamics on the sphere. Preprint 2011. arXiv:1103.4019

[Hei01] J. Heinonen, Lectures on analysis on metric spaces. Universitext, Springer-Verlag, New York 2001. Zbl 0985.46008 MR 1800917

[HK98] J. Heinonen and P. Koskela, Quasiconformal maps in metric spaces with controlled geometry. Acta Math. 181 (1998), 1-61. Zbl 0915.30018 MR 1654771

[HKST01] J. Heinonen, P. Koskela, N. Shanmugalingam, and J. T. Tyson, Sobolev classes of Banach space-valued functions and quasiconformal mappings. J. Anal. Math. 85 (2001), 87-139. Zbl 1013.46023 MR 1869604

[Kap74] I. M. Kaplinskaya, Discrete groups generated by reflections in the faces of symplicial prisms in Lobachevskian spaces. Mat. Zametki 15 (1974), 159-164; English transl. Math. Notes 15 (1974), 88-91. Zbl 0288.50014 MR 0360858

[KB02] I. Kapovich and N. Benakli, Boundaries of hyperbolic groups. In Combinatorial and geometric group theory (New York, 2000/Hoboken, NJ, 2001), Contemp. Math. 296, Amer. Math. Soc., Providence, RI, 2002, 39-93. Zbl 1044.20028 MR 1921706

[KaK00] M. Kapovich and B. Kleiner, Hyperbolic groups with low-dimensional boundary. Ann. Sci. École Norm. Sup. (4) 33 (2000), 647-669. Zbl 0989.20031 MR 1834498

$[\mathrm{KeK}] \quad$ S. Keith and B. Kleiner, in preparation.

[KZ08] S. Keith and X. Zhong, The Poincaré inequality is an open ended condition. Ann. of Math. (2) 167 (2008), 575-599. Zbl 1180.46025 MR 2415381

[Kle06] B. Kleiner, The asymptotic geometry of negatively curved spaces: uniformization, geometrization and rigidity. In Proc. Internat. Congr. Math., Vol. II, Eur. Math. Soc., Zürich 2006, 743-768. Zbl 1108.30014 MR 2275621

[Mac10] J. M. Mackay, Spaces and groups with conformal dimension greater than one. Duke Math. J. 153 (2010), 211-227. Zbl 05731758 MR 2667133

[MT10] J. M. Mackay and J. T. Tyson, Conformal dimension. Univ. Lecture Ser. 54, Amer. Math. Soc., Providence, RI, 2010. Zbl 1201.30002 MR 2662522

[Mat95] P. Mattila, Geometry of sets and measures in Euclidean spaces. Cambridge Stud. Adv. Math. 44, Cambridge University Press, Cambridge 1995. Zbl 0819.28004 MR 1333890

[Mey10] D. Meyer, Snowballs are quasiballs. Trans. Amer. Math. Soc. 362 (2010), 1247-1300. Zbl 1190.30018 MR 2563729

[Mun75] J. R. Munkres, Topology: a first course. Prentice-Hall Inc., Englewood Cliffs, N.J., 1975. Zbl 0306.54001 MR 0464128

[Pan89a] P. Pansu, Cohomologie $L^{p}$ des variétés à courbure négative, cas du degré 1. Rend. Sem. Mat. Univ. Politec. Torino, Torino Fasc. Spec., 1989, 95-120. Zbl 0723.53023 MR 1086210

[Pan89b] P. Pansu, Dimension conforme et sphère à l'infini des variétés à courbure négative. Ann. Acad. Sci. Fenn. Ser. A I Math. 14 (1989), 177-212. Zbl 0722.53028 MR 1024425 
[Ron89] M. Ronan, Lectures on buildings. Perspect. Math. 7, Academic Press, Boston 1989. Zbl 0694.51001 MR 1005533

[Str83] R. S. Strichartz, Analysis of the Laplacian on the complete Riemannian manifold. J. Funct. Anal. 52 (1983), 48-79. Zbl 0515.58037 MR 705991

[Sul81] D. Sullivan, On the ergodic theory at infinity of an arbitrary discrete group of hyperbolic motions. In Riemann surfaces and related topics: Proceedings of the 1978 Stony Brook Conference, Ann. of Math. Stud. 97, Princeton Univ. Press, Princeton, N.J., 1981, 465-496. Zbl 0567.58015 MR 624833

[Tuk86] P. Tukia, On quasiconformal groups. J. Analyse Math. 46 (1986), 318-346. Zbl 0603.30026 MR 861709

[Tys98] J. Tyson, Quasiconformality and quasisymmetry in metric measure spaces. Ann. Acad. Sci. Fenn. Math. 23 (1998), 525-548. Zbl 0910.30022 MR 1642158

[V̈̈5] J. Väisälä, Quasimöbius maps. J. Analyse Math. 44 (1984), 218-234. Zbl 0593.30022 MR 801295

[Vin85] È. B. Vinberg, Hyperbolic reflection groups. Uspekhi Mat. Nauk 40 (1985), No. 1, 29-66; English transl. Russian Math. Surveys 40 (1985), No. 1, 31-75. Zbl 0579.51015 MR 783604

Received February 10, 2010; revised July 4, 2011

M. Bourdon, Département de Mathématiques, Université Lille 1, Bat. M2, 59655 Villeneuve d'Ascq, France

E-mail: bourdon@math.univ-lille1.fr

B. Kleiner, Courant Institute, New York University, 251 Mercer Street, New York, NY 10012-1185, U.S.A.

E-mail: bkleiner@courant.nyu.edu 